\section{Pacific Northwest}

National Laboratory

Operated by Battelle for the

U.S. Department of Energy

\title{
Metals Verification Study for Sinclair and Dyes Inlets, Washington
}

\author{
N.P. Kohn \\ M. C. Miller \\ J. M. Brandenberger \\ Battelle Marine Sciences Laboratory \\ Sequim, Washington \\ R. K. Johnston \\ Space and Naval Warfare Systems Center \\ Bremerton, Washington
}

September 2004

Prepared for the

Puget Sound Naval Shipyard and

Intermediate Maintenance Facility

Project ENVVEST

Bremerton, Washington

under Contract DE-AC06-76RLO 1830

Pacific Northwest National Laboratory

Richland, Washington 


\title{
DISCLAIMER
}

This report was prepared as an account of work sponsored by an agency of the United States Government. Neither the United States Government nor any agency thereof, nor Battelle Memorial Institute, nor any of their employees, makes any warranty, express or implied, or assumes any legal liability or responsibility for the accuracy, completeness, or usefulness of any information, apparatus, product, or process disclosed, or represents that its use would not infringe privately owned rights. Reference herein to any specific commercial product, process, or service by trade name, trademark, manufacturer, or otherwise does not necessarily constitute or imply its endorsement, recommendation, or favoring by the United States Government or any agency thereof, or Battelle Memorial Institute. The views and opinions of authors expressed herein do not necessarily state or reflect those of the United States Government or any agency thereof.

\author{
PACIFIC NORTHWEST NATIONAL LABORATORY \\ operated by \\ BATTELLE \\ for the \\ UNITED STATES DEPARTMENT OF ENERGY \\ under Contract DE-AC06-76RL01830
}

This document was printed on recycled paper. 
PNNL-14872

METALS VERIFICATION STUDY FOR SINCLAIR

AND DYES INLETS, WASHINGTON

N. P. Kohn

M. C. Miller

J. M. Brandenberger

Battelle Marine Sciences Laboratory

Sequim, Washington

R. K. Johnston

Space and Naval Warfare Systems Center

Bremerton, Washington

September 2004

Prepared for

Puget Sound Naval Shipyard and

Intermediate Maintenance Facility

Project ENVVEST

Bremerton, Washington

Under Contract No. DE-AC06-76RLO 1830

Pacific Northwest National Laboratory

Richland, Washington 99352 


\section{SUMMARY}

Sinclair and Dyes Inlets near Bremerton, Washington, are on the State of Washington’s 1998 303(d) list of impaired waters because of fecal coliform contamination in marine water, metals in sediment and fish tissue, and organics in sediment and fish tissue. Because significant cleanup and source control activities have been conducted in the inlets since the data supporting the 1998 303(d) listings were collected, this Metals Verification Study was conducted to address the 303(d) segments that are listed for metal contaminants in marine sediment. The study was designed to obtain representative data on current sediment metals concentrations throughout Sinclair Inlet, Dyes Inlet, Port Orchard Passage, and Rich Passage, with stations spatially distributed to support 303(d) listing updates and also watershed-level water quality and contaminant transport modeling efforts. A total of 160 surface sediment samples from Sinclair Inlet, Dyes Inlet, Port Orchard Passage, and Rich Passage were screened for copper, lead, and zinc using X-ray fluorescence (XRF). Of these 160 samples, 40 samples (25\%) were selected for confirmatory metals analysis by inductively coupled plasma mass spectroscopy (ICP-MS) for cadmium, silver, and arsenic in addition to copper, lead, and zinc. Regression relationships between the ICP-MS and XRF datasets were developed to estimate copper, lead, and zinc concentrations in all samples. The XRF results for copper, lead, and zinc correlated well with ICP-MS results, and predicted concentrations were calculated for all samples. The results of the Metals Verification Study show that sediment quality in Sinclair Inlet has improved markedly since implementation of cleanup and source control actions, and that the distribution of residual contaminants is limited to nearshore areas already within the actively managed Puget Sound Naval Shipyard Superfund Site where further source control actions and monitoring are underway. Outside of the immediate vicinity of the PSNS Superfund site in Sinclair Inlet, the target metals concentrations met state sediment quality standards. 


\section{CONTENTS}

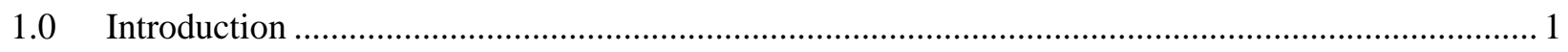

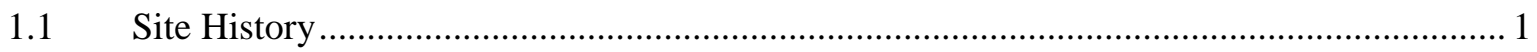

1.2 Metals Verification Study Background and Objectives ................................................ 6

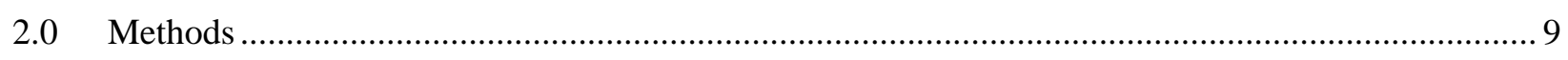

2.1 Metals Verification Study Design .............................................................................. 9

2.1.1 Sampling Design for Sinclair Inlet, OU B Marine................................................... 9

2.1.2 Dyes Inlet, Ostrich Bay, Port Orchard Passage, and Rich Passage........................... 11

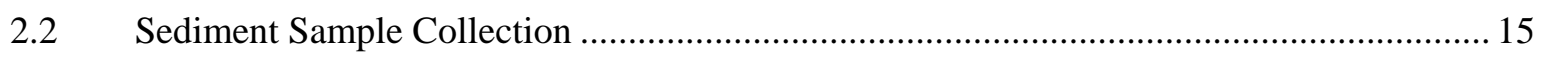

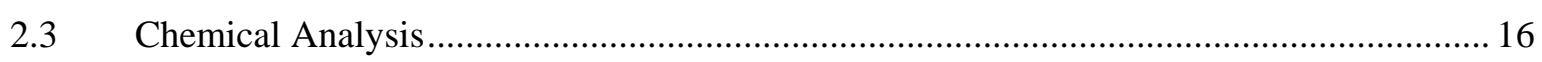

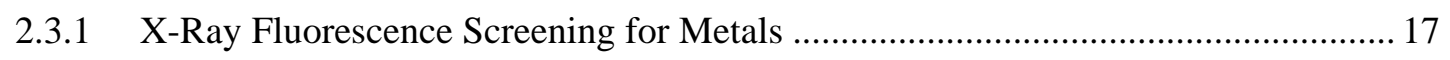

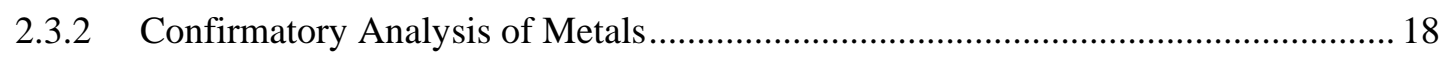

2.3.3 Ancillary Sediment Analyses Outside of Metals Verification Study........................ 19

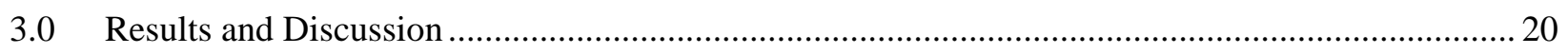

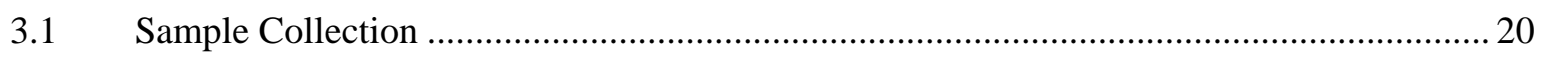

3.1.1 Dyes Inlet, Ostrich Bay, Port Orchard Passage, and Rich Passage........................... 20

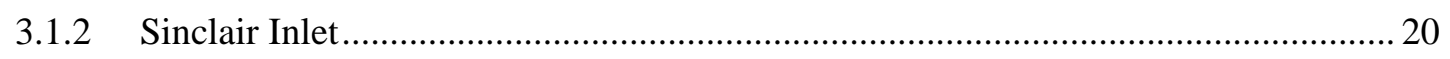

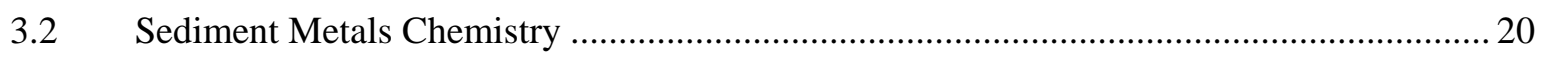

3.3 Correlation of Screening and Quantitative Results ..................................................... 38

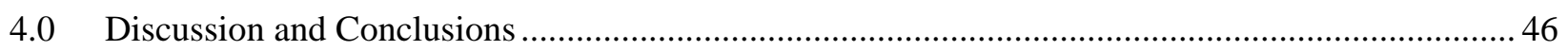

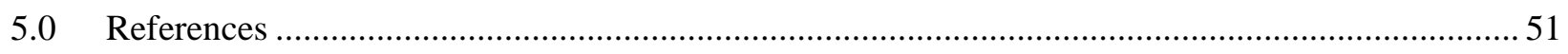

APPENDIX A X-Ray Fluorescence Laboratory Report

APPENDIX B Inductively-Coupled Plasma-Mass Spectroscopy Data Report 


\section{FIGURES}

Figure 1. Sinclair and Dyes Inlet Study Area Showing Watershed Subbasins ........................................ 2

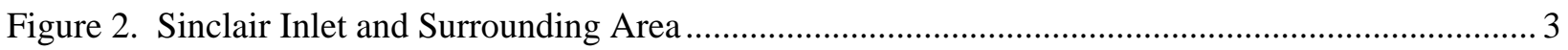

Figure 3. Dyes Inlet, Ostrich Bay, Port Washington Narrows, and Surrounding Area ............................. 4

Figure 4. Storm Water Outfalls and Combined Sewer Overflows in the City of Bremerton ...................... 5

Figure 5. Buildings, Catch Basins, and Major Stormwater Outfalls Within the Bremerton

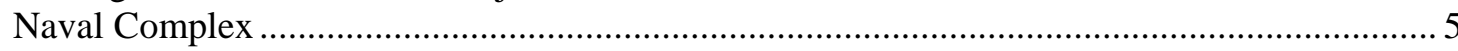

Figure 6. Schematic Drawing of the process to assess 303(d) metals listings in Sinclair and

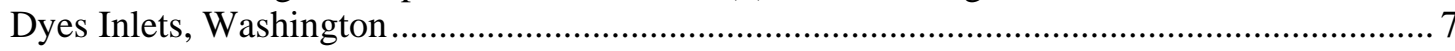

Figure 7. 500-ft monitoring grids within the OU B Marine boundary (OUBM), with 303(d) segment overlay (blue)

Figure 8. 1500-ft monitoring grids outside of the OU B Marine boundary (OOUB), with 303(d) segment overlay (blue).

Figure 9. Planned Metals Verification Study (black dots and crosses) and model initialization (green triangles) stations in Dyes Inlet, Port Washington Narrows, and Port Orchard Passage

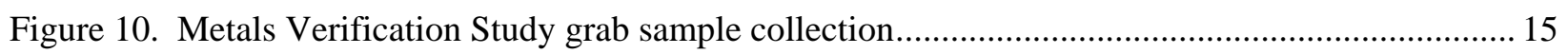

Figure 11. Linear correlation of XRF and ICP-MS Cu, Pb, and Zn measurements ............................... 17

Figure 12. Metals Verification Study stations sampled in Dyes Inlet, including Ostrich Bay ..................21

Figure 13. Metals Verification Study stations sampled in Port Orchard Passage and Rich Passage.

Figure 14. OU B Marine monitoring stations in 500-ft grid within OU B Marine boundary (OUBM).

Figure 15. OU B Marine monitoring stations in 1500-ft grid outside OU B Marine boundary (OOUB)

Figure 16. Linear correlations of XRF and ICP-MS measurements for $\mathrm{Cu}$ (top), $\mathrm{Pb}$ (middle), Zn (bottom), ENVVEST Metals Verification Study....

Figure 17. Definitive sediment $\mathrm{Cu}$ (top), $\mathrm{Pb}$ (middle), and Zn (bottom) ENVVEST Metals Verification Study.

Figure 18. Locations where definitive concentrations exceed SQS or MCUL in entire Metals Verification Study area

Figure 19. Locations where definitive concentrations exceed SQS or MCUL in Sinclair Inlet 


\section{TABLES}

Table 1. Metals Verification Study Design Summary for Sinclair and Dyes Inlets ................................ 10

Table 2. XRF Screening and ICP-MS Detection Limits Compared with State Sediment Management Standards for ENVVEST Metals of Concern ................................................. 18

Table 3. Metals Verification Study Sample Collection Information for Dyes Inlet, Port Orchard Passage, and Rich Passage.

Table 4. OU B Marine Monitoring Samples Received for Metals Verification Study ............................ 29

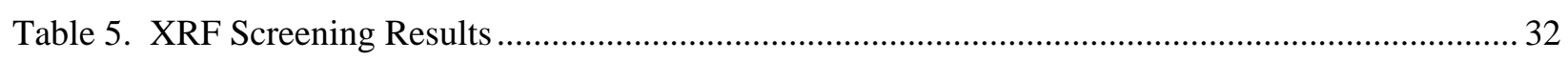

Table 6. Samples Selected for Confirmatory Metals Analysis by ICP-MS .......................................... 37

Table 7. Metals Verification Study Sample Analysis Summary .......................................................... 37

Table 8. Confirmatory Metals Analysis Results, Metals Verification Study........................................... 39

Table 9. Definitive Sediment Metals Concentrations, Metals Verification Study ................................... 42 


\subsection{INTRODUCTION}

Sinclair and Dyes Inlets near Bremerton, Washington, are on the State of Washington's 1998 303(d) list of impaired waters because of fecal coliform contamination in marine water, metals in sediment and fish tissue, and organics in sediment and fish tissue (Ecology 2003) Sources of pollution include industry such as Puget Sound Naval Shipyard (PSNS) and Intermediate Maintenance Facility (IMF), publicly owned treatment works (POTW), combined sewer overflows, stormwater outfalls, marinas and private shipworks, and nonpoint source stormwater runoff. A partnership between the Navy, U.S. Environmental Protection Agency (EPA), state agencies, local governments, and the Suquamish Tribe was established to address water quality issues related to the PSNS and IMF facilities. This partnership, called Project ENVVEST (Environmental Investment), set a priority to address the development of total maximum daily loads (TMDLs) for the Sinclair and Dyes Inlets system. The first TMDL study initiated by Project ENVVEST was to support development of a fecal coliform TMDL for the inlets; the fecal coliform technical study is currently being completed. The second TMDL study being considered for execution is the development of a TMDL study plan for trace metals that exceed Washington State sediment quality standards (SQS) or minimum cleanup levels (MCUL). Future TMDL studies are planned that will address toxic organics in the sediment, mercury in sediment, nutrients and dissolved oxygen in the water column, and toxic chemicals in fish tissue.

The present Metals Verification Study for Sinclair and Dyes Inlets addresses the second ENVVEST priority: the 303(d) segments that are listed for metal contaminants in marine sediment. Most of the data supporting the 303(d) listings were collected in 1998 or earlier. Since those data were collected, significant cleanup and source control activities have been conducted in the inlets. The Metals Verification Study was conducted to provide recent metal concentration data for sediment throughout the study area and especially in the more contaminated areas where cleanup actions were implemented, to determine whether metals remain a source of sediment quality impairment.

\subsection{Site History}

The Sinclair and Dyes Inlets watershed (Figure 1) is home to the PSNS, located on Sinclair Inlet, an arm on the west side of Puget Sound. Sinclair Inlet is open to Puget Sound through the Port Orchard Passage, which extends northward along the west side of Bainbridge Island and through Rich Passage, which exits to the southeast (Figure 2). Immediately to the east of the PSNS, the Port Washington Narrows enters into Dyes Inlet (Figure 3). Sediment in both Sinclair and Dyes Inlets is the subject of this investigation. 


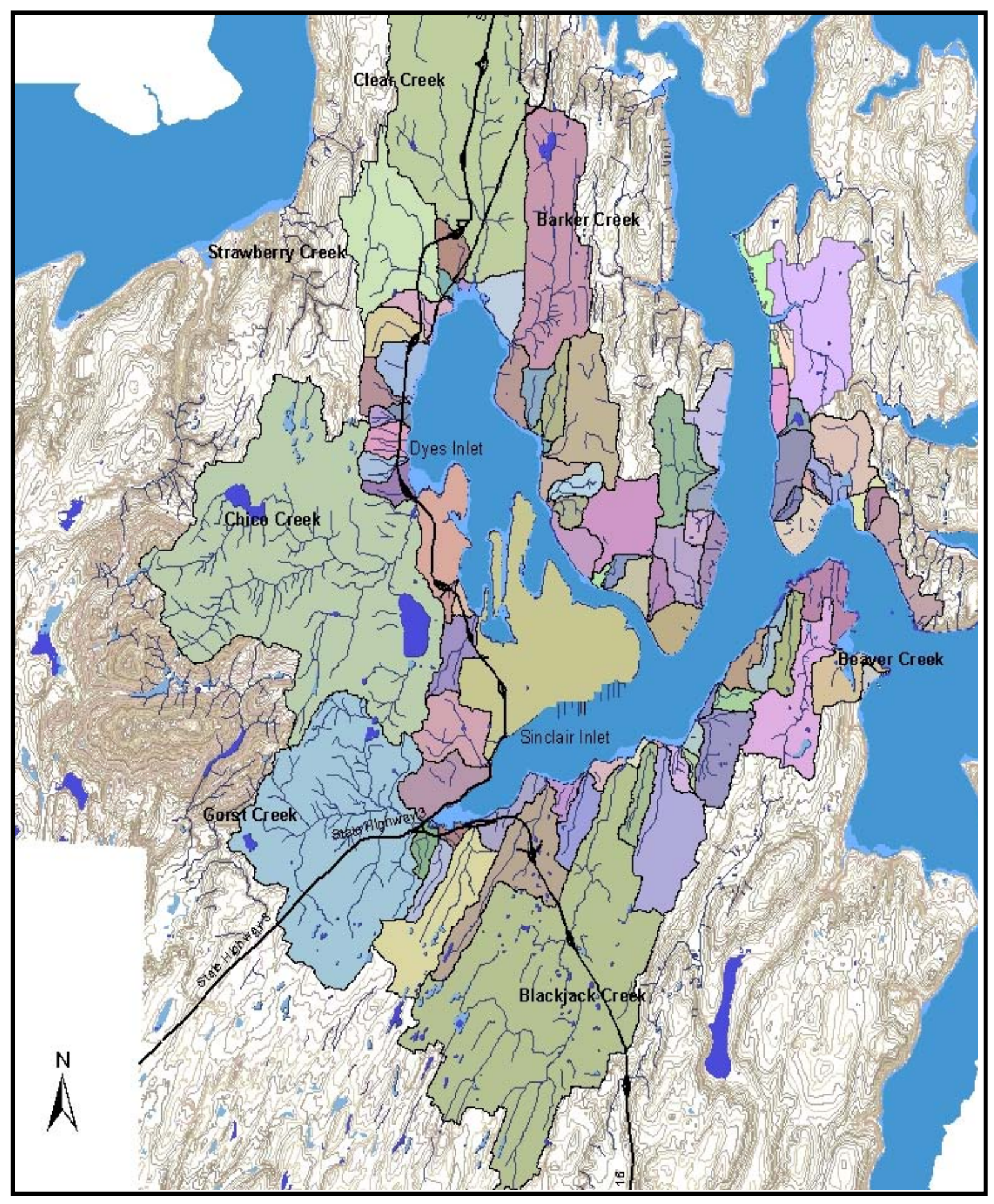

Figure 1. Sinclair and Dyes Inlet Study Area Showing Watershed Subbasins 


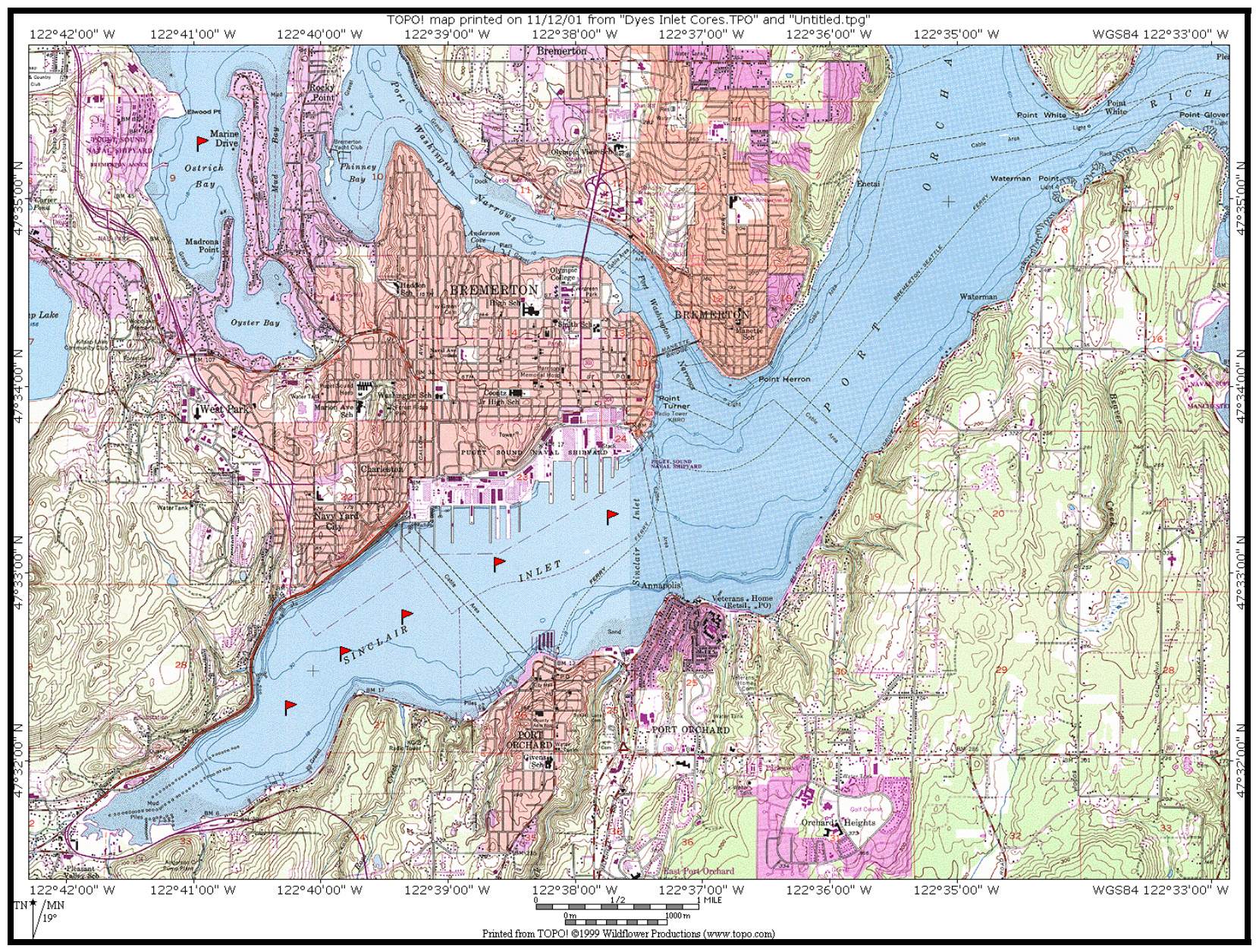

Figure 2. Sinclair Inlet and Surrounding Area

Previous studies indicated that marine sediment in Sinclair Inlet has been contaminated with heavy metals, polychlorinated biphenyls (PCBs), and polynuclear aromatic hydrocarbons (PAHs).

Contaminants released within the inlets as well as contaminants transported into the inlets from the greater Puget Sound will tend to accumulate in depositional areas (muddy sediment deposits) located within Sinclair and Dyes Inlets, Port Orchard Passage and Rich Passage. Historical geographic distributions have shown that PSNS was a significant source of the contamination (Katz et al., 1999; URS 2002a). Other sources include municipal wastewater, marinas, industrial and agricultural effluents, nonpoint source runoff, and atmospheric deposition. Storm water outfalls and combined sewer overflows (CSOs) discharge to Sinclair and Dyes Inlets and the Port Washington Narrows; those associated with the City of Bremerton and PSNS are shown in Figures 4 and 5. 


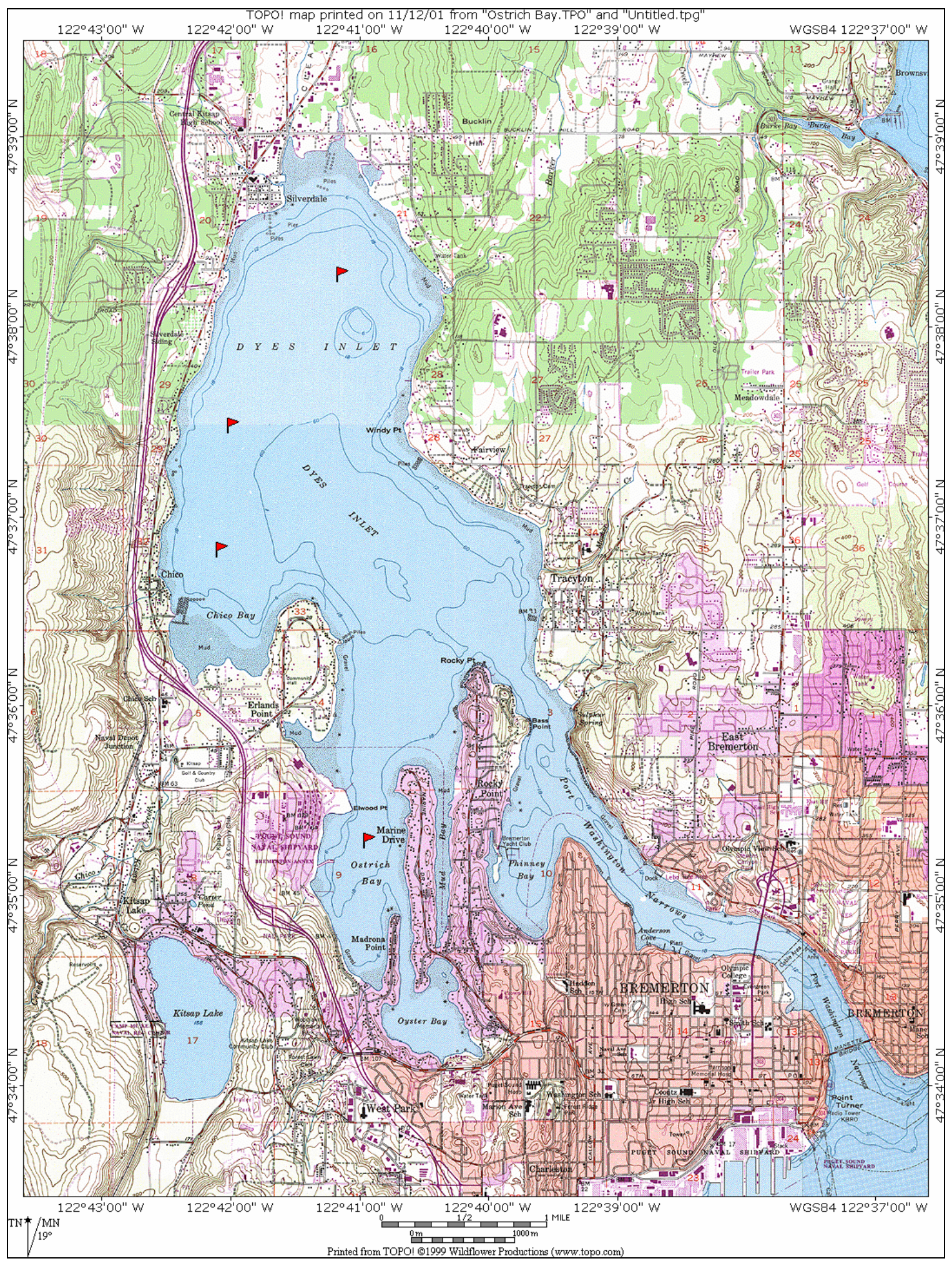

Figure 3. Dyes Inlet, Ostrich Bay, Port Washington Narrows, and Surrounding Area 


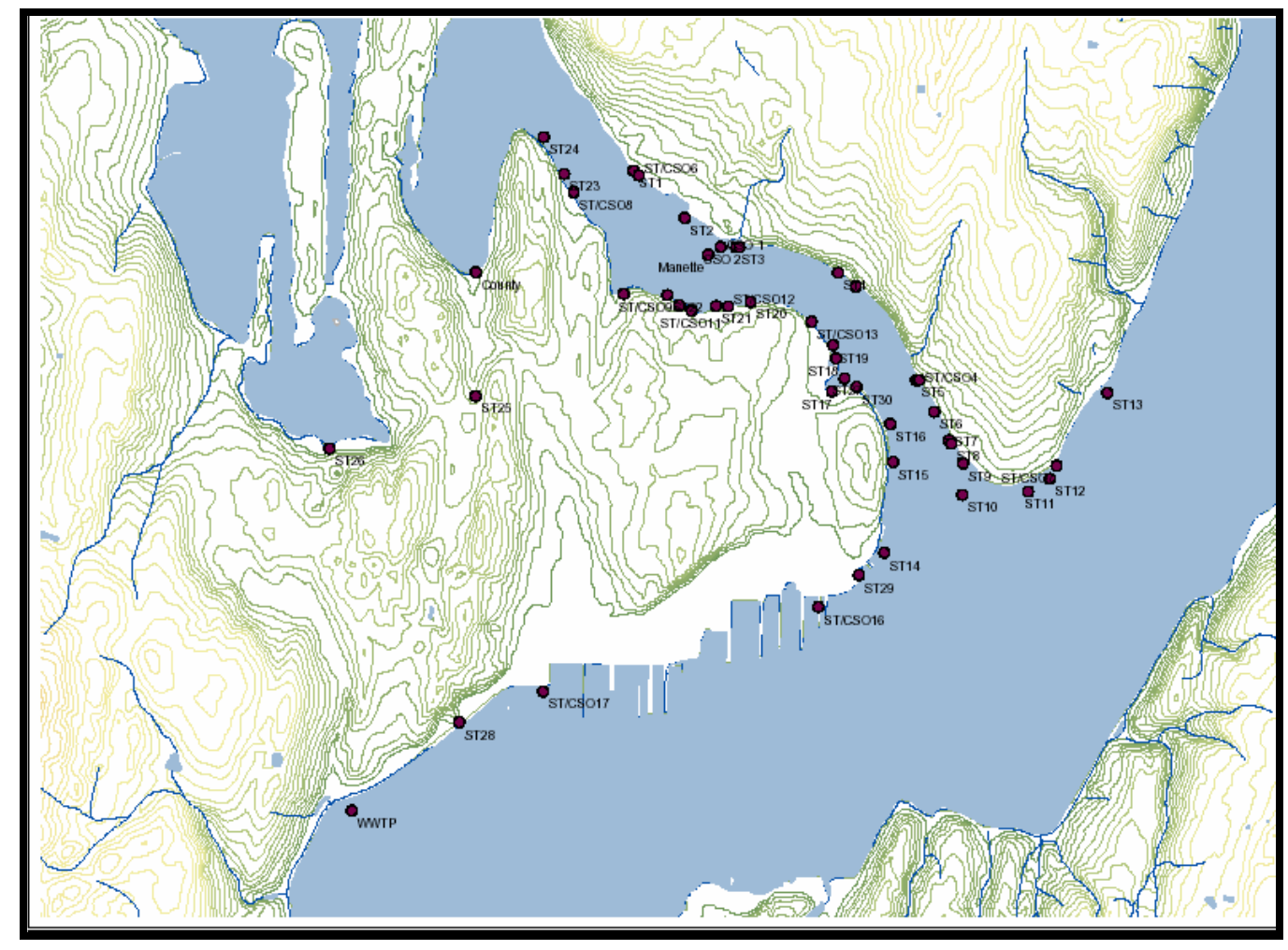

Figure 4. Storm Water Outfalls and Combined Sewer Overflows in the City of Bremerton

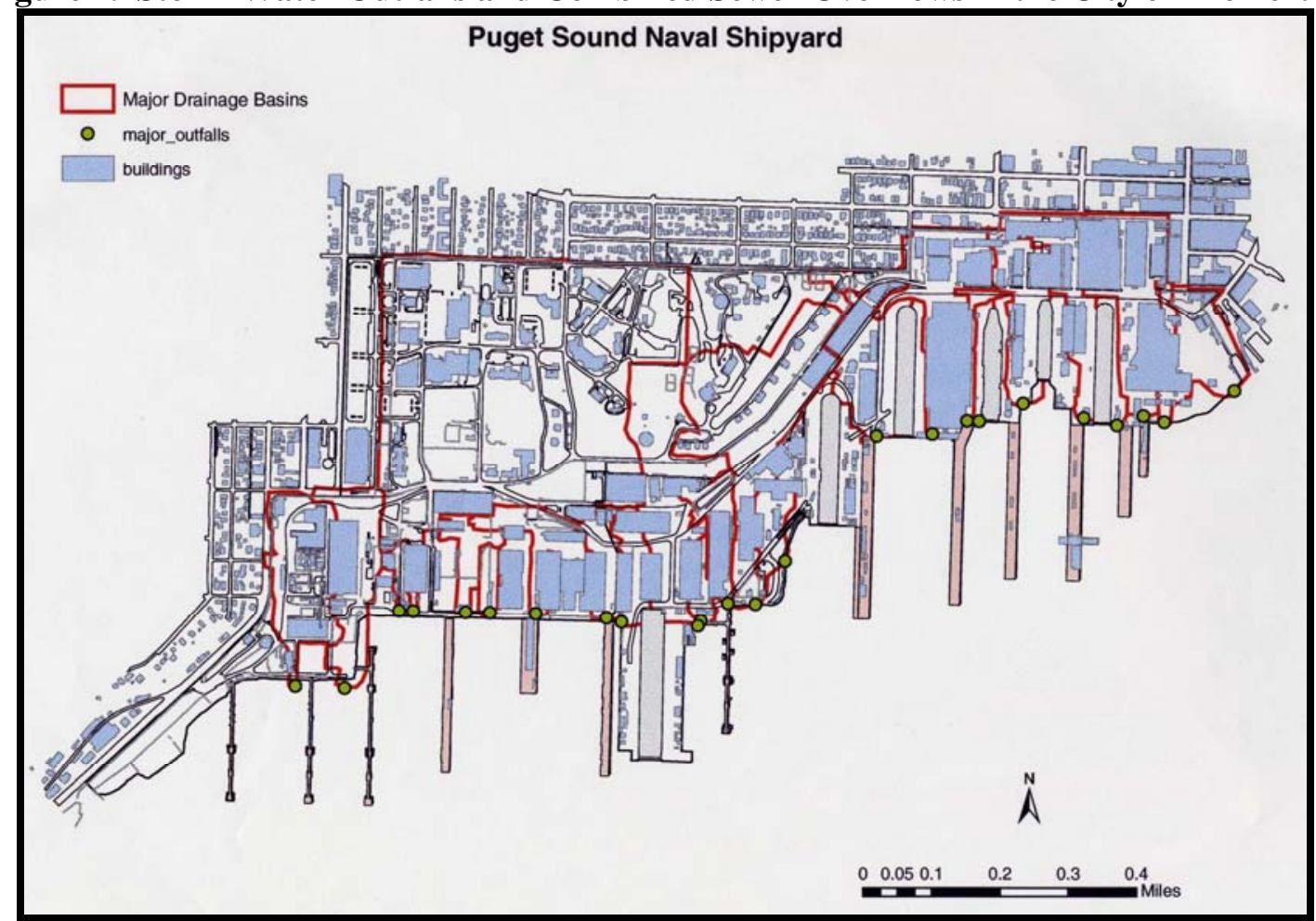

Figure 5. Buildings, Catch Basins, and Major Stormwater Outfalls Within the Bremerton Naval Complex 
The 1998 303(d) list included arsenic (As), cadmium (Cd), copper (Cu), lead (Pb), mercury (Hg), and zinc (Zn) in sediments of Sinclair Inlet and Cd, Hg, and silver (Ag) in the sediments of Dyes Inlet. Currently, two segments in Sinclair Inlet are listed for these metals of concern (47122F6F3 and 47122F6F4) and one in Dyes Inlet (47122F618). The 1998 303(d) listings were largely based on sediment data collected as part of remedial investigations and navigational dredging programs conducted for the Bremerton Naval Complex in Sinclair Inlet (U.S. Navy 2000a, 2003) and Jackson Park in Dyes Inlet (U.S. Navy 2000b). Most of the locations where existing sediment metals data exceed SQS are located immediately offshore of PSNS; the offshore sediment is part of a Superfund site and managed under the federal facilities Installation Restoration (IR) cleanup program by the Navy's Engineering Field Activity Northwest (EFANW). The marine sediment is designated as Operable Unit (OU) B Marine. Significant contaminated sediment removal and capping activities were performed in OU B Marine in 2000-2001. Post-remediation monitoring was planned, with the first round of monitoring sampling occurring in fall 2003.

\subsection{Metals Verification Study Background and Objectives}

During development of the metals TMDL study plan, the Project ENVVEST Technical Team conducted a review of the available sediment and water quality information for Sinclair and Dyes Inlets (Diefenderfer et al. 2003). The findings of this review were as follows:

- Hydrodynamic modeling has shown significant exchange of water and transport of sediment between Sinclair and Dyes Inlets; therefore, the two inlets should be treated as a single water body with respect to the TMDL study plan development. This is similar to the approach adopted for the fecal coliform TMDL study for the inlets.

- In the last decade, significant source control (pollution prevention, hazardous waste minimization, CSO reduction, remediation, etc), sediment cleanup, dredging, and capping activities have taken place in Sinclair Inlet, but much of the sediment chemistry data on which most of the 303(d) listings are based were collected prior to these activities.

- Two segments in Sinclair Inlet and one segment in Dyes Inlets are currently on the 1998 303(d) list because one or more metals (As, $\mathrm{Cd}, \mathrm{Cu}, \mathrm{Pb}, \mathrm{Ag}$, Zn, and/or $\mathrm{Hg}$ ) exceeded the Washington State SQS or MCUL (Washington State 1995)

The technical team concluded that the sediment data on which the 303(d) listings were based may not be representative of the present-day sediment conditions in Sinclair and Dyes Inlets, and recommended that representative data be collected before continuing work on the metals TMDL study plan. Furthermore, data that are spatially representative of the Sinclair and Dyes Inlet watershed are needed to support watershed-level contaminant modeling, which would also support TMDL development. The schematic diagram in Figure 6 shows how such data would be used to verify the need for metals TMDL planning. 


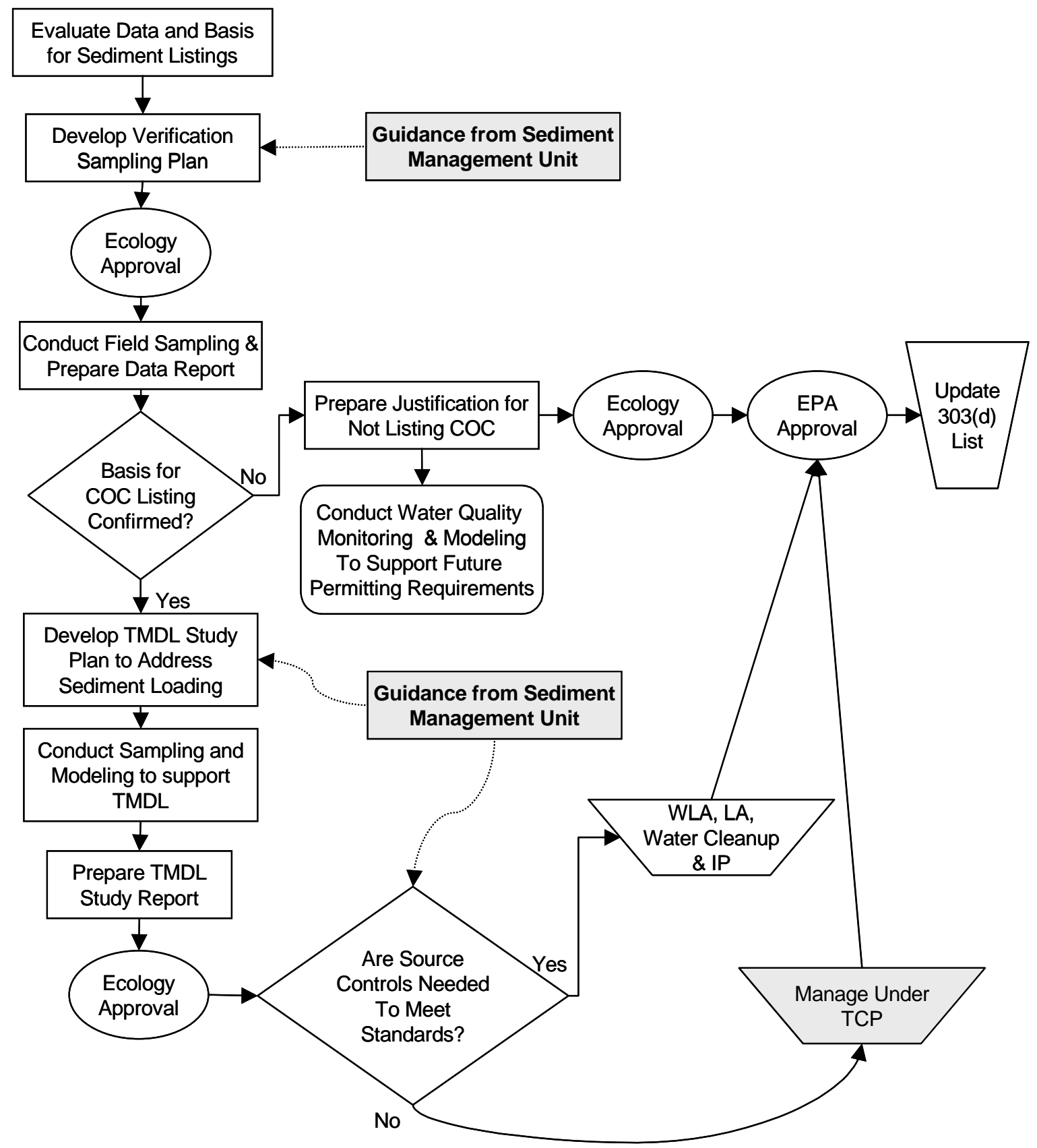

If a TMDL is needed for specific chemical of concern (COC), the total maximum daily loads (TMDL) study will result in the development of waste load allocations (WLA) for nonpoint sources, load allocations (LA) for point sources, and a water cleanup and implementation plan (IP) for the watershed (TCP = toxics cleanup program; $\mathrm{EPA}=$ U.S. Environmental Protection Agency; Ecology = Washington State Department of Ecology)

Figure 6. Schematic Drawing of the process to assess 303(d) metals listings in Sinclair and Dyes Inlets, Washington 
ENVVEST proceeded to develop and implement a sampling analysis plan for the Metals Verification Study (“develop verification sampling plan” step in Figure 6), the results of which are presented in this report. The Metals Verification Study was designed to answer the following questions:

- Have sediment metals concentrations in Sinclair and Dyes Inlets decreased since cleanup and source reduction actions?

- Do present-day concentrations of metals in Sinclair and Dyes Inlet sediment still exceed Washington State Sediment Quality Standards?

- If so, where? Should sediment remain on the 303(d) list because of metals?

Because the 303(d) listings for metals in Sinclair and Dyes Inlets are primarily due to sediment concentrations, the Metals Verification Study was guided by state sediment management sampling and analysis requirements to assure collection of appropriate samples and data with which to meet the state Water Quality Program Policy. In addition, hydrodynamic modeling has shown significant water exchange and sediment transport between Sinclair and Dyes Inlets. Another objective was to provide metals and particle size data to support contaminant loading, transport modeling, and sediment trends analysis throughout Sinclair and Dyes Inlets. The Metals Verification Study Sampling and Analysis Plan was presented to the Washington State Department of Ecology (Ecology), the U. S. Environmental Protection Agency (EPA), and other ENVVEST parties in July 2003; the plan was approved by Ecology in August 2003 (Kohn et al. 2003). 


\subsection{METHODS}

The Metals Verification Study approach was to collect and analyze surface sediment samples from throughout Sinclair and Dyes Inlets and the adjoining water bodies (Port Orchard Passage and Rich Passage). The study design and approaches for outside and inside Sinclair Inlet are described in Section 2.1. Sample collection and chemical analysis procedures are described in Sections 2.2 and 2.3.

\subsection{Metals Verification Study Design}

The overall study design for the Metals Verification Study considered a variety of research needs, primarily the needs of the state 303(d) and sediment management programs but also the existing monitoring program for OU B Marine and potential contaminant transport modeling. Specific design considerations for station placement were as follows:

- Higher sampling density in areas affected by dredging and cleanup activities (OU B Marine, Sinclair Inlet, Ostrich Bay)

- Proximity to known or potential sources

- Minimum of three samples in each 303(d) segment listed for metals in sediment

- Locations with recent existing data (i.e., from ENVVEST Mass Balance Study)

- Spatial coverage to support short- and long-term contaminant transport modeling

- Depositional vs. erosional areas.

Inside Sinclair Inlet, the Metals Verification Study obtained splits of over 100 surface sediment samples collected by the PSNS OU B Marine monitoring program, which allowed ENVVEST to avoid duplication of sampling effort and focus on optimizing the sampling design for Dyes Inlet and Port Orchard Passage outside of Sinclair Inlet. Sampling designs both inside and outside Sinclair Inlet are described below and summarized in Table 1.

The Metals Verification Study employed a tiered analytical approach to metals analysis in the large number of sediment samples generated for the study. The first step was to conduct rapid screening for a subset of target metals, followed by confirmatory analysis for all target metals in approximately 25\% of samples. Analytical methods and selection criteria for confirmatory samples are provided in Section 2.3.

\subsubsection{Sampling Design for Sinclair Inlet, OU B Marine}

In Sinclair Inlet, two segments are listed for metals in sediment on the 1998 303(d) list. A significant portion of the listed segments are within the boundary of PSNS OU B Marine, which is managed as a contaminated sediment site with oversight by Ecology’s Sediment Management Unit. A sediment 
Table 1. Metals Verification Study Design Summary for Sinclair and Dyes Inlets

\begin{tabular}{|c|c|c|c|}
\hline Location & Objective(s) & Approach $^{\mathrm{a}}$ & $\begin{array}{l}\text { Number of } \\
\text { Stations }^{b}\end{array}$ \\
\hline $\begin{array}{l}\text { Sinclair Inlet, Inside } \\
\text { PSNS OU-B }\end{array}$ & $\begin{array}{l}\text { 1. Present-day sediment quality in listed segments } \\
47122 \mathrm{~F} 6 \mathrm{~F} 3 \text { and } 47122 \mathrm{~F} 6 \mathrm{~F} 4 \text { that are within OU B } \\
\text { boundary } \\
\text { 2. Spatially representative data to support } \\
\text { contaminant transport modeling in Sinclair \& Dyes } \\
\text { Inlets }\end{array}$ & \multirow[t]{2}{*}{$\begin{array}{l}\text { Obtain and analyze an aliquot } \\
\text { of OU-B Marine monitoring } \\
\text { samples }^{\mathrm{a}, \mathrm{b}}\end{array}$} & 71 \\
\hline $\begin{array}{l}\text { Sinclair Inlet, } \\
\text { Outside PSNS OU-B }\end{array}$ & $\begin{array}{l}\text { 1. Present-day sediment quality throughout Sinclair } \\
\text { Inlet, outside OU-B boundary } \\
\text { 2. Spatially representative data to support } \\
\text { contaminant transport modeling in Sinclair \& Dyes } \\
\text { Inlets }\end{array}$ & & 32 \\
\hline & & Sinclair Inlet Subtotal & 103 \\
\hline Dyes Inlet & $\begin{array}{l}\text { 1. Present-day sediment quality in listed segment } \\
\text { 47122F6I8 (Ostrich Bay) } \\
\text { 2. Spatially representative data to support } \\
\text { contaminant transport modeling in Sinclair \& Dyes } \\
\text { Inlets }\end{array}$ & $\begin{array}{l}\text { Stratified design with stations } \\
\text { on triangular grid with random } \\
\text { start point. }\end{array}$ & 37 \\
\hline $\begin{array}{l}\text { Port Washington } \\
\text { Narrows }\end{array}$ & $\begin{array}{l}\text { 1. Data from potential source and depositional areas } \\
\text { to support contaminant transport modeling in Sinclair } \\
\text { \& Dyes Inlets }\end{array}$ & $\begin{array}{l}\text { Use existing data from outfalls } \\
\text { sampled during Mass Balance } \\
\text { Study }\end{array}$ & $\begin{array}{c}1 \\
\text { (Phinney Bay) }\end{array}$ \\
\hline $\begin{array}{l}\text { Port Orchard Passage } \\
\text { at confluence of Port } \\
\text { Washington Narrows } \\
\text { and Sinclair Inlet }\end{array}$ & $\begin{array}{l}\text { 1. Spatially representative data to support } \\
\text { contaminant transport modeling in Sinclair \& Dyes } \\
\text { Inlets }\end{array}$ & $\begin{array}{l}\text { Stations on triangular grid with } \\
\text { random start point within } \\
\text { defined area of interest }\end{array}$ & 17 \\
\hline $\begin{array}{l}\text { North Port Orchard } \\
\text { Passage and Rich } \\
\text { Passage }\end{array}$ & $\begin{array}{l}\text { 1. Sediment quality of locations not impacted by } \\
\text { contaminants from Sinclair and Dyes Inlets, to } \\
\text { initialize model parameters }\end{array}$ & $\begin{array}{l}\text { Existing ENVVEST stations or } \\
\text { new judgment stations }\end{array}$ & 4 \\
\hline
\end{tabular}

a Analytical approach for all samples is to analyze all samples for metals by XRF rapid screening technique, then use ICP-MS to confirm metals concentrations in $\sim 25 \%$ of samples.

b 103 Sinclair Inlet samples to be collected by EFA NW under OUB Marine Monitoring; samples outside of Sinclair Inlet to be collected by Battelle/ENVVEST team.

management plan (OU B Marine Monitoring Program) is in place for OU B; the proposed monitoring area extent covers all of Sinclair Inlet. Post-remediation monitoring for OU B Marine focused on the goals of the Record of Decision (ROD) (U.S. Navy 2000a). The goals of the ROD were to (1) reduce the area-weighted concentration of PCBs to the minimum cleanup goal of $3 \mathrm{mg}$ PCB per $1 \mathrm{~kg}$ of organic carbon (3 mg PCB/kg OC) within 10 years; (2) selectively remove high concentrations of Hg collocated with PCBs; and (3) control shoreline erosion of contaminated fill (U.S. Navy 2000a).

The ENVVEST Metals Verification Study coordinated with the OU B Marine Monitoring Program to maximize sample distribution (high density) and data utility, and to take advantage of the monitoring 
program's sample collection effort. The OU B Marine monitoring design consisted of intensive grab sampling at three stations in each of 71500 -ft grids within the OU B Marine boundary (OUBM), and at three stations in each of 32 1500-ft grids throughout Sinclair Inlet outside the OU B Marine boundary (OOUB) (Figures 7 and 8). The 0 - to 10 -cm surface sediment interval from each of the three stations was combined to form a composite sample representing the grid in which the grabs were collected. Although the OU B Marine Monitoring Program spatially covered all of Sinclair Inlet, its analyte list did not include any of the target metals (As, $\mathrm{Cd}, \mathrm{Cu}, \mathrm{Pb}, \mathrm{Ag}$, or $\mathrm{Zn}$ ). The Metals Verification Study obtained an aliquot of each OUBM and OOUB composite sample for target metal analysis.

\subsubsection{Dyes Inlet, Ostrich Bay, Port Orchard Passage, and Rich Passage}

A different sampling strategy was required in the portion of the Sinclair-Dyes Inlet study area that is not included in the OU B Marine Monitoring Program. In this area, only two segments off Jackson Park Housing Complex are presently listed. Therefore, sample collection in Dyes Inlet, Port Washington Narrows, and Port Orchard Passage outside of OU B Marine is primarily to support contaminant transport modeling efforts as well as verify ambient sediment quality in the study area. To adequately describe the sediment ecosystem in the model, more sampling data points are needed where sediment is expected to accumulate, because that is where metals concentrations are expected to be most variable. Sediment depositional areas may not be near known sources, but sediment transport into Dyes Inlet could result in higher concentrations in sediment areas removed from direct sources. Fewer sampling data points are needed in erosional areas and areas for which recent sediment data are available. Data from Port Orchard and Rich Passages are needed to initialize model parameters for the entire model domain.

A stratified random sampling design was developed with three strata defined as follows:

- $\quad 303(d)-l i s t e d ~ s e g m e n t(s) ~ \Rightarrow$ highest sampling density

- Depositional areas or close to PSNS $\Rightarrow$ moderate sampling density

- $\quad$ Non-depositional areas, passages, sample for spatial extent $\Rightarrow$ lower sampling density

Within each stratum, sampling locations on a triangular grid from a random starting point were selected using Visual Sampling Plan software (Ver. 2) developed by Pacific Northwest National Laboratory

(Figure 9). Ostrich Bay in the vicinity of Jackson Park was the only area outside of Sinclair Inlet in the highest sampling density stratum. The depositional west side of Dyes Inlet was considered the moderatedensity sampling area; the remaining areas of Dyes Inlet, Port Orchard Passage, and Rich Passage all fell into the lower sampling density stratum. Figure 9 also shows the locations of "core and grab" stations for which recent metals data are available from the ENVVEST Mass Balance Study. 


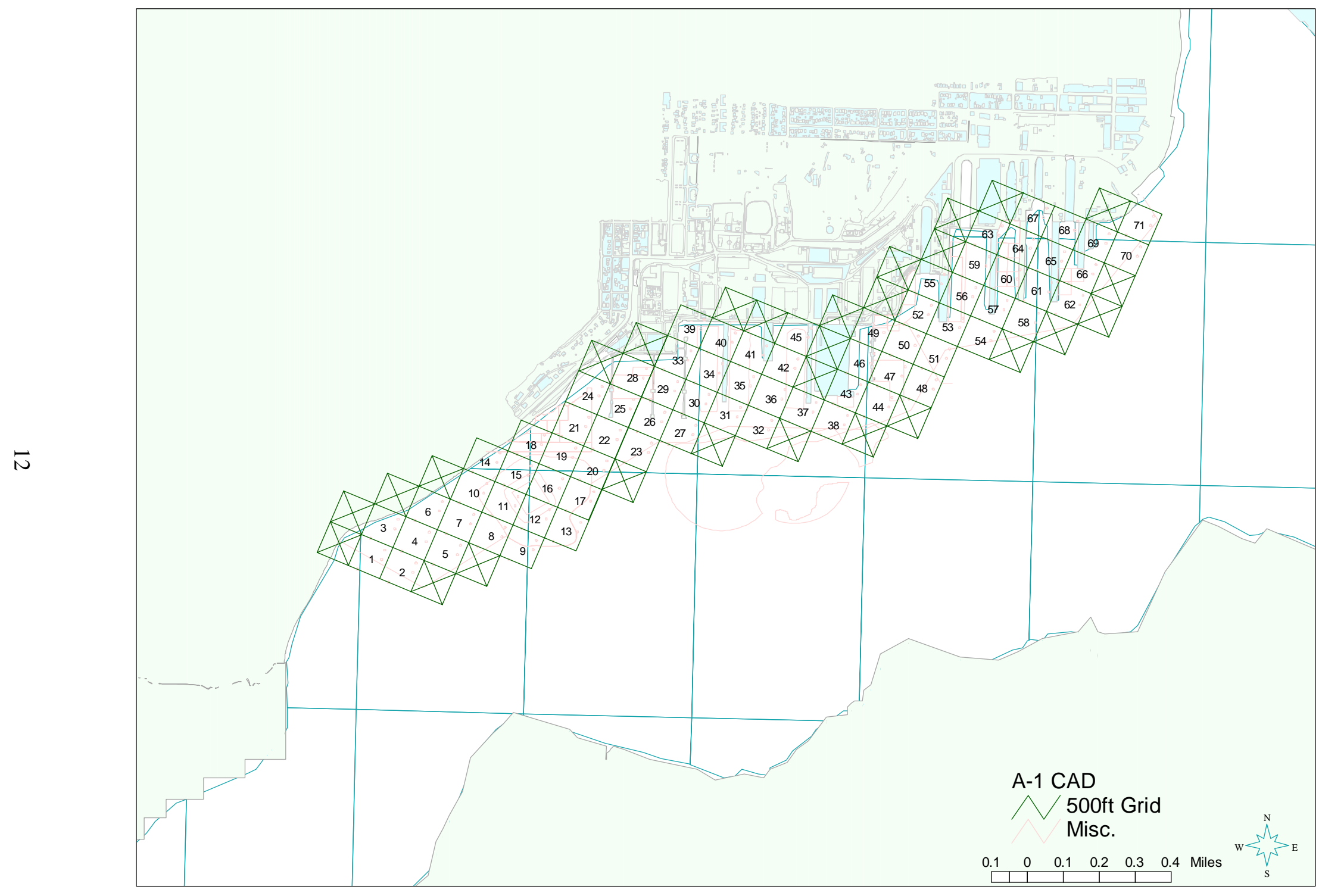

Figure 7. 500-ft monitoring grids within the OU B Marine boundary (OUBM), with 303(d) segment overlay (blue) 


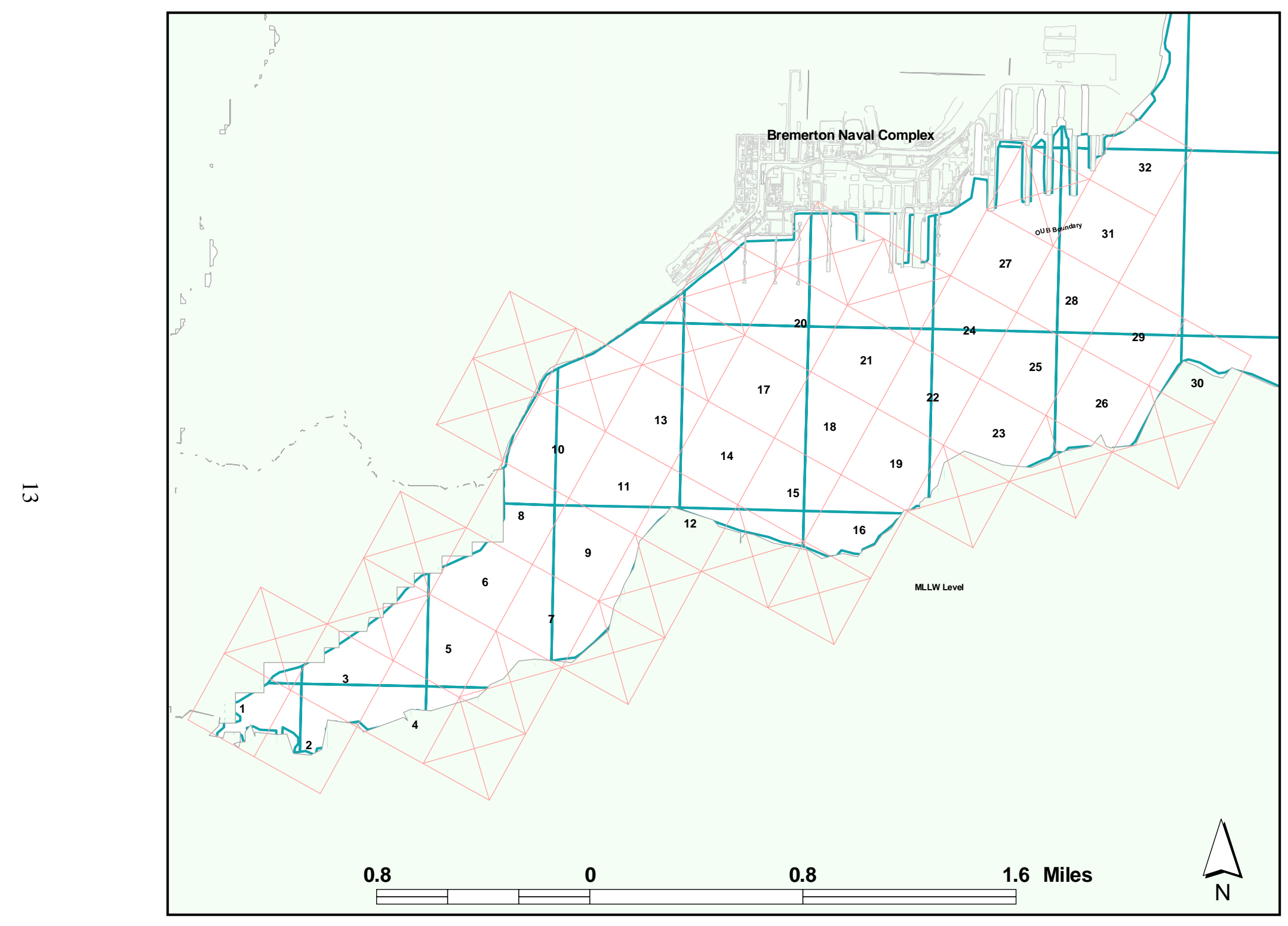

Figure 8. 1500-ft monitoring grids outside of the OU B Marine boundary (OOUB), with 303(d) segment overlay (blue) 


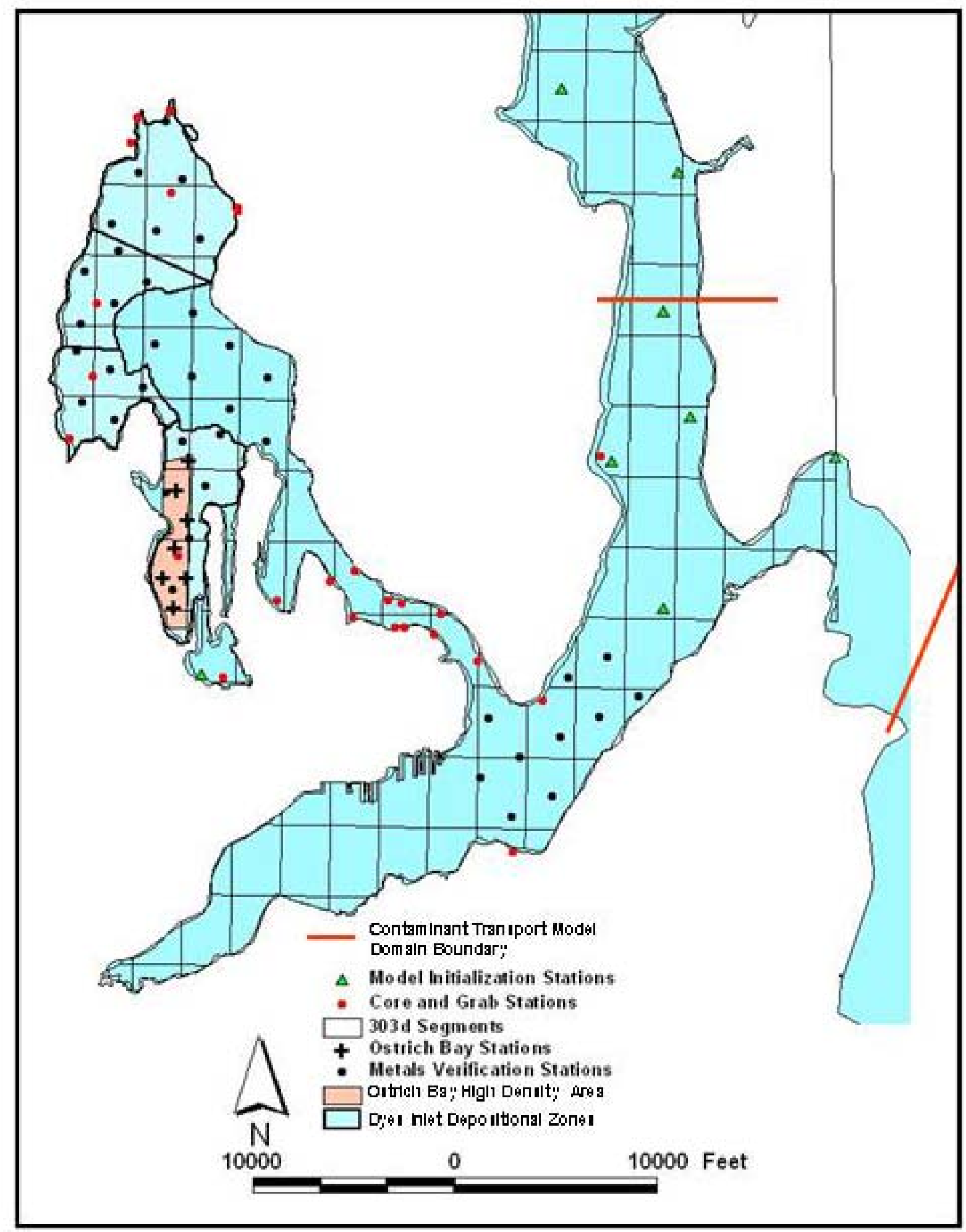

Figure 9. Planned Metals Verification Study (black dots and crosses) and model initialization (green triangles) stations in Dyes Inlet, Port Washington Narrows, and Port Orchard Passage 


\subsection{Sediment Sample Collection}

Surface sediment samples outside of Sinclair Inlet were collected by Project ENVVEST, August 28-31, 2003. Samples were collected using a van Veen grab sampler deployed from the Battelle Marine Sciences Laboratory (MSL) research vessel Strait Science. Navigation to each planned location was by a differentially-corrected global positioning system (dGPS) aboard the sampling vessel. The van Veen grab sampler allows collection of undisturbed surficial sediment to a depth of up to $15 \mathrm{~cm}$. The 0 - to $10-\mathrm{cm}$ interval was removed from the sampler using a clean stainless steel spoon and placed in a certified precleaned, labeled 1-gal wide-mouth high-density polyethylene (HDPE) container (Figure 10). HDPE sample containers were placed in a cooler with ice until transfer to the MSL's walk-in cold room.

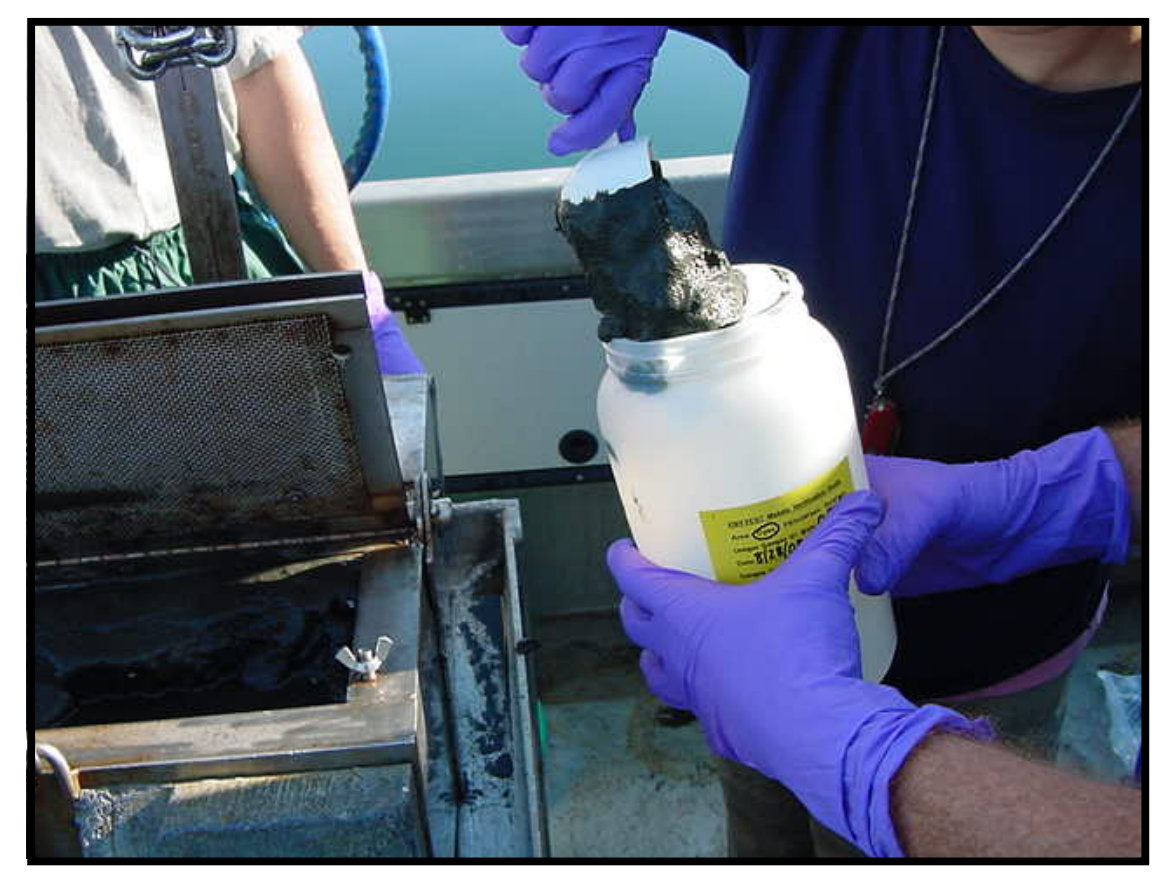

Figure 10. Metals Verification Study grab sample collection

Generally one grab per station yielded a sufficient quantity of sediment for the proposed analyses and archive samples. Surface sediment sample cross-contamination was avoided by cleaning equipment thoroughly between sampling stations, and collecting only sample material that was not in direct contact with sample collection equipment. The grab sampler was scrubbed with a stiff brush and thoroughly rinsed with site water at the beginning of each day and between each sampling station. Sampling methods were consistent with the Puget Sound Estuary Program (PSEP) Recommended Guidelines for Sampling Marine Sediment, Water Column, and Tissue in Puget Sound (PSEP1997), and the more recent EPA document, Methods for Collection, Storage and Manipulation of Sediments for Chemical and Toxicological Analyses: Technical Manual (EPA 2001). 
Sediment samples from Sinclair Inlet were collected by a Navy contractor, URS Corporation, in October 2003. Field sampling methods for Sinclair Inlet are detailed in the Draft Long Term Monitoring Plan, OU B Marine Bremerton Naval Complex (URS 2002b). URS also collected samples using a van Veen grab sampler and removing the 0 - to 10 -cm surface interval. EFANW/URS provided an aliquot (approximately $400 \mathrm{~mL}$ ) of homogenized composite sample for each OUBM and OOUB monitoring grid to Project ENVVEST.

\subsection{Chemical Analysis}

The target analytes for the Metals Verification Study, identified in Diefenderfer et al. (2003), are As, Cd, $\mathrm{Cu}, \mathrm{Pb}, \mathrm{Ag}$ and $\mathrm{Zn}$. Listings for As are primarily based on observed tissue levels in marine biota, rather than in sediment. However, As was measured in all confirmatory Metals Verification Study sediment samples. Hg was not addressed in the Metals Verification Study for several reasons. In general, sediment $\mathrm{Hg}$ in Sinclair and Dyes Inlets is due to historical releases, rather than to ongoing or active discharges, and would likely be managed under a different program. Hg chemistry is more complex, and Hg risk is mainly associated with biota that uptake and accumulate methylmercury; Hg risk management would likely include sampling and analysis of biota as well as sediment. In Sinclair Inlet, $\mathrm{Hg}$ is being addressed by the OU B Marine Monitoring Program, and it is well documented that ambient Hg concentrations exceed sediment quality standards (Miller et al. 2003); therefore, verification sampling for Hg is not needed at this time.

The Metals Verification Study employed the following tiered analytical approach:

- Tier 1 - Rapid screening analysis of all samples by X-ray fluorescence (XRF), conducted by the Space and Naval Warfare Systems Center (SSC), San Diego, California

- Tier 2 - Confirmatory analysis of at least $25 \%$ of samples by inductively coupled plasma-mass spectrometer (ICP-MS), conducted by the MSL, Sequim, Washington

- Methods for each are described below.

Homogenized sediment from each Metals Verification Study sample was split into two labeled precleaned sample jars: a 4-oz glass jar with a Teflon-lined cap for metals screening analysis and a 16-oz glass jar with a Teflon-lined cap for archiving and other laboratory analyses. The 4-oz jar was shipped immediately to SSC; the 16-oz jar was held frozen at the MSL pending selection for ICP-MS or other ancillary analyses. 


\subsection{1 $\quad \underline{\text { X-Ray Fluorescence Screening for Metals }}$}

All sediment samples collected in the Metals Verification Study were screened by XRF (modified EPA Method 6200 [EPA 1998]) at the SSC laboratory to determine the range of levels of the metals Ag, Cd, $\mathrm{Cu}, \mathrm{Pb}$ and $\mathrm{Zn}$. Samples are exposed to X-ray energy, which liberates electrons in the inner shell of metal atoms. As the outer electrons cascade toward the inner shells to fill the vacancies, energy is released (fluorescence). The fluorescing energy spectrum identifies the metals and the intensity is proportional to concentration. Inorganic analytes of interest are identified and quantitated using a QuanX EDXRF Spectrometer (Spectrace Instruments, Sunnyvale, California). This instrument contains an Rh-anode Xray tube for primary generation of X-rays $(4-50 \mathrm{kV})$ and a thermoelectrically cooled, solid-state Silicon (Si) (lithium [Li]) detector. The $\mathrm{Si}(\mathrm{Li})$ detector provides spectral resolution that exceeds that of other solid-state detectors or gas-filled proportional detectors. Quality control (QC) analyses to address accuracy and precision of XRF measurements included standard reference material samples and laboratory triplicate samples.

Historically, $\mathrm{Pb}, \mathrm{Cu}$, and Zn, concentrations measured by XRF show very good correlation with concentrations measured by ICP-MS (Figure 11, Miller et al. 2003), and reliable XRF detection limits for those metals are well below the state management standard concentrations (Table 2). However, XRF detection limits for Cd are close to the SQS concentration, and the XRF detection limit for Ag is greater than the SQS, to allow comparison (Table 2). For this reason, confirmatory ICP-MS analysis was done on at least three samples from segments listed for Cd or Ag.
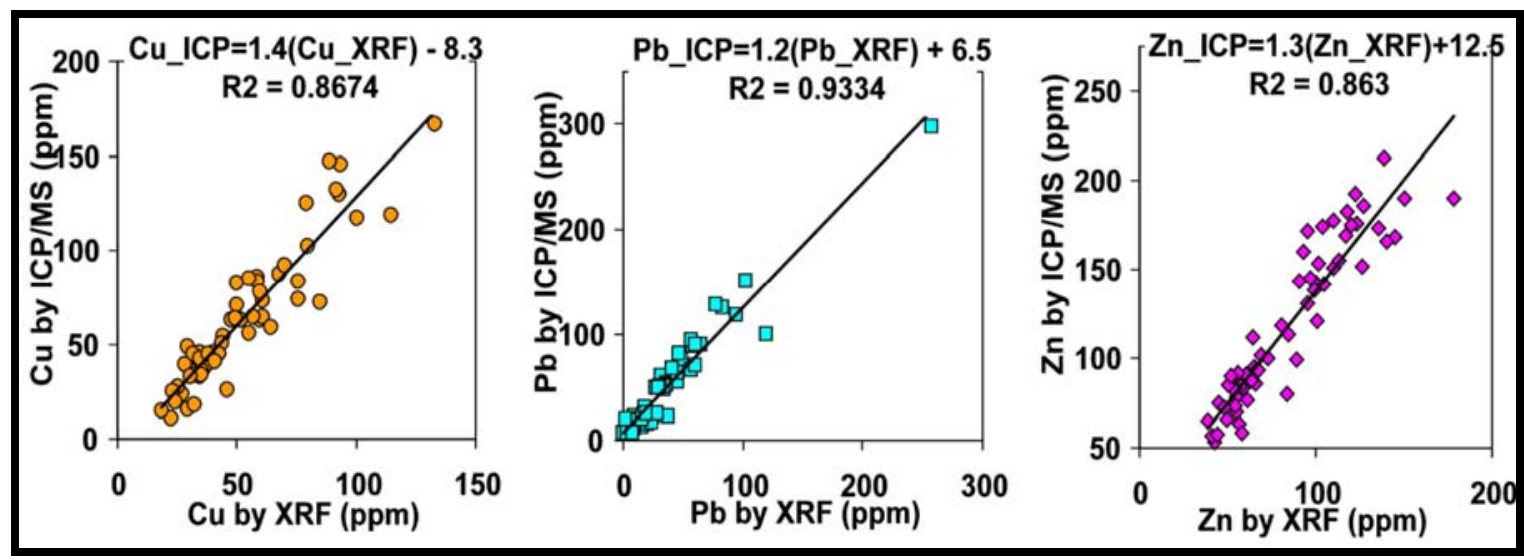

Figure 11. Linear correlation of XRF and ICP-MS Cu, Pb, and Zn measurements 
Table 2. XRF Screening and ICP-MS Detection Limits Compared with State Sediment Management Standards for ENVVEST Metals of Concern

\begin{tabular}{|c|c|c|c|c|c|}
\hline \multirow[b]{2}{*}{ Analyte } & \multirow[b]{2}{*}{ Units } & \multirow{2}{*}{\begin{tabular}{|c|} 
Reliable Detection \\
Limit for XRF
\end{tabular}} & \multirow{2}{*}{$\begin{array}{l}\text { Method Detection } \\
\text { Limit for ICP/MS }^{\text {a }}\end{array}$} & \multicolumn{2}{|c|}{$\begin{array}{l}\text { Washington State Sediment } \\
\text { Management Standards }\end{array}$} \\
\hline & & & & SQS & MCUL \\
\hline $\mathrm{Ag}$ & $\mathrm{mg} / \mathrm{kg}$ dry wt & 10.0 & 0.013 & 6.1 & 6.1 \\
\hline As & $\mathrm{mg} / \mathrm{kg}$ dry wt & 20.0 & 0.7 & 57 & 93 \\
\hline $\mathrm{Cd}$ & mg/kg dry wt & 5.0 & 0.01 & 5.1 & 6.7 \\
\hline $\mathrm{Cr}$ & mg/kg dry wt & 100 & 0.2 & 260 & 270 \\
\hline $\mathrm{Cu}$ & mg/kg dry wt & 18.0 & 0.09 & 390 & 390 \\
\hline $\mathrm{Ni}$ & mg/kg dry wt & 50.0 & 0.02 & None & none \\
\hline $\mathrm{Pb}$ & mg/kg dry wt & 8.0 & 0.03 & 450 & 530 \\
\hline $\mathrm{Zn}$ & $\mathrm{mg} / \mathrm{kg}$ dry wt & 16.0 & 0.2 & 410 & 960 \\
\hline
\end{tabular}

a. Updated according to SOP MSL-I-022, Determination of Elements in Aqueous and Digestate Samples by ICP/MS, and MSL-Q-007, Procedure for Determining Method Detection Limits.

\subsubsection{Confirmatory Analysis of Metals}

Following XRF screening of all Metals Verification Study samples, 25\% of the samples were selected for confirmatory ICP-MS analysis using the following criteria:

- samples in which XRF result exceeds $90 \%$ of the SQS for one or more target metals $(\mathrm{Cu}, \mathrm{Pb}$, and $\mathrm{Zn})$

- $\quad$ at least 3 samples in 303(d) segments listed for Cd or Ag (because XRF screening does not detect these metals at levels comparable to state SQS)

- samples in which XRF result was different (much higher or lower) than expected

- additional samples to represent range of concentrations observed for each target metal, particularly at high end of range where little XRF:ICP-MS correlation data exist.

ICP-MS is a high-resolution multi-element determination method with detection levels well below Washington State SQS (Table 2). Freeze-dried sediment samples are acid-digested; material in solution (digestate) is introduced by pneumatic nebulization into a radio-frequency plasma where energy transfer processes cause desolvation, atomization, and ionization. The ions are extracted from the plasma through a differentially pumped vacuum interface and separated on the basis of their mass-to-charge ratio by a quadrupole mass spectrometer having a minimum resolution capability of 1 amu peak width at $5 \%$ peak height. The ions transmitted through the quadrupole are detected by a continuous dynode electron multiplier assembly and the ion information processed by a data handling system. Interferences, instrumental drift, and suppressions or enhancements of instrument response caused by the sample matrix are corrected for by using internal standardization. 
All target metals were analyzed by ICP-MS. Ag was also analyzed by graphite furnace atomic absorption (GFAA). GFAA results for Ag are reported here for comparability with data generated for other Project ENVVEST studies and stored in the ENVVEST database (i.e., mass balance, stormwater loading). QC samples to assess accuracy and precision of quantitative measurements included method blanks, laboratory duplicates, matrix spikes, standard reference materials, and laboratory control samples (blank spikes).

The Metals Verification Study XRF screening and quantitative metals concentrations were plotted to develop Sinclair-Dyes Inlet site-specific linear correlations. As in Figure 11, good linear relationships are expected between screening and quantitative measurements of at least three of the target metals $(\mathrm{Cu}, \mathrm{Pb}$, and $\mathrm{Zn}$ ). The linear correlation equations were used to calculate definitive metal concentrations from screening concentrations in all Metals Verification Study samples.

\subsubsection{Ancillary Sediment Analyses Outside of Metals Verification Study}

Total Hg was not measured as part of the Metals Verification Study. Total Hg concentrations, grain size, and total organic carbon (TOC) in Sinclair Inlet (OUBM and OOUB) samples will be provided by the OU B Marine Monitoring Program. However, Project ENVVEST provided an aliquot of each Metals Verification Study sample from outside Sinclair Inlet for Hg analysis by Ecology’s Manchester Environmental Laboratory. The Metals Verification Study also provided aliquots of sediment to GeoSea, Inc., for particle size analysis by laser diffraction; the purpose of these data was to support sediment trends analysis outside of the Metals Verification Study. Remaining Metals Verification Study samples continue to be archived frozen for possible later analysis of organics (PCB, PAH). 


\subsection{RESULTS AND DISCUSSION}

\subsection{Sample Collection}

\subsubsection{Dyes Inlet, Ostrich Bay, Port Orchard Passage, and Rich Passage}

Fifty-seven sediment samples were collected from Dyes Inlet, Ostrich Bay, Port Orchard Passage, and Rich Passage by the Project ENVVEST technical team August 28-31, 2003. Sampling locations and sediment information are provided in Figures 12 and 13 and in Table 3. Most samples were successfully collected at or very close to the target sampling location. Several stations were moved slightly to reach navigable water or penetrable sediment. Two target stations at the confluence of Port Orchard and Rich Passages were attempted numerous times but were abandoned because the substrate of large rocks or shells could not be successfully sampled; such large-particle substrate indicates a high-energy environment where contaminants are unlikely to accumulate.

\subsubsection{Sinclair Inlet}

A total of 103 aliquots of Sinclair Inlet sediment composites was provided by the OU B Marine Monitoring team. OUBM 500-ft grid sampling locations are shown in Figure 14; OOUB 1500-ft grid sampling locations are shown in Figure 15. In these figures, the three light pink dots indicate the actual grab locations, and the single magenta dot is assigned to the geographical average location used to represent the composite sample received by the Metals Verification Study. Composite samples from grids marked with gold circles received confirmatory metals analysis by ICP-MS. A list of OU B Marine monitoring samples provided to Metals Verification Study and cross-referenced to 303(d) grids is provided in Table 4.

\subsection{Sediment Metals Chemistry}

All Metals Verification Study samples from outside Sinclair Inlet and all samples from Sinclair Inlet provided by the OU B Marine monitoring program were screened for $\mathrm{Cu}, \mathrm{Pb}, \mathrm{Zn}, \mathrm{Cd}$, and $\mathrm{Ag}$ by XRF. XRF screening results for all 160 samples are provided in Table 5. The laboratory data report for XRF analysis, including narrative summary and QC data, is provided in Appendix A. XRF results for standard reference materials were $1 \%$ to $25 \%$ different from the certified value for all metals except Cd. Two of four Cd percent differences were higher (33\%, 49\%) because the XRF quantitation limit is not as low as the certified value for $\mathrm{Cd}$ in the standard reference material. For all metals detected above the quantitation limit, relative standard deviations between triplicate samples were $<20 \%$, indicating acceptable laboratory precision. 


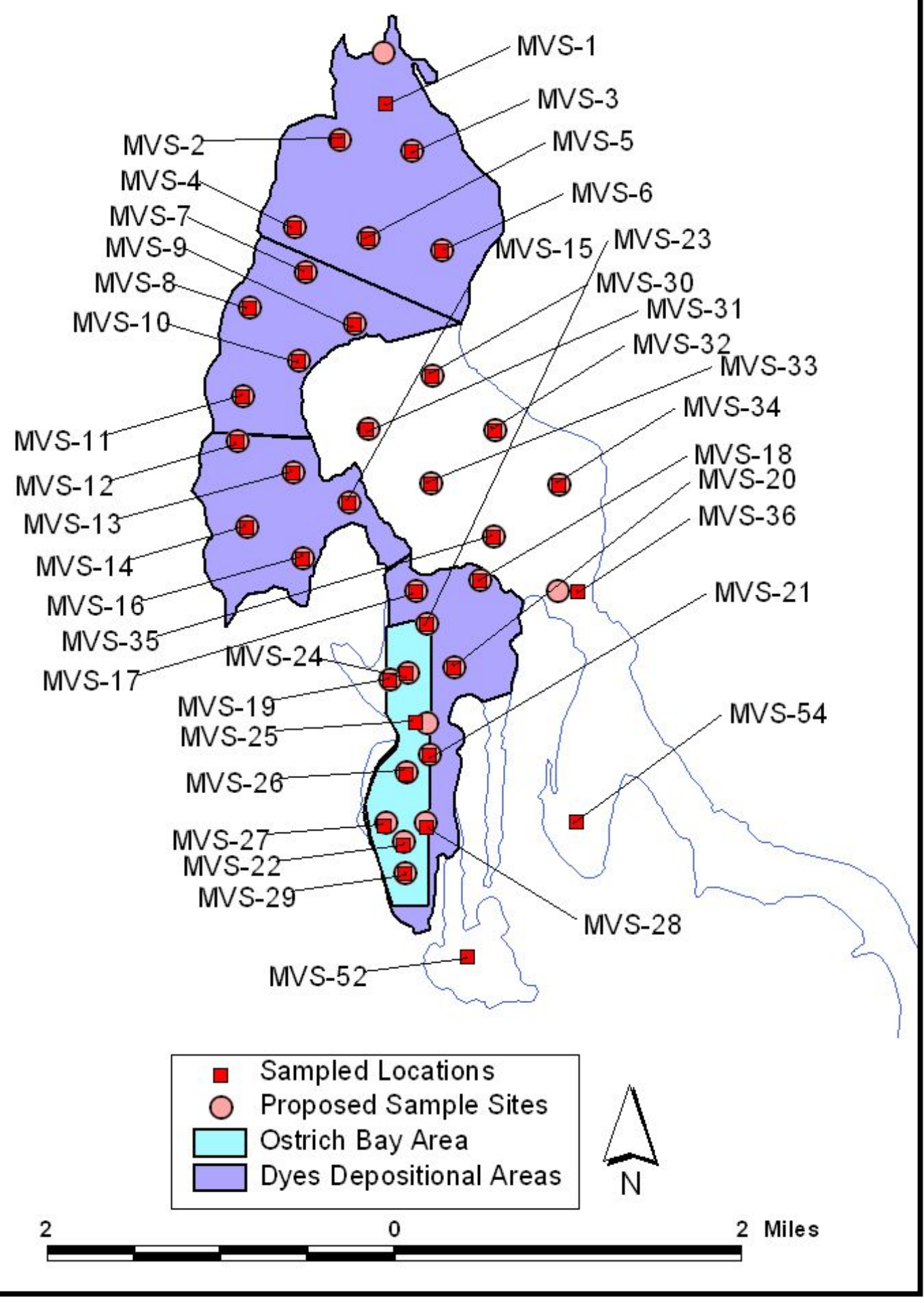

Figure 12. Metals Verification Study stations sampled in Dyes Inlet, including Ostrich Bay 


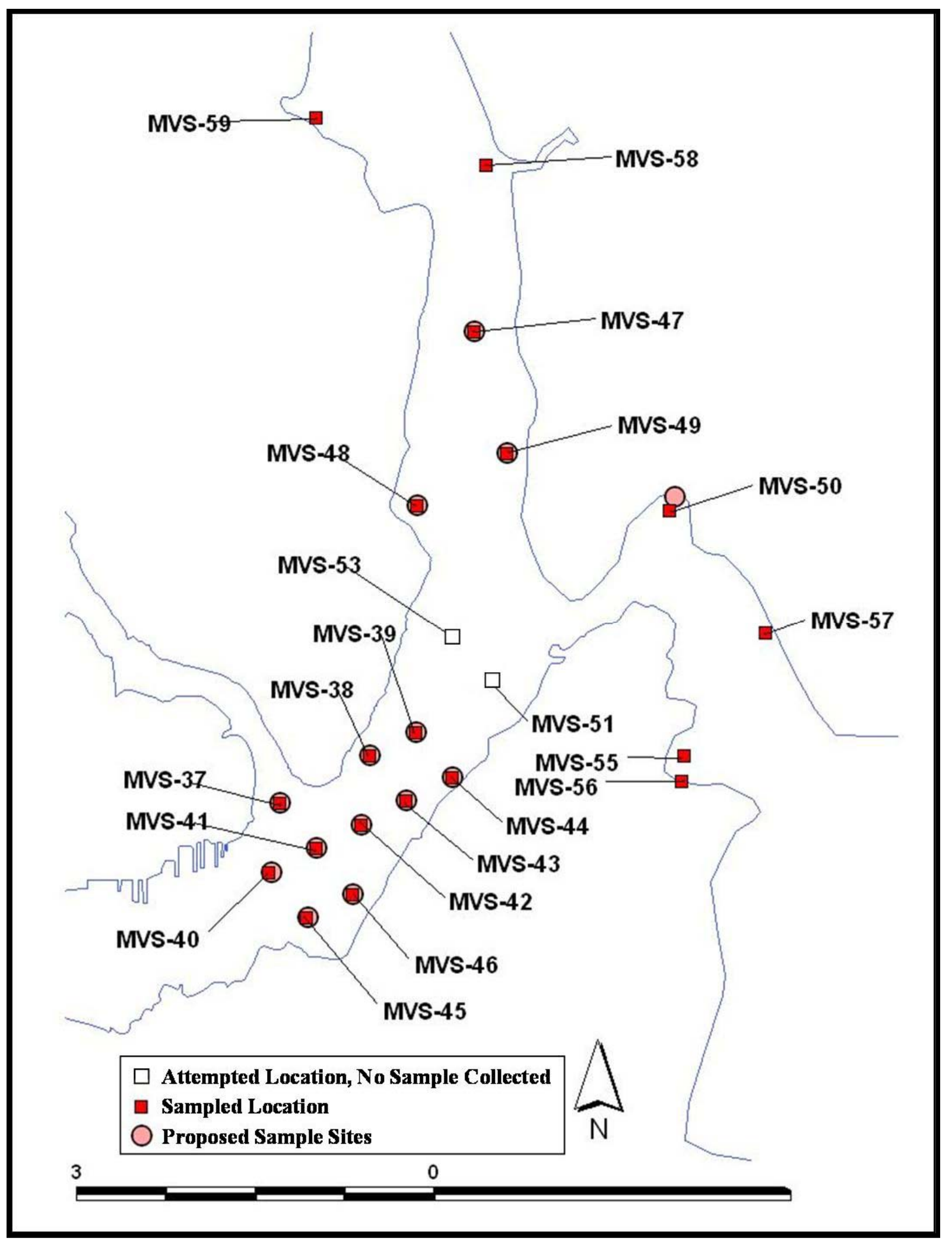

Figure 13. Metals Verification Study stations sampled in Port Orchard Passage and Rich Passage 
Table 3. Metals Verification Study Sample Collection Information for Dyes Inlet, Port Orchard Passage, and Rich Passage

\begin{tabular}{|c|c|c|c|c|c|c|c|c|c|}
\hline \multirow[b]{3}{*}{ Area } & \multirow{3}{*}{$\begin{array}{c}\text { MVS } \\
\text { Sample ID }\end{array}$} & \multirow{2}{*}{\multicolumn{2}{|c|}{ Collection }} & \multirow{3}{*}{$\begin{array}{c}\text { Water } \\
\text { Depth } \\
\text { (ft, Un- } \\
\text { corrected) }\end{array}$} & \multirow{2}{*}{\multicolumn{2}{|c|}{$\begin{array}{c}\text { Coordinates } \\
\text { (dd mm.mmm, NAD 83) }\end{array}$}} & \multirow{3}{*}{$\begin{array}{l}\text { Maximum } \\
\text { Penetration } \\
\text { Depth }(\mathbf{c m}) \\
\end{array}$} & \multirow{3}{*}{$\begin{array}{c}\text { Sampled } \\
\text { Interval } \\
\text { (cm) }\end{array}$} & \multirow[b]{3}{*}{ Comments } \\
\hline & & & & & & & & & \\
\hline & & Date & Time & & Latitude & Longitude & & & \\
\hline Dyes Depositional Zone 1 (North) & MVS- 001 & 08/29/03 & 1518 & 7 & $47^{\circ} 38.696$ & $122^{\circ} 41.186$ & 15 & $0-10$ & $\begin{array}{l}\text { Station located in as shallow water possible, between } \\
\text { and north of MVS- } 002 \text { and MVS-003. Dark gray } \\
\text { medium-fine sand with wood chips, slight H2S odor. }\end{array}$ \\
\hline Dyes Depositional Zone 1 (North) & MVS- 002 & $08 / 29 / 03$ & 1358 & 13 & $47^{\circ} 38.513$ & $122^{\circ} 41.525$ & 12 & $0-10$ & $\begin{array}{l}\text { Gray-brown medium sand with } 0.5 \mathrm{~cm} \text { fines on } \\
\text { surface, worm tubes, worms, snails. }\end{array}$ \\
\hline Dyes Depositional Zone 1 (North) & MVS- 003 & 08/29/03 & 1442 & 27 & $47^{\circ} 38.457$ & $122^{\circ} 40.986$ & 15 & $0-10$ & $\begin{array}{l}\text { Dark gray-black clay, unconsolidated, few worms on } \\
\text { top. Strong H2S odor. }\end{array}$ \\
\hline Dyes Depositional Zone 1 (North) & MVS- 004 & $08 / 29 / 03$ & 1339 & 29 & $47^{\circ} 38.074$ & $122^{\circ} 41.842$ & 12 & $0-10$ & Dark gray-brown silt clay, slight H2S odor. \\
\hline Dyes Depositional Zone 1 (North) & MVS- 005 & $08 / 28 / 03$ & 1305 & 32.6 & $47^{\circ} 38.018$ & $122^{\circ} 41.304$ & 15 & $0-10$ & Dark gray clay, moderate H2S odor. \\
\hline Dyes Depositional Zone 1 (North) & MVS- 006 & 08/29/03 & 1502 & 28 & $47^{\circ} 37.962$ & $122^{\circ} 40.766$ & 13 & $0-10$ & $\begin{array}{l}\text { Gray medium stiff clayey sand, few worm tubes, no } \\
\text { odor. }\end{array}$ \\
\hline Dyes Depositional Zone 2 (West) & MVS- 007 & $08 / 29 / 03$ & 1327 & 32 & $47^{\circ} 37.848$ & $122^{\circ} 41.760$ & 15 & $0-10$ & $\begin{array}{l}\text { Brown silt-clay, very soft and unconsolidated, slight } \\
\text { H2S odor. }\end{array}$ \\
\hline Dyes Depositional Zone 2 (West) & MVS- 008 & 08/29/03 & 1312 & 30 & $47^{\circ} 37.664$ & $122^{\circ} 42.170$ & 12 & $0-10$ & Dark gray slit clay, slight H2S odor. \\
\hline Dyes Depositional Zone 2 (West) & MVS- 009 & $08 / 28 / 03$ & 1322 & 45 & $47^{\circ} 37.587$ & $122^{\circ} 41.400$ & 15 & $0-10$ & Gray-brown silty sand on dark gray-brown mud. \\
\hline Dyes Depositional Zone 2 (West) & MVS- 010 & 08/29/03 & 1255 & 52 & $47^{\circ} 37.403$ & $122^{\circ} 41.808$ & 9 & $0-8$ & $\begin{array}{l}\text { Black fine sand, silt, clay, slight H2S odor, lots of } \\
\text { biota, worm tubes. }\end{array}$ \\
\hline Dyes Depositional Zone 2 (West) & MVS- 011 & 08/29/03 & 1236 & 38 & $47^{\circ} 37.220$ & $122^{\circ} 42.212$ & 13 & $0-10$ & Dark gray-brown fine sand and silt, slight H2S odor. \\
\hline Dyes Depositional Zone 3 (SW) & MVS- 012 & $08 / 29 / 03$ & 1224 & 35 & $47^{\circ} 36.991$ & $122^{\circ} 42.256$ & 15 & $0-10$ & Dark gray-brown silt clay, slight H2S odor. \\
\hline Dyes Depositional Zone 3 (SW) & MVS- 013 & 08/29/03 & 1206 & 43 & $47^{\circ} 36.842$ & $122^{\circ} 41.841$ & 15 & $0-10$ & $\begin{array}{l}\text { Dark gray-brown silt clay with some fine sand, slight } \\
\text { H2S odor. Tunicate on surface. } \\
\end{array}$ \\
\hline Dyes Depositional Zone 3 (SW) & MVS- 014 & $08 / 29 / 03$ & 1154 & 16 & $47^{\circ} 36.560$ & $122^{\circ} 42.176$ & 14.5 & $0-10$ & $\begin{array}{l}\text { Dark gray fine clayey sand, shells, worm tubes, live } \\
\text { snails, moderate H2S odor. }\end{array}$ \\
\hline Dyes Depositional Zone 3 (SW) & MVS- 015 & 08/29/03 & 1122 & 16 & $47^{\circ} 36.693$ & $122^{\circ} 41.429$ & 6 & $0-5$ & Gray medium-fine sand with shells, no odor. \\
\hline Dyes Depositional Zone 3 (SW) & MVS- 016 & 08/29/03 & 1138 & 14 & $47^{\circ} 36.411$ & $122^{\circ} 41.767$ & 15 & $0-10$ & $\begin{array}{l}\text { Dark gray clay, very cohesive, slight H2S odor, } \\
\text { worm tubes. }\end{array}$ \\
\hline Dyes Depositional Zone 4 (South) & MVS- 017 & $08 / 28 / 03$ & 1525 & 42 & $47^{\circ} 36.244$ & $122^{\circ} 40.938$ & 14 & $0-10$ & Dark gray-brown silt and clay. \\
\hline Dyes Depositional Zone 4 (South) & MVS- 018 & 08/29/03 & 1100 & 47 & $47^{\circ} 36.304$ & $122^{\circ} 40.464$ & 10 & $0-9$ & $\begin{array}{l}\text { Dark gray-brown silt and clay, moderate H2S odor, } \\
\text { no biota. }\end{array}$ \\
\hline Dyes Depositional Zone 4 (South) & MVS- 019 & 08/28/03 & 1601 & 8.4 & $47^{\circ} 35.804$ & $122^{\circ} 41.122$ & 12 & $0-10$ & Dark gray silt clay. \\
\hline Dyes Depositional Zone 4 (South) & MVS- 020 & 08/29/03 & 1045 & 25 & $47^{\circ} 35.864$ & $122^{\circ} 40.648$ & 12 & $0-10$ & $\begin{array}{l}\text { Dark gray-brown silt clay, no odor, } 2 \text { crabs, hydroid } \\
\text { mats. }\end{array}$ \\
\hline Dyes Depositional Zone 4 (South) & MVS- 021 & 08/28/03 & 1640 & 24.8 & $47^{\circ} 35.424$ & $122^{\circ} 40.827$ & 15 & $0-10$ & $\begin{array}{l}\text { Dark gray silt clay, cohesive, no odor. Small } \\
\text { jellyfish on sediment surface. }\end{array}$ \\
\hline Dyes Depositional Zone 4 (South) & MVS- 022 & 08/29/03 & 0946 & 23.0 & $47^{\circ} 34.976$ & $122^{\circ} 41.012$ & 9 & $0-8$ & $\begin{array}{l}\text { Moved position slightly for third attempt which was } \\
\text { successful; dark gray-brown fine sand, silt, clay, } \\
\text { slight H2S odor. }\end{array}$ \\
\hline
\end{tabular}


Table 3. (continued)

\begin{tabular}{|c|c|c|c|c|c|c|c|c|c|}
\hline \multirow[b]{3}{*}{ Area } & \multirow{3}{*}{$\begin{array}{c}\text { MVS } \\
\text { Sample ID }\end{array}$} & \multirow{2}{*}{\multicolumn{2}{|c|}{ Collection }} & \multirow{3}{*}{$\begin{array}{c}\text { Water } \\
\text { Depth } \\
\text { (ft, Un- } \\
\text { corrected) }\end{array}$} & \multirow{2}{*}{\multicolumn{2}{|c|}{$\begin{array}{c}\text { Coordinates } \\
\text { (dd mm.mmm, NAD 83) }\end{array}$}} & \multirow{3}{*}{$\begin{array}{l}\text { Maximum } \\
\text { Penetration } \\
\text { Depth }(\mathrm{cm}) \\
\end{array}$} & \multirow{3}{*}{$\begin{array}{l}\text { Sampled } \\
\text { Interval } \\
\text { (cm) }\end{array}$} & \multirow[b]{3}{*}{ Comments } \\
\hline & & & & & & & & & \\
\hline & & Date & Time & & Latitude & Longitude & & & \\
\hline Ostrich Bay & MVS- 023 & 08/28/03 & 1535 & 30 & $47^{\circ} 36.084$ & $122^{\circ} 40.853$ & 15 & $0-10$ & $\begin{array}{l}\text { Gray sand, lots of algae and worms; } 2 \text { unsuccessful } \\
\text { attempts on } 08 / 28 / 03\end{array}$ \\
\hline Ostrich Bay & MVS- 024 & 08/28/03 & 1546 & 30 & $47^{\circ} 35.833$ & $122^{\circ} 40.999$ & 11 & $0-10$ & Dark gray silt clay. \\
\hline Ostrich Bay & MVS- 025 & 08/28/03 & 1625 & 26 & $47^{\circ} 35.589$ & $122^{\circ} 40.929$ & 12 & $0-10$ & $\begin{array}{l}\text { Station position moved NW from planned } \\
\text { coordinates for third attempt; root-like mats } \\
\text { (bryozoan or hydroid colonies) on and in sediment, } \\
\text { which was dark gray-brown silt clay. }\end{array}$ \\
\hline Ostrich Bay & MVS- 026 & 08/28/03 & 1651 & 37 & $47^{\circ} 35.332$ & $122^{\circ} 41.001$ & 15 & $0-10$ & Gray-brown silt clay, no odor. \\
\hline Ostrich Bay & MVS- 027 & 08/29/03 & 1007 & 25.0 & $47^{\circ} 35.070$ & $122^{\circ} 41.158$ & 9 & $0-8$ & $\begin{array}{l}\text { Position moved west (inshore) for third attempt. } \\
\text { Dark gray-brown find sand in silt \& clay, few shells, } \\
\text { moderate H2S odor, few worm tubes. }\end{array}$ \\
\hline Ostrich Bay & MVS- 028 & $08 / 29 / 03$ & 1021 & 16.0 & $47^{\circ} 35.066$ & $122^{\circ} 40.839$ & 7 & $0-7$ & $\begin{array}{l}\text { Position moved slightly for second attempt. Dark } \\
\text { gray-brown medium fine sand with clay, slight H2S } \\
\text { odor, lots of biota 9snails, crab, algae, worm tubes. }\end{array}$ \\
\hline Ostrich Bay & MVS- 029 & 08/29/03 & 0926 & 22.4 & $47^{\circ} 34.832$ & $122^{\circ} 40.998$ & 15 & $0-10$ & Gray-brown silt clay, few shells, no odor. \\
\hline Oyster Bay & MVS- 052 & 08/29/03 & 0855 & 17.5 & $47^{\circ} 34.362$ & $122^{\circ} 40.562$ & 15 & $0-10$ & $\begin{array}{l}\text { Dark gray silt clay with some large shells, very } \\
\text { cohesive. Slight H2S odor. }\end{array}$ \\
\hline Dyes Inlet Nondepositional (East) & MVS- 030 & $08 / 29 / 03$ & 1559 & 15 & $47^{\circ} 37.330$ & $122^{\circ} 40.831$ & 6 & $0-5$ & $\begin{array}{l}\text { Gray med-coarse sand and gravel with shells and } \\
\text { shell hash, no odor; } 2 \text { unsuccessful attempts on } \\
08 / 28 / 03\end{array}$ \\
\hline Dyes Inlet Nondepositional (East) & MVS- 031 & $08 / 28 / 03$ & 1350 & 108 & $47^{\circ} 37.062$ & $122^{\circ} 41.301$ & 12 & $0-10$ & Dark gray-brown fine silt, slight H2S odor. \\
\hline Dyes Inlet Nondepositional (East) & MVS- 032 & $08 / 31 / 03$ & 0950 & 24.6 & $47^{\circ} 37.060$ & $122^{\circ} 40.363$ & 4.5 & $0-4$ & $\begin{array}{l}\text { Gray coarse sand and gravel with shells and pebbles, } \\
\text { no odor. }\end{array}$ \\
\hline Dyes Inlet Nondepositional (East) & MVS- 033 & 08/28/03 & 1408 & 62.4 & $47^{\circ} 36.792$ & $122^{\circ} 40.834$ & 15 & $0-10$ & Dark gray fine sand, silt, clay; very cohesive. \\
\hline Dyes Inlet Nondepositional (East) & MVS- 034 & 08/31/03 & 1003 & 63 & $47^{\circ} 36.790$ & $122^{\circ} 39.897$ & 6 & $0-5$ & $\begin{array}{l}\text { Gray medium sand, well-sorted with few shell } \\
\text { pieces, no odor. Right on planned location. }\end{array}$ \\
\hline Dyes Inlet Nondepositional (East) & MVS- 035 & $08 / 28 / 03$ & 1434 & 59 & $47^{\circ} 36.521$ & $122^{\circ} 40.367$ & 6.5 & $0-6$ & Gray medium and coarse sand with shells, pebbles \\
\hline Dyes Inlet Nondepositional (East) & MVS- 036 & 08/31/03 & 0938 & 34.8 & $47^{\circ} 36.256$ & $122^{\circ} 39.751$ & 5 & $0-5$ & $\begin{array}{l}2 \text { unsuccessful attempts on } 08 / 28 / 03,2 \text { more } \\
08 / 31 / 03 \text { before relocated to north side of channel } \\
\text { and sampled at slack current. } 5^{\text {th }} \text { attempt kept, coarse } \\
\text { gray sand, gravel, pebbles, and shell hash. }\end{array}$ \\
\hline Phinney Bay & MVS- 054 & 08/29/03 & 1618 & 29 & $47^{\circ} 35.094$ & $122^{\circ} 39.750$ & 8 & $0-7$ & $\begin{array}{l}\text { Dark gray-brown medium fine sand with clay, few } \\
\text { worms, no odor. }\end{array}$ \\
\hline
\end{tabular}


Table 3. (continued)

\begin{tabular}{|c|c|c|c|c|c|c|c|c|c|}
\hline \multirow[b]{2}{*}{ Area } & \multirow{2}{*}{$\begin{array}{c}\text { MVS } \\
\text { Sample ID }\end{array}$} & \multicolumn{2}{|c|}{ Collection } & \multirow{2}{*}{$\begin{array}{c}\text { Water } \\
\text { Depth } \\
\text { (ft, Un- } \\
\text { corrected) }\end{array}$} & \multicolumn{2}{|c|}{$\begin{array}{c}\text { Coordinates } \\
\text { (dd mm.mmm, NAD 83) }\end{array}$} & \multirow{2}{*}{$\begin{array}{l}\text { Maximum } \\
\text { Penetration } \\
\text { Depth (cm) }\end{array}$} & \multirow{2}{*}{$\begin{array}{l}\text { Sampled } \\
\text { Interval } \\
\text { (cm) }\end{array}$} & \multirow[b]{2}{*}{ Comments } \\
\hline & & Date & Time & & Latitude & Longitude & & & \\
\hline Port Orchard Passage & MVS- 037 & 08/31/03 & 0902 & 32.4 & $47^{\circ} 33.943$ & $122^{\circ} 37.102$ & 2 & $0-2$ & $\begin{array}{l}\text { Kept all material since very difficult area to grab; } \\
\text { Gray coarse sand, gravel, mostly pebbles, some } \\
\text { shells, no odor. }\end{array}$ \\
\hline Port Orchard Passage & MVS- 038 & 08/30/03 & 1342 & 42.0 & $47^{\circ} 34.296$ & $122^{\circ} 36.139$ & 3 & $0-2$ & $\begin{array}{l}\text { Gray sand; } 4 \text { unsuccessful attempts before retaining } \\
\text { a few cm sand. }\end{array}$ \\
\hline Port Orchard Passage & MVS- 039 & 08/30/03 & 1236 & 163 & $47^{\circ} 34.473$ & $122^{\circ} 35.655$ & 4 & $0-3$ & $\begin{array}{l}\text { Coarse gray sand and gravel with shell hash, } \\
\text { unsorted, many shells \& pieces of shells, no odor. }\end{array}$ \\
\hline Port Orchard Passage & MVS- 040 & 08/30/03 & 1458 & 70.0 & $47^{\circ} 33.437$ & $122^{\circ} 37.215$ & 12 & $0-10$ & $\begin{array}{l}\text { Gray brown medium-fine sand, silt, clay, worms, } \\
\text { few hydroids. }\end{array}$ \\
\hline Port Orchard Passage & MVS- 041 & 08/31/03 & 0847 & 110 & $47^{\circ} 33.613$ & $122^{\circ} 36.707$ & 3 & $0-2$ & $\begin{array}{l}\text { Sample is composite of three grabs, each with } 1-3 \\
\text { cm retained. Position varied }<10 \text { m between grabs. } \\
\text { Coarse gray sand, gravel, cobbles, and shells. }\end{array}$ \\
\hline Port Orchard Passage & MVS- 042 & 08/30/03 & 1355 & 84.6 & $47^{\circ} 33.789$ & $122^{\circ} 36.224$ & 8 & $0-7$ & $\begin{array}{l}\text { Gray-brown fine sand and silt, diatoms on surface, } \\
\text { worms \& tubes, no odor. }\end{array}$ \\
\hline Port Orchard Passage & MVS- 043 & 08/30/03 & 1307 & 73.6 & $47^{\circ} 33.966$ & $122^{\circ} 35.743$ & 7 & $0-6$ & $\begin{array}{l}\text { Gray medium fine sand, no odor. Right on planned } \\
\text { position! }\end{array}$ \\
\hline Port Orchard Passage & MVS- 044 & $08 / 30 / 03$ & 1251 & 27.0 & $47^{\circ} 34.143$ & $122^{\circ} 35.261$ & 8.5 & $0-8$ & Gray fine sand with silt \& clay. \\
\hline Port Orchard Passage & MVS- 045 & 08/30/03 & 1424 & 55.0 & $47^{\circ} 33.107$ & $122^{\circ} 36.802$ & 8 & $0-7$ & Gray brown silt and clay, many worms. \\
\hline Port Orchard Passage & MVS- 046 & 08/30/03 & 1411 & 30.4 & $47^{\circ} 33.284$ & $122^{\circ} 36.318$ & 8 & $0-6$ & $\begin{array}{l}\text { Gray-brown fine sand and silt, diatoms on surface, } \\
\text { worms, no odor. }\end{array}$ \\
\hline Port Orchard Passage & MVS- 047 & 08/30/03 & 1012 & 112 & $47^{\circ} 37.416$ & $122^{\circ} 35.053$ & 12 & $0-10$ & $\begin{array}{l}\text { Dark gray silt clay, very cohesive, worms present, no } \\
\text { odor. }\end{array}$ \\
\hline Port Orchard Passage & MVS- 048 & 08/30/03 & 1046 & 49 & $47^{\circ} 36.133$ & $122^{\circ} 35.645$ & 12 & $0-10$ & $\begin{array}{l}\text { Gray fine cohesive sand with silt \& clay, many large } \\
\text { worms, algae. }\end{array}$ \\
\hline Port Orchard Passage & MVS- 049 & 08/30/03 & 1029 & 72 & $47^{\circ} 36.526$ & $122^{\circ} 34.694$ & 12.5 & $0-10$ & $\begin{array}{l}\text { Dark gray silt clay, some fine sand, slight H2S odor, } \\
\text { no organisms. }\end{array}$ \\
\hline Port Orchard Passage & MVS- 051 & 08/30/03 & 1145 & 211 & $47^{\circ} 34.900$ & $122^{\circ} 34.975$ & 0 & 0 & $\begin{array}{l}\text { NO SAMPLE POSSIBLE--tried } 2 \text { locations, large } \\
\text { rocks only. }\end{array}$ \\
\hline Port Orchard Passage & MVS- 053 & 08/30/03 & 1120 & 152 & $47^{\circ} 35.150$ & $122^{\circ} 35.199$ & 0 & 0 & $\begin{array}{l}\text { NO SAMPLE POSSIBLE--tried } 2 \text { locations, large } \\
\text { rocks and shells only. }\end{array}$ \\
\hline
\end{tabular}


Table 3. (continued)

\begin{tabular}{|c|c|c|c|c|c|c|c|c|c|}
\hline \multirow[b]{2}{*}{ Area } & \multirow{2}{*}{$\begin{array}{c}\text { MVS } \\
\text { Sample ID }\end{array}$} & \multicolumn{2}{|c|}{ Collection } & \multirow{2}{*}{$\begin{array}{c}\text { Water } \\
\text { Depth } \\
\text { (ft, Un- } \\
\text { corrected) }\end{array}$} & \multicolumn{2}{|c|}{$\begin{array}{c}\text { Coordinates } \\
\text { (dd mm.mmm, NAD 83) }\end{array}$} & \multirow{2}{*}{$\begin{array}{l}\text { Maximum } \\
\text { Penetration } \\
\text { Depth }(\mathrm{cm})\end{array}$} & \multirow{2}{*}{$\begin{array}{l}\text { Sampled } \\
\text { Interval } \\
(\mathbf{c m})\end{array}$} & \multirow[b]{2}{*}{ Comments } \\
\hline & & Date & Time & & Latitude & Longitude & & & \\
\hline $\begin{array}{l}\text { Port Orchard Passage, Fletcher Bay } \\
\text { entrance }\end{array}$ & MVS- 058 & 08/31/03 & 1117 & 24.6 & $47^{\circ} 38.640$ & $122^{\circ} 34.928$ & 4.5 & $0-4$ & $\begin{array}{l}\text { Gray coarse sand and gravel, shell hash, no odor. } \\
\text { Sample collected to WNW of Fletcher Bay entrance } \\
\text { after 5-6 attempts to collect closer to shore and bay } \\
\text { mouth. }\end{array}$ \\
\hline Port Orchard Passage, Brownsville & MVS- 059 & 08/31/03 & 1140 & 21.5 & $47^{\circ} 38.977$ & $122^{\circ} 36.757$ & 15 & $0-10$ & $\begin{array}{l}\text { Dark gray silt and clay, very soft, moderate } \mathrm{H} 2 \mathrm{~S} \\
\text { odor. }\end{array}$ \\
\hline Rich Passage, north & MVS- 050 & 08/30/03 & 0946 & 6.4 & $47^{\circ} 36.109$ & $122^{\circ} 32.950$ & 3 & $0-3$ & \begin{tabular}{|l|} 
Positioned in shallow water as close to planned as \\
possible; Gray medium sand, no odor, clean \& dry.
\end{tabular} \\
\hline Rich Passage, NW of Orchard Rocks & MVS- 057 & 08/30/03 & 0929 & 24 & $47^{\circ} 35.217$ & $122^{\circ} 31.915$ & 3 & $0-3$ & $\begin{array}{l}\text { Moved } \mathrm{N} \text { after } 2 \text { attempts in rocks; successful } \\
\text { sample collected just SE of small boat ramp. } \\
\text { Medium gray sand with shells, no odor. }\end{array}$ \\
\hline $\begin{array}{l}\text { Clam Bay north, near Manchester } \\
\text { Pier }\end{array}$ & MVS- 055 & 08/30/03 & 0843 & 13 & $47^{\circ} 34.308$ & $122^{\circ} 32.770$ & 7 & $0-6$ & $\begin{array}{l}\text { Kept sediment from 5th attempt only. Gray medium } \\
\text { fine sand and silt, no odor, eelgrass growing in sand. }\end{array}$ \\
\hline $\begin{array}{l}\text { Clam Bay South, off mouth of Little } \\
\text { Clam Bay }\end{array}$ & MVS- 056 & 08/30/03 & 0859 & 10.8 & $47^{\circ} 34.125$ & $122^{\circ} 32.800$ & 7 & $0-6$ & $\begin{array}{l}\text { Gray medium fine sand and silt, no odor; green algae } \\
\text { but no eelgrass. }\end{array}$ \\
\hline
\end{tabular}




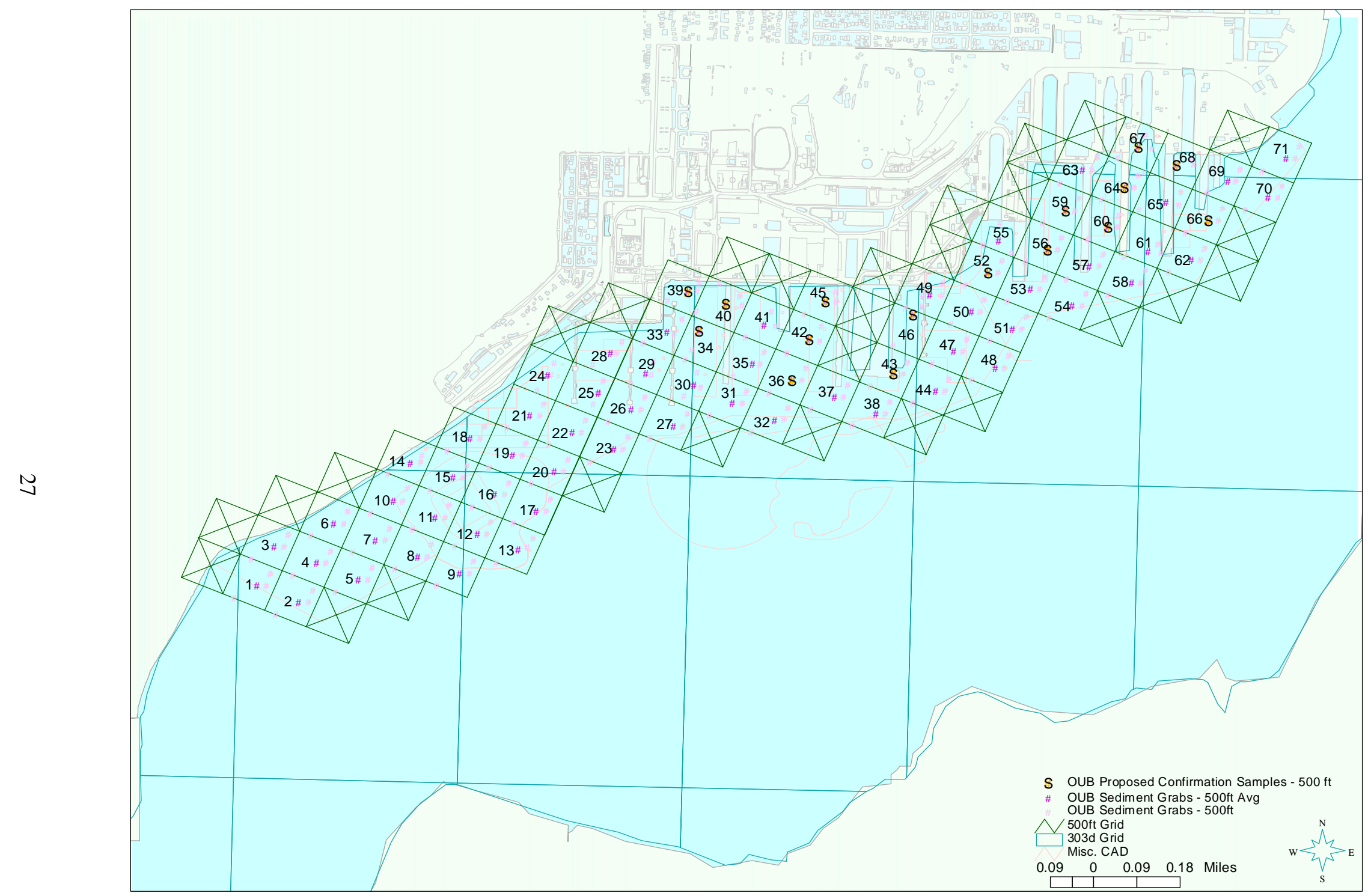

Figure 14. OU B Marine monitoring stations in 500-ft grid within OU B Marine boundary (OUBM) 


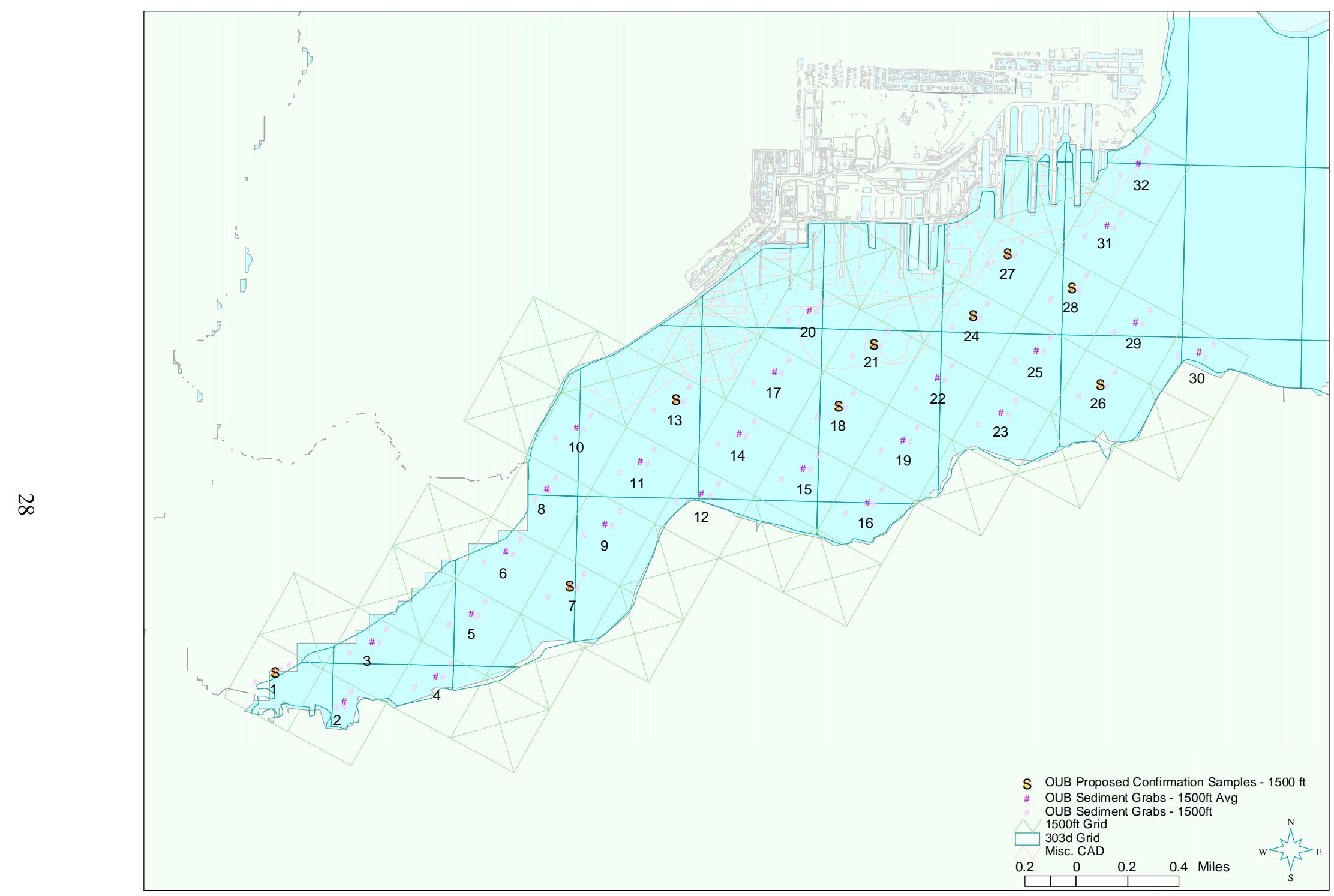

Figure 15. OU B Marine monitoring stations in 1500-ft grid outside OU B Marine boundary (OOUB) 
Table 4. OU B Marine Monitoring Samples Received for Metals Verification Study

\begin{tabular}{|c|c|c|c|c|}
\hline \multirow[b]{2}{*}{ OU B Grid Cell } & \multirow[b]{2}{*}{ 303d Segment(s) } & \multicolumn{3}{|c|}{$\begin{array}{c}\text { OU B Marine Monitoring Sample } \\
\text { Collection Information }\end{array}$} \\
\hline & & $\begin{array}{l}\text { Composite } \\
\text { Sample ID }\end{array}$ & $\begin{array}{l}\text { Collection } \\
\text { Date }\end{array}$ & $\begin{array}{l}\text { Collection } \\
\text { Time }\end{array}$ \\
\hline \multicolumn{5}{|c|}{ Inside OU B Marine (OUBM) } \\
\hline OUBM-G 01 & $47122 \quad$ F6E6 & BNC 63181 & $10 / 13 / 2003$ & 1020 \\
\hline OUBM-G 02 & $47122 \quad$ F6E6 & BNC 63182 & $10 / 13 / 2003$ & 1045 \\
\hline OUBM-G 03 & 47122 F6E6 & BNC 63183 & $10 / 13 / 2003$ & 1120 \\
\hline OUBM-G 04 & 47122 F6E6 & BNC 63184 & $10 / 13 / 2003$ & 1150 \\
\hline OUBM-G 05 & 47122 & BNC 63185 & $10 / 13 / 2003$ & 1215 \\
\hline OUBM-G 06 & 47122 F6E6 & BNC 63186 & $10 / 13 / 2003$ & 1330 \\
\hline OUBM-G 07 & 47122 & BNC 63188 & $10 / 13 / 2003$ & 1410 \\
\hline OUBM-G 08 & $47122 \quad$ F6E6 & BNC 63189 & $10 / 13 / 2003$ & 1440 \\
\hline OUBM-G 09 & F6E6, F6E5 & BNC 63190 & $10 / 13 / 2003$ & 1510 \\
\hline OUBM-G 10 & F6E6 & BNC 63191 & $10 / 13 / 2003$ & 1540 \\
\hline OUBM-G 11 & 47122 F6E6 & BNC 63193 & $10 / 20 / 2003$ & 1030 \\
\hline OUBM-G 12 & F6E5, F6E6 & BNC 63194 & $10 / 20 / 2003$ & 1100 \\
\hline OUBM-G 13 & $47122 \quad$ F6E5 & BNC 63195 & $10 / 20 / 2003$ & 1135 \\
\hline OUBM-G 14 & F6F6, F6F5 & BNC 63196 & $10 / 20 / 2003$ & 1315 \\
\hline OUBM-G 15 & F6E6, F6F6 & BNC 63197 & $10 / 20 / 2003$ & 1355 \\
\hline OUBM-G 16 & $47122 \quad$ F6E5 & BNC 63198 & $10 / 20 / 2003$ & 1430 \\
\hline OUBM-G 17 & 47122 & BNC 63200 & $10 / 20 / 2003$ & 1515 \\
\hline OUBM-G 18 & F6F6, F6F5 & BNC 63221 & $10 / 20 / 2003$ & 1545 \\
\hline OUBM-G 19 & F6F5 & BNC 63228 & $10 / 22 / 2003$ & 1640 \\
\hline OUBM-G 20 & F6F5, F6F6 & BNC 63253 & $10 / 27 / 2003$ & 0920 \\
\hline OUBM-G 21 & F6F5 & BNC 63254 & $10 / 27 / 2003$ & 0945 \\
\hline OUBM-G 22 & $47122 \quad$ F6F5 & BNC 63255 & $10 / 27 / 2003$ & 1010 \\
\hline OUBM-G 23 & $47122 \quad$ F6F5 & BNC 63256 & $10 / 27 / 2003$ & 1035 \\
\hline OUBM-G 24 & 47122 & BNC 63267 & $10 / 28 / 2003$ & 0950 \\
\hline OUBM-G 25 & $47122 \quad$ F6F5 & BNC 63257 & $10 / 27 / 2003$ & 1125 \\
\hline OUBM-G 26 & 47122 & BNC 63259 & $10 / 27 / 2003$ & 1155 \\
\hline OUBM-G 27 & 47122 & BNC 63240 & $10 / 23 / 2003$ & 1615 \\
\hline OUBM-G 28 & 47122 & BNC 63262 & $10 / 27 / 2003$ & 1420 \\
\hline OUBM-G 29 & 47122 & BNC 63261 & $10 / 27 / 2003$ & 1310 \\
\hline OUBM-G 30 & $47122 \quad$ F6F5, F6F4 & BNC 63230 & $10 / 23 / 2003$ & 0915 \\
\hline OUBM-G 31 & 47122 & BNC 63233 & $10 / 23 / 2003$ & 1145 \\
\hline OUBM-G 32 & $\begin{array}{ll}47122 & \text { F6F4 } \\
\end{array}$ & BNC 63251 & $10 / 24 / 2003$ & 1550 \\
\hline OUBM-G 33 & 47122 & BNC 63260 & $10 / 27 / 2003$ & 1445 \\
\hline OUBM-G 34 & F6F5, F6F4 & BNC 63232 & $10 / 23 / 2003$ & 1120 \\
\hline OUBM-G 35 & F6F4 & BNC 63227 & $10 / 22 / 2003$ & 1600 \\
\hline OUBM-G 36 & $47122 \quad$ F6F4 & BNC 63235 & $10 / 23 / 2003$ & 1245 \\
\hline OUBM-G 37 & $47122 \quad \mathrm{~F} 6 \mathrm{~F} 4$ & BNC 63239 & $10 / 23 / 2003$ & 1545 \\
\hline OUBM-G & 47122 & BNC 63248 & $10 / 24 / 2003$ & 1430 \\
\hline OUBM-G 39 & 47122 & BNC 63231 & $10 / 23 / 2003$ & 1015 \\
\hline
\end{tabular}


Table 4. (continued)

\begin{tabular}{|c|c|c|c|c|}
\hline \multirow[b]{2}{*}{ OU B Grid Cell } & \multirow[b]{2}{*}{ 303d Segment(s) } & \multicolumn{3}{|c|}{$\begin{array}{c}\text { OU B Marine Monitoring Sample } \\
\text { Collection Information }\end{array}$} \\
\hline & & $\begin{array}{l}\text { Composite } \\
\text { Sample ID }\end{array}$ & $\begin{array}{c}\text { Collection } \\
\text { Date }\end{array}$ & $\begin{array}{c}\text { Collection } \\
\text { Time }\end{array}$ \\
\hline \multicolumn{5}{|c|}{ Inside OU B Marine (OUBM) continued } \\
\hline OUBM-G 40 & $47122 \quad$ F6F4 & BNC 63226 & $10 / 22 / 2003$ & 1500 \\
\hline OUBM-G 41 & $47122 \quad$ F6F4 & BNC 63225 & $10 / 22 / 2003$ & 1340 \\
\hline OUBM-G 42 & $47122 \quad \mathrm{~F} 6 \mathrm{~F} 4$ & BNC 63224 & $10 / 22 / 2003$ & 1230 \\
\hline OUBM-G 43 & $47122 \quad$ F6F4 & BNC 63236 & $10 / 23 / 2003$ & 1330 \\
\hline OUBM-G & F6F3, F6F4 & BNC 63242 & $10 / 24 / 2003$ & 0910 \\
\hline OUBM-G 45 & $47122 \quad \mathrm{~F} 6 \mathrm{~F} 4$ & BNC 63223 & $10 / 22 / 2003$ & 1130 \\
\hline OUBM-G 46 & $47122 \quad \mathrm{~F} 6 \mathrm{~F} 4, \mathrm{~F} 6 \mathrm{~F} 3$ & BNC 63238 & $10 / 23 / 2003$ & 1510 \\
\hline OUBM-G 47 & $47122 \quad$ F6F3 & BNC 63243 & $10 / 24 / 2003$ & 0945 \\
\hline OUBM-G 48 & $47122 \quad$ F6F3 & BNC 63249 & $10 / 24 / 2003$ & 1505 \\
\hline OUBM-G 49 & $47122 \quad$ F6F4, F6F3 & BNC 63237 & $10 / 23 / 2003$ & 1410 \\
\hline OUBM-G 50 & $47122 \quad$ F6F3 & BNC 63244 & $10 / 24 / 2003$ & 1025 \\
\hline OUBM-G & 47122 F6F3 & BNC 63250 & $10 / 24 / 2003$ & 1525 \\
\hline OUBM-G 52 & 47122 F6F3 & BNC 63245 & $10 / 24 / 2003$ & 1050 \\
\hline OUBM-G 53 & $47122 \quad$ F6F3 & BNC 63165 & $10 / 3 / 2003$ & 1330 \\
\hline OUBM-G 54 & $47122 \quad$ F6F3 & BNC 63167 & $10 / 3 / 2003$ & 1545 \\
\hline OUBM-G 55 & $47122 \quad$ F6F3 & BNC 63247 & $10 / 24 / 2003$ & 1245 \\
\hline OUBM-G 56 & $47122 \quad$ F6F3 & BNC 63163 & $10 / 3 / 2003$ & 1210 \\
\hline OUBM-G 57 & $47122 \quad$ F6F3 & BNC 63162 & $10 / 3 / 2003$ & 1130 \\
\hline OUBM-G & $47122 \quad$ F6F3 & BNC 63153 & $10 / 2 / 2003$ & 1015 \\
\hline OUBM-G 59 & 47122 F6F3 & BNC 63161 & $10 / 3 / 2003$ & 1050 \\
\hline OUBM-G 60 & 47122 F6F3 & BNC 63166 & $10 / 3 / 2003$ & 1450 \\
\hline OUBM-G 61 & $47122 \quad$ F6F2, F6F3 & BNC 63154 & $10 / 2 / 2003$ & 1215 \\
\hline OUBM-G 62 & $47122 \quad \mathrm{~F} 6 \mathrm{~F} 2$ & BNC 63155 & $10 / 2 / 2003$ & 1350 \\
\hline OUBM-G 63 & $47122 \quad$ F6F3 & BNC 63160 & $10 / 3 / 2003$ & 1010 \\
\hline OUBM-G 64 & $47122 \quad$ F6F3 & BNC 63156 & $10 / 2 / 2003$ & 1430 \\
\hline OUBM-G 65 & $47122 \quad$ F6F2, F6F3 & BNC 63263 & $10 / 27 / 2003$ & 1530 \\
\hline OUBM-G 66 & $47122 \quad$ F6F2 & BNC 63157 & $10 / 2 / 2003$ & 1610 \\
\hline OUBM-G 67 & $47122 \quad$ F6G3? & BNC 63159 & $10 / 3 / 2003$ & 0930 \\
\hline OUBM-G 68 & $47122 \quad$ F6G2 & BNC 63264 & $10 / 27 / 2003$ & 1615 \\
\hline OUBM-G 69 & $47122 \quad$ F6F2, F6G2 & BNC 63265 & $10 / 27 / 2003$ & 1710 \\
\hline OUBM-G 70 & $47122 \quad$ F6F2 & BNC 63179 & $10 / 10 / 2003$ & 1415 \\
\hline OUBM-G 71 & $47122 \quad$ F6G2 & BNC 63178 & $10 / 10 / 2003$ & 1325 \\
\hline \multicolumn{5}{|c|}{ Outside OU B Marine (OOUB) } \\
\hline OOUB-G 01 & $47122 \quad$ F6C9 & BNC 63304 & 10/30/2003 & 1700 \\
\hline OOUB-G & F6C8, F6C9 & BNC 63280 & $10 / 29 / 2003$ & 0940 \\
\hline OOUB-G 03 & $47122 \quad$ F6C8, F6D8 & BNC 63281 & $10 / 29 / 2003$ & 1015 \\
\hline OOUB-G & F6C8, F6D8 & BNC 63282 & $10 / 29 / 2003$ & 1040 \\
\hline OOUB-G 05 & $47122 \quad$ F6D8, F6D7 & BNC 63283 & $10 / 29 / 2003$ & 1115 \\
\hline OOUB-G & 47122 & BNC 63284 & $10 / 29 / 2003$ & 1145 \\
\hline
\end{tabular}


Table 4. (continued)

\begin{tabular}{|c|c|c|c|c|}
\hline \multirow[b]{2}{*}{ OU B Grid Cell } & \multirow[b]{2}{*}{ 303d Segment(s) } & \multicolumn{3}{|c|}{$\begin{array}{c}\text { OU B Marine Monitoring Sample } \\
\text { Collection Information }\end{array}$} \\
\hline & & $\begin{array}{l}\text { Composite } \\
\text { Sample ID }\end{array}$ & $\begin{array}{c}\text { Collection } \\
\text { Date }\end{array}$ & $\begin{array}{c}\text { Collection } \\
\text { Time }\end{array}$ \\
\hline \multicolumn{5}{|c|}{ Outside OU B Marine (OOUB), continued } \\
\hline OOUB-G 07 & $47122 \quad$ F6D7 & BNC 63302 & $10 / 30 / 2003$ & 1455 \\
\hline OOUB-G 08 & $\begin{array}{ll}47122 & \text { F6E7 } \\
\end{array}$ & BNC 63301 & $10 / 30 / 2003$ & 1415 \\
\hline OOUB-G 09 & $47122 \quad$ F6D7, F6D6 & BNC 63300 & $10 / 30 / 2003$ & 1355 \\
\hline OOUB-G 10 & $47122 \quad$ F6E7 & BNC 63299 & $10 / 30 / 2003$ & 1320 \\
\hline OOUB-G 11 & 47122 F6E6 & BNC 63298 & $10 / 30 / 2003$ & 1250 \\
\hline OOUB-G 13 & 47122 F6E6 & BNC 63297 & $10 / 30 / 2003$ & 1145 \\
\hline OOUB-G 14 & 47122 F6E5 & BNC 63295 & $10 / 30 / 2003$ & 1115 \\
\hline OOUB-G 15 & $47122 \quad$ F6E5 & BNC 63268 & $10 / 28 / 2003$ & 1045 \\
\hline OOUB-G 17 & $47122 \quad$ F6E5 & BNC 63294 & $10 / 30 / 2003$ & 1045 \\
\hline OOUB-G 18 & $47122 \quad$ F6E4, F6E5 & BNC 63293 & $10 / 30 / 2003$ & 1015 \\
\hline OOUB-G 19 & $47122 \quad$ F6E4 & BNC 63270 & $10 / 28 / 2003$ & 1145 \\
\hline OOUB-G 20 & $47122 \quad$ F6F5 & BNC 63292 & $10 / 30 / 2003$ & 0945 \\
\hline OOUB-G 21 & $47122 \quad$ F6E4 & BNC 63291 & $10 / 30 / 2003$ & 0915 \\
\hline OOUB-G 22 & $47122 \quad$ F6E4 & BNC 63277 & $10 / 28 / 2003$ & 1625 \\
\hline OOUB-G 23 & 47122 F6E3 & BNC 63271 & $10 / 28 / 2003$ & 1215 \\
\hline OOUB-G 24 & 47122 F6F3 & BNC 63276 & $10 / 28 / 2003$ & 1540 \\
\hline OOUB-G 25 & $47122 \quad$ F6E3 & BNC 63272 & $10 / 28 / 2003$ & 1345 \\
\hline OOUB-G 26 & 47122 F6E2 & BNC 63287 & $10 / 29 / 2003$ & 1420 \\
\hline $\begin{array}{ll}\text { OOUB-G } \quad 27 \\
\end{array}$ & $\begin{array}{ll}47122 & \text { F6F3 } \\
\end{array}$ & BNC 63275 & $10 / 28 / 2003$ & 1510 \\
\hline OOUB-G & $47122 \quad$ F6F2, F6F3 & BNC 63274 & $10 / 28 / 2003$ & 1445 \\
\hline OOUB-G 29 & $\begin{array}{ll}47122 & \text { F6F2 } \\
\end{array}$ & BNC 63273 & $10 / 28 / 2003$ & 1410 \\
\hline OOUB-G 31 & $47122 \quad \mathrm{~F} 6 \mathrm{~F} 2$ & BNC 63285 & $10 / 29 / 2003$ & 1315 \\
\hline OOUB-G 32 & $47122 \quad$ F6F2 & BNC 63286 & $10 / 29 / 2003$ & 1545 \\
\hline
\end{tabular}


Table 5. XRF Screening Results

\begin{tabular}{|c|c|c|c|c|c|c|}
\hline \multirow{2}{*}{ Location Description } & \multirow{2}{*}{$\begin{array}{c}\text { Station or OUB } \\
\text { Grid Cell }\end{array}$} & \multicolumn{5}{|c|}{ XRF Screening Concentration (mg/kg) } \\
\hline & & \multirow{4}{*}{$\begin{array}{c}\mathrm{Cu} \\
390 \\
351 \\
390\end{array}$} & \multirow{4}{*}{$\begin{array}{c}\mathrm{Pb} \\
450 \\
405 \\
530\end{array}$} & \multirow{4}{*}{$\begin{array}{r}\mathrm{Zn} \\
410 \\
369 \\
960 \\
\end{array}$} & \multirow{4}{*}{$\begin{array}{l}\text { Cd } \\
5.1 \\
4.6 \\
6.7\end{array}$} & \multirow{4}{*}{$\begin{array}{l}\text { Ag } \\
6.1 \\
5.5 \\
6.1\end{array}$} \\
\hline SC & & & & & & \\
\hline $90 \% \mathrm{SC}$ & & & & & & \\
\hline MCL & & & & & & \\
\hline Dyes Depositional Zone & MVS-001 & 34 & 17 & 58 & $1.2 \mathrm{~J}^{\mathrm{a}}$ & $0.1 \mathrm{U}^{\mathrm{b}}$ \\
\hline Dyes Depositional Zone & MVS-002 & $18 \mathrm{~J}$ & $9 \mathrm{~J}$ & 38 & $2.3 \mathrm{~J}$ & $0.3 \mathrm{U}$ \\
\hline Dyes Depositional Zone & MVS-003 & 46 & 30 & 84 & $0.5 \mathrm{U}$ & $0.3 \mathrm{U}$ \\
\hline Dyes Depositional Zone & MVS-004 & 63 & 41 & 98 & $\mathrm{ND}^{\mathrm{c}}$ & $0.9 \mathrm{U}$ \\
\hline Dyes Depositional Zone & MVS-005 & 56 & 38 & 91 & $0.6 \mathrm{U}$ & $1.4 \mathrm{~J}$ \\
\hline Dyes Depositional Zone & MVS-006 & $25 \mathrm{~J}$ & 16 & 52 & $0.8 \mathrm{U}$ & $0.4 \mathrm{U}$ \\
\hline Dyes Depositional Zone & MVS-007 & 57 & 39 & 97 & $0.6 \mathrm{U}$ & $0.7 \mathrm{U}$ \\
\hline Dyes Depositional Zone & MVS-008 & 41 & 24 & 85 & ND & $0.9 \mathrm{U}$ \\
\hline Dyes Depositional Zone & MVS-009 & 47 & 36 & 85 & $0.7 \mathrm{U}$ & $1.8 \mathrm{~J}$ \\
\hline Dyes Depositional Zone & MVS-010 & 47 & 31 & 91 & $0.5 \mathrm{U}$ & $0.2 \mathrm{U}$ \\
\hline Dyes Depositional Zone & MVS-011 & 43 & 37 & 95 & $0.5 \mathrm{U}$ & $0.8 \mathrm{U}$ \\
\hline Dyes Depositional Zone & MVS-012 & 57 & 38 & 94 & $0.4 \mathrm{U}$ & $0.6 \mathrm{U}$ \\
\hline \begin{tabular}{|l|} 
Dyes Depositional Zone \\
\end{tabular} & MVS-013 & 43 & 30 & 86 & $0.7 \mathrm{U}$ & ND \\
\hline Dyes Depositional Zone & MVS-014 & 37 & 15 & 62 & ND & ND \\
\hline Dyes Depositional Zone & MVS-015 & $28 \mathrm{~J}$ & $9 \mathrm{~J}$ & 59 & $0.0 \mathrm{U}$ & $0.4 \mathrm{U}$ \\
\hline Dyes Depositional Zone & MVS-016 & 34 & $14 \mathrm{~J}$ & 65 & $0.7 \mathrm{U}$ & $0.1 \mathrm{U}$ \\
\hline Dyes Depositional Zone & MVS-017 & 45 & 27 & 79 & $0.3 \mathrm{U}$ & $0.3 \mathrm{U}$ \\
\hline Dyes Depositional Zone & MVS-018 & 44 & 36 & 84 & $1.5 \mathrm{~J}$ & $0.9 \mathrm{U}$ \\
\hline Dyes Depositional Zone & MVS-019 & 34 & $13 \mathrm{~J}$ & 45 & $0.6 \mathrm{U}$ & $0.1 \mathrm{U}$ \\
\hline Dyes Depositional Zone & MVS-020 & 45 & 32 & 77 & ND & ND \\
\hline Dyes Depositional Zone & MVS-021 & 46 & 26 & 72 & $1.0 \mathrm{~J}$ & $1.3 \mathrm{~J}$ \\
\hline Dyes Depositional Zone & MVS-022 & 53 & 32 & 89 & $1.3 \mathrm{~J}$ & ND \\
\hline Dyes Nondepositional Zone & MVS-030 & $17 \mathrm{~J}$ & $8 \mathrm{~J}$ & 31 & $0.8 \mathrm{U}$ & ND \\
\hline Dyes Nondepositional Zone & MVS-031 & 29 & $14 \mathrm{~J}$ & 49 & $1.3 \mathrm{~J}$ & ND \\
\hline Dyes Nondepositional Zone & MVS-032 & $16 \mathrm{~J}$ & $8 \mathrm{~J}$ & 33 & ND & ND \\
\hline Dyes Nondepositional Zone & MVS-033 & 29 & 22 & 53 & ND & $1.3 \mathrm{~J}$ \\
\hline Dyes Nondepositional Zone & MVS-034 & $16 \mathrm{~J}$ & $7 \mathrm{~J}$ & 29 & ND & $0.1 \mathrm{U}$ \\
\hline Dyes Nondepositional Zone & MVS-035 & $16 \mathrm{~J}$ & $7 \mathrm{~J}$ & 37 & $0.8 \mathrm{U}$ & ND \\
\hline Dyes Nondepositional Zone & MVS-036 & $18 \mathrm{~J}$ & $10 \mathrm{~J}$ & 33 & ND & ND \\
\hline Ostrich Bay & MVS-023 & 55 & 36 & 93 & $0.2 \mathrm{U}$ & $0.5 \mathrm{U}$ \\
\hline Ostrich Bay & MVS-024 & 49 & 26 & 77 & ND & $0.3 \mathrm{U}$ \\
\hline Ostrich Bay & MVS-025 & 44 & 28 & 71 & $0.7 \mathrm{U}$ & $0.5 \mathrm{U}$ \\
\hline Ostrich Bay & MVS-026 & 47 & 30 & 73 & $0.4 \mathrm{U}$ & $0.5 \mathrm{U}$ \\
\hline Ostrich Bay & MVS-027 & 50 & 30 & 77 & $0.8 \mathrm{U}$ & $0.3 \mathrm{U}$ \\
\hline Ostrich Bay & MVS-028 & $22 \mathrm{~J}$ & $12 \mathrm{~J}$ & 46 & $0.7 \mathrm{U}$ & ND \\
\hline Ostrich Bay & MVS-029 & 48 & 31 & 91 & $2.2 \mathrm{~J}$ & ND \\
\hline Oyster Bay & MVS-052 & 37 & 28 & 78 & $2.3 \mathrm{~J}$ & $0.4 \mathrm{U}$ \\
\hline Phinney Bay & MVS-054 & $22 \mathrm{~J}$ & $12 \mathrm{~J}$ & 46 & $1.3 \mathrm{~J}$ & $1.8 \mathrm{~J}$ \\
\hline
\end{tabular}


Table 5. (continued)

\begin{tabular}{|c|c|c|c|c|c|c|}
\hline \multirow{2}{*}{ Location Description } & \multirow{2}{*}{$\begin{array}{c}\text { Station or OUB } \\
\text { Grid Cell }\end{array}$} & \multicolumn{5}{|c|}{ XRF Screening Concentration (mg/kg) } \\
\hline & & \multirow{4}{*}{$\begin{array}{c}\mathrm{Cu} \\
390 \\
351 \\
390\end{array}$} & \multirow{4}{*}{$\begin{array}{r}\mathrm{Pb} \\
450 \\
405 \\
530\end{array}$} & \multirow{4}{*}{$\begin{array}{c}\mathrm{Zn} \\
410 \\
369 \\
960\end{array}$} & \multirow{4}{*}{$\begin{array}{l}\text { Cd } \\
5.1 \\
4.6 \\
6.7\end{array}$} & \multirow{4}{*}{$\begin{array}{l}\text { Ag } \\
6.1 \\
5.5 \\
6.1 \\
\end{array}$} \\
\hline SQS & & & & & & \\
\hline $90 \%$ SQS & & & & & & \\
\hline MCUL & & & & & & \\
\hline Port Orchard Passage & MVS-037 & $21 \mathrm{~J}$ & $7 \mathrm{~J}$ & 43 & $1.6 \mathrm{~J}$ & ND \\
\hline Port Orchard Passage & MVS-038 & $22 \mathrm{~J}$ & $6 \mathrm{~J}$ & 38 & $0.6 \mathrm{U}$ & ND \\
\hline Port Orchard Passage & MVS-039 & $21 \mathrm{~J}$ & $11 \mathrm{~J}$ & 34 & $0.4 \mathrm{U}$ & $1.0 \mathrm{~J}$ \\
\hline Port Orchard Passage & MVS-040 & 36 & 18 & 70 & $0.7 \mathrm{U}$ & ND \\
\hline Port Orchard Passage & MVS-041 & 45 & 21 & 68 & ND & $\mathrm{ND}$ \\
\hline Port Orchard Passage & MVS-042 & $25 \mathrm{~J}$ & 18 & 52 & $0.7 \mathrm{U}$ & $0.3 \mathrm{U}$ \\
\hline Port Orchard Passage & MVS-043 & $13 \mathrm{~J}$ & $11 \mathrm{~J}$ & 33 & $1.6 \mathrm{~J}$ & $2.3 \mathrm{~J}$ \\
\hline Port Orchard Passage & MVS-044 & $18 \mathrm{~J}$ & $9 \mathrm{~J}$ & 29 & ND & $0.0 \mathrm{U}$ \\
\hline Port Orchard Passage & MVS-045 & 35 & 23 & 62 & $0.5 \mathrm{U}$ & $0.2 \mathrm{U}$ \\
\hline Port Orchard Passage & MVS-046 & 30 & 18 & 44 & ND & $\mathrm{ND}$ \\
\hline Port Orchard Passage (Ref) & MVS-047 & 55 & 22 & 72 & $1.5 \mathrm{~J}$ & $0.4 \mathrm{U}$ \\
\hline Port Orchard Passage (Ref) & MVS-048 & $25 \mathrm{~J}$ & $14 \mathrm{~J}$ & 43 & $0.5 \mathrm{U}$ & $1.7 \mathrm{~J}$ \\
\hline Port Orchard Passage (Ref) & MVS-049 & 39 & 19 & 72 & $1.1 \mathrm{~J}$ & $1.2 \mathrm{~J}$ \\
\hline Rich Passage, north & MVS-050 & $9 \mathrm{~J}$ & $9 \mathrm{~J}$ & $24 \mathrm{~J}$ & $0.4 \mathrm{U}$ & $0.5 \mathrm{U}$ \\
\hline $\begin{array}{l}\text { Rich Passage, NW of Orchard } \\
\text { Rocks }\end{array}$ & MVS-057 & $21 \mathrm{~J}$ & $10 \mathrm{~J}$ & 38 & $0.3 \mathrm{U}$ & $0.7 \mathrm{U}$ \\
\hline $\begin{array}{l}\text { Clam Bay north, near } \\
\text { Manchester Pier }\end{array}$ & MVS-055 & $13 \mathrm{~J}$ & $4 \mathrm{~J}$ & $25 \mathrm{~J}$ & $1.6 \mathrm{~J}$ & $0.4 \mathrm{U}$ \\
\hline $\begin{array}{l}\text { Clam Bay South, off mouth of } \\
\text { Little Clam Bay }\end{array}$ & MVS-056 & $14 \mathrm{~J}$ & $6 \mathrm{~J}$ & 28 & ND & $0.9 \mathrm{U}$ \\
\hline $\begin{array}{l}\text { Port Orchard Passage, Fletcher } \\
\text { Bay entrance }\end{array}$ & MVS-058 & $27 \mathrm{~J}$ & $14 \mathrm{~J}$ & 47 & $2.1 \mathrm{~J}$ & ND \\
\hline \begin{tabular}{|l|} 
Port Orchard Passage, \\
Brownsville \\
\end{tabular} & MVS-059 & 32 & $6 \mathrm{~J}$ & 53 & $1.5 \mathrm{~J}$ & ND \\
\hline OU B Marine (500-ft grid) & OUBM-G 01 & 99 & 52 & 137 & $2.7 \mathrm{~J}$ & $1.4 \mathrm{~J}$ \\
\hline OU B Marine (500-ft grid) & OUBM-G 02 & 94 & 48 & 96 & $1.7 \mathrm{~J}$ & $1.0 \mathrm{~J}$ \\
\hline OU B Marine (500-ft grid) & OUBM-G 03 & 71 & 33 & 106 & $1.5 \mathrm{~J}$ & $1.3 \mathrm{~J}$ \\
\hline OU B Marine (500-ft grid) & OUBM-G 04 & 87 & 45 & 99 & $1.6 \mathrm{~J}$ & $0.7 \mathrm{U}$ \\
\hline OU B Marine (500-ft grid) & OUBM-G 05 & 78 & 49 & 102 & $1.5 \mathrm{~J}$ & $0.9 \mathrm{U}$ \\
\hline OU B Marine (500-ft grid) & OUBM-G 06 & 106 & 59 & 129 & $1.0 \mathrm{~J}$ & $2.4 \mathrm{~J}$ \\
\hline OU B Marine (500-ft grid) & OUBM-G 07 & 78 & 43 & 108 & $1.1 \mathrm{~J}$ & $1.1 \mathrm{~J}$ \\
\hline OU B Marine (500-ft grid) & OUBM-G 08 & 105 & 50 & 115 & $0.2 \mathrm{U}$ & $2.2 \mathrm{~J}$ \\
\hline OU B Marine (500-ft grid) & OUBM-G 09 & 108 & 66 & 138 & $0.0 \mathrm{U}$ & $1.3 \mathrm{~J}$ \\
\hline OU B Marine (500-ft grid) & OUBM-G 10 & 63 & 31 & 89 & $0.8 \mathrm{U}$ & $0.5 \mathrm{U}$ \\
\hline OU B Marine (500-ft grid) & OUBM-G 11 & 65 & 31 & 87 & $1.1 \mathrm{~J}$ & $1.9 \mathrm{~J}$ \\
\hline OU B Marine (500-ft grid) & OUBM-G 12 & 46 & 25 & 76 & $1.6 \mathrm{~J}$ & $0.1 \mathrm{U}$ \\
\hline OU B Marine (500-ft grid) & OUBM-G 13 & 80 & 51 & 110 & $1.6 \mathrm{~J}$ & $1.2 \mathrm{~J}$ \\
\hline OU B Marine (500-ft grid) & OUBM-G 14 & 69 & 43 & 104 & $0.9 \mathrm{U}$ & $0.2 \mathrm{U}$ \\
\hline OU B Marine (500-ft grid) & OUBM-G 15 & 58 & 28 & 88 & $2.4 \mathrm{~J}$ & $0.0 \mathrm{U}$ \\
\hline
\end{tabular}


Table 5. (continued)

\begin{tabular}{|c|c|c|c|c|c|c|}
\hline \multirow{2}{*}{ Location Description } & \multirow{2}{*}{$\begin{array}{c}\text { Station or OUB } \\
\text { Grid Cell }\end{array}$} & \multicolumn{5}{|c|}{ XRF Screening Concentration (mg/kg) } \\
\hline & & $\mathrm{Cu}$ & $\mathbf{P b}$ & Zn & Cd & Ag \\
\hline \multicolumn{2}{|c|}{ SQS } & 390 & 450 & 410 & 5.1 & 6.1 \\
\hline \multicolumn{2}{|c|}{$90 \%$ SQS } & 351 & 405 & 369 & 4.6 & 5.5 \\
\hline \multicolumn{2}{|c|}{ MCUL } & 390 & 530 & 960 & 6.7 & 6.1 \\
\hline OU B Marine (500-ft grid) & OUBM-G 16 & 51 & 28 & 83 & $1.8 \mathrm{~J}$ & $0.9 \mathrm{U}$ \\
\hline OU B Marine (500-ft grid) & OUBM-G 17 & 68 & 36 & 97 & $0.1 \mathrm{U}$ & $1.4 \mathrm{~J}$ \\
\hline OU B Marine (500-ft grid) & OUBM-G 18 & 57 & 31 & 76 & ND & $0.1 \mathrm{U}$ \\
\hline OU B Marine (500-ft grid) & OUBM-G 19 & 62 & 30 & 91 & $1.4 \mathrm{~J}$ & $1.0 \mathrm{~J}$ \\
\hline OU B Marine (500-ft grid) & OUBM-G 20 & 66 & 39 & 92 & $0.7 \mathrm{U}$ & $0.8 \mathrm{U}$ \\
\hline OU B Marine (500-ft grid) & OUBM-G 21 & 78 & 31 & 85 & ND & ND \\
\hline OU B Marine (500-ft grid) & OUBM-G 22 & 79 & 48 & 103 & $1.9 \mathrm{~J}$ & $0.5 \mathrm{U}$ \\
\hline OU B Marine (500-ft grid) & OUBM-G 23 & 92 & 45 & 102 & $1.6 \mathrm{~J}$ & $1.2 \mathrm{~J}$ \\
\hline OU B Marine (500-ft grid) & OUBM-G 24 & 114 & 44 & 131 & $1.5 \mathrm{~J}$ & ND \\
\hline OU B Marine (500-ft grid) & OUBM-G 25 & 134 & 80 & 181 & $2.2 \mathrm{~J}$ & $2.2 \mathrm{~J}$ \\
\hline OU B Marine (500-ft grid) & OUBM-G 26 & 138 & 66 & 147 & $1.4 \mathrm{~J}$ & $1.9 \mathrm{~J}$ \\
\hline OU B Marine (500-ft grid) & OUBM-G 27 & 83 & 41 & 106 & $0.3 \mathrm{U}$ & ND \\
\hline OU B Marine (500-ft grid) & OUBM-G 28 & 122 & 42 & 134 & $0.6 \mathrm{U}$ & $0.6 \mathrm{U}$ \\
\hline OU B Marine (500-ft grid) & OUBM-G 29 & 146 & 102 & 166 & $2.3 \mathrm{~J}$ & $1.1 \mathrm{~J}$ \\
\hline OU B Marine (500-ft grid) & OUBM-G 30 & 108 & 49 & 120 & $2.1 \mathrm{~J}$ & $1.6 \mathrm{~J}$ \\
\hline OU B Marine (500-ft grid) & OUBM-G 31 & 83 & 45 & 101 & $1.0 \mathrm{~J}$ & $0.7 \mathrm{U}$ \\
\hline OU B Marine (500-ft grid) & OUBM-G 32 & 87 & 41 & 105 & $0.6 \mathrm{U}$ & $0.1 \mathrm{U}$ \\
\hline OU B Marine (500-ft grid) & OUBM-G 33 & 135 & 64 & 168 & $1.8 \mathrm{~J}$ & $0.6 \mathrm{U}$ \\
\hline OU B Marine (500-ft grid) & OUBM-G 34 & 152 & 98 & 247 & $2.0 \mathrm{~J}$ & $1.0 \mathrm{~J}$ \\
\hline OU B Marine (500-ft grid) & OUBM-G 35 & 80 & 43 & 93 & $0.3 \mathrm{U}$ & $0.1 \mathrm{U}$ \\
\hline OU B Marine (500-ft grid) & OUBM-G 36 & 105 & 56 & 133 & $0.4 \mathrm{U}$ & $0.6 \mathrm{U}$ \\
\hline OU B Marine (500-ft grid) & OUBM-G 37 & 98 & 55 & 132 & ND & $0.6 \mathrm{U}$ \\
\hline OU B Marine (500-ft grid) & OUBM-G 38 & 97 & 63 & 131 & $0.1 \mathrm{U}$ & $0.6 \mathrm{U}$ \\
\hline OU B Marine (500-ft grid) & OUBM-G 39 & 181 & 94 & $425^{d}$ & $0.7 \mathrm{U}$ & $1.3 \mathrm{~J}$ \\
\hline OU B Marine (500-ft grid) & OUBM-G 40 & 99 & 55 & 142 & ND & ND \\
\hline OU B Marine (500-ft grid) & OUBM-G 41 & 89 & 47 & 125 & $0.8 \mathrm{U}$ & $0.6 \mathrm{U}$ \\
\hline OU B Marine (500-ft grid) & OUBM-G 42 & 100 & 52 & 129 & $0.0 \mathrm{U}$ & $0.1 \mathrm{U}$ \\
\hline OU B Marine (500-ft grid) & OUBM-G 43 & 106 & 49 & 148 & $2.6 \mathrm{~J}$ & ND \\
\hline OU B Marine (500-ft grid) & OUBM-G 44 & 85 & 46 & 113 & ND & $0.9 \mathrm{U}$ \\
\hline OU B Marine (500-ft grid) & OUBM-G 45 & 114 & 60 & 151 & $2.2 \mathrm{~J}$ & $1.5 \mathrm{~J}$ \\
\hline OU B Marine (500-ft grid) & OUBM-G 46 & 133 & 92 & 286 & $1.7 \mathrm{~J}$ & $1.8 \mathrm{~J}$ \\
\hline OU B Marine (500-ft grid) & OUBM-G 47 & 84 & 45 & 125 & $0.3 \mathrm{U}$ & $0.4 \mathrm{U}$ \\
\hline OU B Marine (500-ft grid) & OUBM-G 48 & 70 & 34 & 90 & ND & $1.2 \mathrm{~J}$ \\
\hline OU B Marine (500-ft grid) & OUBM-G 49 & 146 & 107 & 250 & $0.3 \mathrm{U}$ & $0.9 \mathrm{U}$ \\
\hline OU B Marine (500-ft grid) & OUBM-G 50 & 92 & 38 & 113 & $0.2 \mathrm{U}$ & $\mathrm{ND}$ \\
\hline
\end{tabular}


Table 5. (continued)

\begin{tabular}{|c|c|c|c|c|c|c|}
\hline \multirow[b]{2}{*}{ Location Description } & \multirow{2}{*}{$\begin{array}{c}\text { Station or OUB } \\
\text { Grid Cell }\end{array}$} & \multicolumn{5}{|c|}{ XRF Screening Concentration (mg/kg) } \\
\hline & & \multirow{4}{*}{$\begin{array}{c}\mathrm{Cu} \\
390 \\
351 \\
390\end{array}$} & \multirow{4}{*}{$\begin{array}{c}\mathrm{Pb} \\
450 \\
405 \\
530 \\
\end{array}$} & \multirow{4}{*}{$\begin{array}{r}\text { Zn } \\
410 \\
369 \\
960\end{array}$} & \multirow{4}{*}{$\begin{array}{l}\text { Cd } \\
5.1 \\
4.6 \\
6.7\end{array}$} & \multirow{4}{*}{$\begin{array}{l}\mathrm{Ag} \\
6.1 \\
5.5 \\
6.1\end{array}$} \\
\hline SQ & & & & & & \\
\hline $90 \% \mathrm{SQ}$ & & & & & & \\
\hline MCUI & & & & & & \\
\hline OU B Marine (500-ft grid) & OUBM-G 51 & 65 & 36 & 95 & ND & $0.4 \mathrm{U}$ \\
\hline OU B Marine (500-ft grid) & OUBM-G 52 & 247 & 171 & 417 & $1.1 \mathrm{~J}$ & $0.8 \mathrm{U}$ \\
\hline OU B Marine (500-ft grid) & OUBM-G 53 & 74 & 45 & 112 & $0.8 \mathrm{U}$ & ND \\
\hline OU B Marine (500-ft grid) & OUBM-G 54 & 68 & 41 & 105 & $1.5 \mathrm{~J}$ & $1.7 \mathrm{~J}$ \\
\hline OU B Marine (500-ft grid) & OUBM-G 55 & 133 & 65 & 188 & $2.2 \mathrm{~J}$ & ND \\
\hline OU B Marine (500-ft grid) & OUBM-G 56 & 122 & 61 & 166 & $1.5 \mathrm{~J}$ & $0.5 \mathrm{U}$ \\
\hline OU B Marine (500-ft grid) & OUBM-G 57 & 100 & 87 & 177 & $1.2 \mathrm{~J}$ & ND \\
\hline OU B Marine (500-ft grid) & OUBM-G 58 & 71 & 41 & 104 & $1.1 \mathrm{~J}$ & $0.3 \mathrm{U}$ \\
\hline OU B Marine (500-ft grid) & OUBM-G 59 & 152 & 117 & 280 & $1.4 \mathrm{~J}$ & $2.4 \mathrm{~J}$ \\
\hline OU B Marine (500-ft grid) & OUBM-G 60 & 126 & 75 & 291 & $1.1 \mathrm{~J}$ & $1.2 \mathrm{~J}$ \\
\hline OU B Marine (500-ft grid) & OUBM-G 61 & 75 & 167 & 191 & $0.1 \mathrm{U}$ & $0.1 \mathrm{U}$ \\
\hline OU B Marine (500-ft grid) & OUBM-G 62 & 67 & 48 & 109 & $1.4 \mathrm{~J}$ & $0.2 \mathrm{U}$ \\
\hline OU B Marine (500-ft grid) & OUBM-G 63 & 192 & 107 & 253 & $0.0 \mathrm{U}$ & $0.0 \mathrm{U}$ \\
\hline OU B Marine (500-ft grid) & OUBM-G 64 & 149 & 113 & 279 & $1.5 \mathrm{~J}$ & ND \\
\hline OU B Marine (500-ft grid) & OUBM-G 65 & 118 & 70 & 197 & ND & $1.1 \mathrm{~J}$ \\
\hline OU B Marine (500-ft grid) & OUBM-G 66 & 87 & 66 & 249 & $2.0 \mathrm{~J}$ & $0.7 \mathrm{U}$ \\
\hline OU B Marine (500-ft grid) & OUBM-G 67 & 211 & 140 & 283 & $1.9 \mathrm{~J}$ & $0.4 \mathrm{U}$ \\
\hline OU B Marine (500-ft grid) & OUBM-G 68 & 129 & 67 & 558 & $1.2 \mathrm{~J}$ & $1.4 \mathrm{~J}$ \\
\hline OU B Marine (500-ft grid) & OUBM-G 69 & 79 & 50 & 117 & $1.2 \mathrm{~J}$ & $0.1 \mathrm{U}$ \\
\hline OU B Marine (500-ft grid) & OUBM-G 70 & 69 & 43 & 103 & $0.3 \mathrm{U}$ & $1.9 \mathrm{~J}$ \\
\hline OU B Marine (500-ft grid) & OUBM-G 71 & 49 & 27 & 61 & ND & ND \\
\hline Outside OU B (1500-ft grid) & OOUB-G 01 & 63 & 102 & 260 & $0.5 \mathrm{U}$ & $0.9 \mathrm{U}$ \\
\hline Outside OU B (1500-ft grid) & OOUB-G 02 & $23 \mathrm{~J}$ & $13 \mathrm{~J}$ & 42 & $0.1 \mathrm{U}$ & $0.6 \mathrm{U}$ \\
\hline Outside OU B (1500-ft grid) & OOUB-G 03 & 36 & $13 \mathrm{~J}$ & 53 & $0.1 \mathrm{U}$ & $1.0 \mathrm{~J}$ \\
\hline Outside OU B (1500-ft grid) & OOUB-G 04 & $21 \mathrm{~J}$ & $12 \mathrm{~J}$ & 52 & $0.2 \mathrm{U}$ & $0.6 \mathrm{U}$ \\
\hline Outside OU B (1500-ft grid) & OOUB-G 05 & 74 & 31 & 91 & $1.6 \mathrm{~J}$ & $1.2 \mathrm{~J}$ \\
\hline Outside OU B (1500-ft grid) & OOUB-G 06 & 78 & 39 & 100 & $1.4 \mathrm{~J}$ & $1.3 \mathrm{~J}$ \\
\hline Outside OU B (1500-ft grid) & OOUB-G 07 & 92 & 45 & 110 & $1.9 \mathrm{~J}$ & $1.7 \mathrm{~J}$ \\
\hline Outside OU B (1500-ft grid) & OOUB-G 08 & 100 & 46 & 102 & $1.1 \mathrm{~J}$ & $0.7 \mathrm{U}$ \\
\hline Outside OU B (1500-ft grid) & OOUB-G 09 & 86 & 41 & 109 & $0.9 \mathrm{U}$ & $0.3 \mathrm{U}$ \\
\hline \begin{tabular}{|l|} 
Outside OU B (1500-ft grid) \\
\end{tabular} & OOUB-G 10 & 79 & 44 & 101 & $0.5 \mathrm{U}$ & $0.1 \mathrm{U}$ \\
\hline Outside OU B (1500-ft grid) & OOUB-G 11 & 85 & 44 & 94 & $0.5 \mathrm{U}$ & $0.2 \mathrm{U}$ \\
\hline Outside OU B (1500-ft grid) & OOUB-G 12 & 33 & 16 & 51 & ND & $0.3 \mathrm{U}$ \\
\hline Outside OU B (1500-ft grid) & OOUB-G 13 & 69 & 49 & 114 & ND & $0.2 \mathrm{U}$ \\
\hline Outside OU B (1500-ft grid) & OOUB-G 14 & 73 & 40 & 116 & ND & $0.7 \mathrm{U}$ \\
\hline Outside OU B (1500-ft grid) & OOUB-G 15 & 79 & 39 & 96 & $2.6 \mathrm{~J}$ & $1.0 \mathrm{~J}$ \\
\hline
\end{tabular}


Table 5. (continued)

\begin{tabular}{|c|c|c|c|c|c|c|}
\hline \multirow[b]{2}{*}{ Location Description } & \multirow{2}{*}{$\begin{array}{c}\text { Station or OUB } \\
\text { Grid Cell }\end{array}$} & \multicolumn{5}{|c|}{ XRF Screening Concentration (mg/kg) } \\
\hline & & \multirow{2}{*}{$\begin{array}{c}\mathrm{Cu} \\
390\end{array}$} & \multirow{2}{*}{$\begin{array}{c}\mathrm{Pb} \\
450\end{array}$} & \multirow{2}{*}{$\begin{array}{r}\mathrm{Zn} \\
410\end{array}$} & \multirow{2}{*}{$\begin{array}{l}\text { Cd } \\
5.1\end{array}$} & \multirow{2}{*}{$\begin{array}{l}\mathrm{Ag} \\
6.1\end{array}$} \\
\hline SQS & & & & & & \\
\hline $90 \%$ SQS & & 351 & 405 & 369 & 4.6 & 5.5 \\
\hline \multicolumn{2}{|l|}{ MCUL } & 390 & 530 & 960 & 6.7 & 6.1 \\
\hline Outside OU B (1500-ft grid) & OOUB-G 16 & 76 & 39 & 114 & $1.3 \mathrm{~J}$ & ND \\
\hline Outside OU B (1500-ft grid) & OOUB-G 17 & 75 & 46 & 97 & $1.7 \mathrm{~J}$ & $0.8 \mathrm{U}$ \\
\hline Outside OU B (1500-ft grid) & OOUB-G 18 & 88 & 44 & 100 & ND & $0.1 \mathrm{U}$ \\
\hline Outside OU B (1500-ft grid) & OOUB-G 19 & 69 & 43 & 101 & $1.2 \mathrm{~J}$ & $1.7 \mathrm{~J}$ \\
\hline Outside OU B (1500-ft grid) & OOUB-G 20 & 63 & 34 & 84 & $0.2 \mathrm{U}$ & $2.6 \mathrm{~J}$ \\
\hline Outside OU B (1500-ft grid) & OOUB-G 21 & 75 & 47 & 102 & $1.4 \mathrm{~J}$ & $0.9 \mathrm{U}$ \\
\hline Outside OU B (1500-ft grid) & OOUB-G 22 & 65 & 43 & 92 & $1.8 \mathrm{~J}$ & $0.8 \mathrm{U}$ \\
\hline Outside OU B (1500-ft grid) & OOUB-G 23 & 62 & 31 & 79 & $0.3 \mathrm{U}$ & ND \\
\hline Outside OU B (1500-ft grid) & OOUB-G 24 & 84 & 49 & 102 & $0.3 \mathrm{U}$ & $1.2 \mathrm{~J}$ \\
\hline Outside OU B (1500-ft grid) & OOUB-G 25 & 60 & 32 & 87 & $0.3 \mathrm{U}$ & ND \\
\hline Outside OU B (1500-ft grid) & OOUB-G 26 & $16 \mathrm{~J}$ & $9 \mathrm{~J}$ & 40 & $1.1 \mathrm{~J}$ & ND \\
\hline Outside OU B (1500-ft grid) & OOUB-G 27 & 58 & 34 & 87 & $2.4 \mathrm{~J}$ & $0.7 \mathrm{U}$ \\
\hline Outside OU B (1500-ft grid) & OOUB-G 28 & 66 & 38 & 99 & $0.3 \mathrm{U}$ & $1.5 \mathrm{~J}$ \\
\hline Outside OU B (1500-ft grid) & OOUB-G 29 & 43 & 28 & 79 & $0.4 \mathrm{U}$ & $0.5 \mathrm{U}$ \\
\hline Outside OU B (1500-ft grid) & OOUB-G 30 & $15 \mathrm{~J}$ & $11 \mathrm{~J}$ & 42 & ND & $\mathrm{ND}$ \\
\hline Outside OU B (1500-ft grid) & OOUB-G 31 & 62 & 39 & 96 & $0.4 \mathrm{U}$ & $0.9 \mathrm{U}$ \\
\hline Outside OU B (1500-ft grid) & OOUB-G 32 & 45 & 31 & 76 & ND & $0.8 \mathrm{U}$ \\
\hline
\end{tabular}
a. J Estimated value above method detection limit but below quantitation limit.
b. U Value is less than method detection limit.
c. ND The analyte was not detected.
d. Bold text in highlighted box indicates XRF result $>90 \%$ SQS.

XRF data were examined to identify samples that met the selection criteria for confirmatory analysis.

The criterion for concentrations $\geq 90 \%$ SQS was applied two ways: first, directly to the XRF results, and second, to predicted concentrations calculated using the existing linear correlation for Sinclair-Dyes Inlets that was generated during the Mass Balance study (Figure 11, Miller et al. 2003). The 40 samples (25\%) selected for confirmatory analysis by ICP-MS and the rationale for their selection are provided in Table 6; the number of samples from each geographical area of the Sinclair-Dyes Inlet study area is summarized in Table 7. 
Table 6. Samples Selected for Confirmatory Metals Analysis by ICP-MS

\begin{tabular}{|c|c|c|}
\hline Selection Criteria & Selected Samples & Comments \\
\hline XRF result $\geq 90 \%$ SQS & OUBM-G39, G52, G68 & \\
\hline $\begin{array}{l}\text { Predicted concentration } \geq \\
90 \% \text { SQS (calculated by } \\
\text { applying Mass Balance } \\
\text { XRF:ICP-MS correlation } \\
\text { to present XRF result ) }\end{array}$ & OUBM-G46, G59, G60, G64, G67 & Most are located within listed segments \\
\hline $\begin{array}{l}\text { At least } 3 \text { samples per } \\
\text { 303(d) segment listed for } \\
\text { Cd or Ag }\end{array}$ & $\begin{array}{l}\text { OUBM- G34, G36, G40, G42, } \\
\text { G43, G45, G56 } \\
\text { OOUB-G27 } \\
\text { MVS-019, 024, 025, 026, 027, } 022\end{array}$ & $\begin{array}{l}\text { Additional samples in listed segments, } \\
\text { also covers high end of concentration } \\
\text { range and possible spatial gradient from } \\
\text { nearshore samples } \\
\text { OOUB-G27 is only 1500-ft grid entirely } \\
\text { within a listed segment } \\
\text { Located in Ostrich Bay }\end{array}$ \\
\hline $\begin{array}{l}\text { XRF result much different } \\
\text { from expected }\end{array}$ & None & No samples met this criterion \\
\hline $\begin{array}{l}\text { Representative of } \\
\text { concentration range, and/or } \\
\text { spatial distribution }\end{array}$ & $\begin{array}{l}\text { OUBM-G66 } \\
\text { OOUB-G21, G24, G28 } \\
\text { OOUB-G01, G07, G13, G18, G26 } \\
\text { MVS-002, 007, 013, 034, 039, } \\
\text { 043, 047, } 056\end{array}$ & $\begin{array}{l}\text { High end of concentration range } \\
\text { Border listed segments; possible gradient } \\
\text { from nearshore stations } \\
\text { Spatial representation } \\
\text { Spatial representation, distribution }\end{array}$ \\
\hline
\end{tabular}

Table 7. Metals Verification Study Sample Analysis Summary

\begin{tabular}{||c|c|c||}
\hline \multicolumn{1}{|c|}{ Location } & $\begin{array}{c}\text { Number of XRF } \\
\text { Screening Samples }\end{array}$ & $\begin{array}{c}\text { Number of ICP-MS } \\
\text { Confirmatory Samples }\end{array}$ \\
\hline Sinclair Inlet, Inside PSNS OU-B & 71 & 17 \\
\hline Sinclair Inlet, Outside PSNS OU-B & 32 & 26 \\
\hline Sinclair Inlet Subtotal & 103 & 6 \\
\hline Ostrich \& Oyster Bays & & 4 \\
\hline Dyes Inlet (outside Ostrich Bay), Phinney Bay & 12 & 4 \\
\hline Port Orchard Passage and Rich Passage & 19 & 14 \\
\hline $\begin{array}{r}\text { Dyes Inlet, Port Washington Narrows, } \\
\text { Port Orchard \& Rich Passages Subtotal }\end{array}$ & 57 & \\
\hline \hline TOTAL & & 40 \\
\hline \hline
\end{tabular}


The results of confirmatory, quantitative metals analysis by ICP-MS on selected Metals Verification

Study samples are provided in Table 8. The laboratory data report for ICP-MS analysis, including narrative summary and QC data, is provided in Appendix B. The ICP-MS quantitative data were validated by acceptable results for all QC measures. Target metals were undetected in the method blanks, and precision between laboratory duplicates was very good with relative percent differences (RPD) between duplicates of $1 \%$ to $8 \%$ (QC criterion is $\pm 30 \%$ ). Accuracy of measurements was assessed three different ways, with acceptable results for all three: matrix spike recoveries were $77 \%$ to $122 \%$ and laboratory control sample (blank spike) recoveries were $90 \%$ to $115 \%$, both within the QC criterion range of $70 \%$ to $130 \%$; standard reference material results were within $20 \%$ of the certified values.

\subsection{Correlation of Screening and Quantitative Results}

To predict actual metal concentrations from XRF screening data, quantitative analytical results for the 40 confirmatory samples were plotted against screening results for the same samples. Positive linear correlations were developed for $\mathrm{Pb}, \mathrm{Cu}$, and $\mathrm{Zn}$, which were the only metals reliably detected by XRF in the majority of samples (Figure 16). There was one outlier sample for each metal, probably due to sample inhomogeneity as the outlier samples were all located in between PSNS piers where variable particulate metal concentrations are expected (OUBM-G59, -G60, and -G67, Figure 14). When these outlier values were removed from the correlation, the XRF results for $\mathrm{Cu}$ and $\mathrm{Pb}$ correlated very well with ICP-MS results ( $\mathrm{R}^{2}$ values were 0.876 for $\mathrm{Cu}, 0.932$ for $\mathrm{Pb}$ ). Zn measured by XRF correlated well with ICP-MS results ( $\mathrm{R}^{2}$ of 0.730 ), especially up to XRF concentrations of $200 \mathrm{ppm}$. However, when the XRF concentration was $250 \mathrm{ppm} \mathrm{Zn}$ or higher, there was much more variability between data points and the linear relationship between the two methods did not appear to be as strong. The resulting linear equations with outliers removed were used to predict a definitive metal concentration from the XRF result for those samples that did not receive confirmatory analysis (Table 9). 
Table 8. Confirmatory Metals Analysis Results, Metals Verification Study

\begin{tabular}{|c|c|c|c|c|c|c|c|c|c|}
\hline \multirow[b]{2}{*}{ Location Description } & \multirow{2}{*}{$\begin{array}{l}\text { MVS Station or } \\
\text { OU B Grid Cell }\end{array}$} & \multicolumn{8}{|c|}{ Metal Concentration (mg/kg dry wt) } \\
\hline & & $\mathbf{A g}^{\mathbf{a}}$ & $\mathrm{Cr}$ & $\mathbf{N i}$ & Cu & Zn & As & Cd & $\mathbf{P b}$ \\
\hline Dyes Depositional Zone 1 (North) & MVS-002 & 0.120 & 60.0 & 22.8 & 16.4 & 46.6 & 4.17 & 0.783 & 12.2 \\
\hline Dyes Depositional Zone 2 (West) & MVS-007 & 0.790 & 96.8 & 49.0 & 78.8 & 159 & 14.8 & 1.26 & 63.8 \\
\hline Dyes Depositional Zone 3 (SW) & MVS-013 & 0.609 & 98.7 & 45.6 & 63.0 & 140 & 12.3 & 1.07 & 52.9 \\
\hline Dyes Depositional Zone 4 (South) & MVS-019 & 0.156 & 75.7 & 31.6 & 25.7 & 66.1 & 6.38 & 0.631 & 17.6 \\
\hline Dyes Depositional Zone 4 (South) & MVS-022 & 0.681 & 113 & 45.9 & 65.6 & 132 & 11.8 & 1.80 & 45.4 \\
\hline Ostrich Bay & MVS-024 & 0.484 & 95.9 & 41.2 & 54.6 & 113 & 10.4 & 0.986 & 41.7 \\
\hline Ostrich Bay & MVS-025 & 0.544 & 91.1 & 43.1 & 58.7 & 118 & 11.2 & 1.17 & 43.6 \\
\hline Ostrich Bay & MVS-026 & 0.489 & 100 & 41.6 & 52.7 & 112 & 10.6 & 1.15 & 39.4 \\
\hline Ostrich Bay & MVS-027 & 0.611 & 102 & 44.2 & 62.3 & 126 & 10.9 & 1.53 & 44.0 \\
\hline Ostrich Bay & MVS-028 & 0.127 & 67.4 & 30.5 & 17.6 & 50.9 & 5.60 & 0.525 & 12.2 \\
\hline Dyes Inlet Nondepositional (East) & MVS-034 & 0.063 & 71.7 & 24.3 & 10.4 & 41.1 & 3.64 & 0.206 & 9.50 \\
\hline Port Orchard Passage & MVS-039 & 0.056 & 43.4 & 27.3 & 11.8 & 47.9 & 4.04 & 0.136 & 11.1 \\
\hline Port Orchard Passage & MVS-043 & 0.056 & 52.1 & 19.1 & 9.56 & 37.9 & 3.83 & 0.184 & 11.1 \\
\hline Port Orchard Passage & MVS-047 & 0.534 & 81.3 & 43.7 & 58.8 & 126 & 13.4 & 0.846 & 41.6 \\
\hline $\begin{array}{l}\text { Clam Bay South, off mouth of Little } \\
\text { Clam Bay }\end{array}$ & MVS-056 & 0.080 & 46.7 & 15.7 & 11.8 & 43.4 & 4.29 & 0.656 & 8.69 \\
\hline OU-B Marine (500-ft grid) & OUBM-G 34 & 2.38 & 91.8 & 45.4 & 171 & 391 & 20.2 & 1.51 & 132 \\
\hline OU-B Marine (500-ft grid) & OUBM-G 36 & 0.838 & 99.6 & 59.5 & 150 & 185 & 14.9 & 1.09 & 86.3 \\
\hline OU-B Marine (500-ft grid) & OUBM-G 39 & 1.11 & 84.1 & 40.6 & 173 & 288 & 11.9 & 1.39 & 128 \\
\hline OU-B Marine (500-ft grid) & OUBM-G 40 & 0.709 & 108 & 49.9 & 135 & 208 & 12.1 & 1.33 & 84.9 \\
\hline OU-B Marine (500-ft grid) & OUBM-G 42 & 0.688 & 103 & 77.2 & 180 & 188 & 13.1 & 1.06 & 77.8 \\
\hline OU-B Marine (500-ft grid) & OUBM-G 43 & 0.588 & 84.0 & 51.4 & 155 & 241 & 15.8 & 1.12 & 74.3 \\
\hline OU-B Marine (500-ft grid) & OUBM-G 45 & 0.740 & 88.7 & 48.4 & 154 & 238 & 13.1 & 1.44 & 90.0 \\
\hline OU-B Marine (500-ft grid) & OUBM-G 46 & 0.495 & 105 & 40.1 & 142 & $428 \quad \mathrm{D}^{\mathrm{b}}$ & 15.8 & 0.621 & 155 \\
\hline OU-B Marine (500-ft grid) & OUBM-G 52 & 1.07 & 119 & 79.1 & $398 \mathrm{D}$ & 785 & 36.1 & 1.30 & 279 \\
\hline OU-B Marine (500-ft grid) & OUBM-G 56 & 0.691 & 99.9 & 47.5 & 194 & 324 & 19.6 & 1.54 & 105 \\
\hline OU-B Marine (500-ft grid) & OUBM-G 59 & 0.712 & 102 & 52.9 & 272 & 736 & 23.1 & 3.17 & 439 \\
\hline OU-B Marine (500-ft grid) & OUBM-G 60 & 0.642 & 85.7 & 45.5 & 200 & 1480 & 19.7 & 2.01 & 180 \\
\hline OU-B Marine (500-ft grid) & OUBM-G 64 & 0.777 & 98.1 & 51.7 & 230 & 425 & 19.9 & 1.85 & 209 \\
\hline
\end{tabular}


Table 8. (continued)

\begin{tabular}{|c|c|c|c|c|c|c|c|c|c|}
\hline \multirow[b]{2}{*}{ Location Description } & \multirow{2}{*}{$\begin{array}{l}\text { MVS Station or } \\
\text { OU B Grid Cell }\end{array}$} & \multicolumn{8}{|c|}{ Metal Concentration (mg/kg dry wt) } \\
\hline & & $\mathbf{A g}^{\mathrm{a}}$ & $\mathrm{Cr}$ & $\mathrm{Ni}$ & $\mathrm{Cu}$ & Zn & As & Cd & $\mathbf{P b}$ \\
\hline OU-B Marine (500-ft grid) & OUBM-G 66 & 0.547 & 101 & 46.1 & 227 & $428 \mathrm{D}$ & 15.2 & 1.71 & 159 \\
\hline OU-B Marine (500-ft grid) & OUBM-G 67 & 1.03 & 104 & 479 & $710 \mathrm{D}$ & $547 \quad \mathrm{D}$ & 43.0 & 1.53 & 204 \\
\hline OU-B Marine (500-ft grid) & OUBM-G 68 & 0.762 & 103 & 54.7 & 217 & $526 \mathrm{D}$ & 20.6 & 1.72 & 144 \\
\hline Outside OU B Marine (1500-ft grid) & OOUB-G 01 & 0.175 & 114 & 97.0 & 102 & $547 \mathrm{D}$ & 15.3 & 0.479 & 198 \\
\hline Outside OU B Marine (1500-ft grid) & OOUB-G 07 & 1.35 & 109 & 53.4 & 130 & 177 & 17.2 & 2.47 & 72.3 \\
\hline Outside OU B Marine (1500-ft grid) & OOUB-G 13 & 0.971 & 98.0 & 48.0 & 116 & 164 & 14.6 & 1.30 & 83.8 \\
\hline Outside OU B Marine (1500-ft grid) & OOUB-G 18 & 0.943 & 96.4 & 47.7 & 117 & 159 & 15.9 & 1.04 & 75.1 \\
\hline Outside OU B Marine (1500-ft grid) & OOUB-G 21 & 0.715 & 99.7 & 48.6 & 102 & 159 & 15.9 & 1.32 & 71.8 \\
\hline Outside OU B Marine (1500-ft grid) & OOUB-G 24 & 0.705 & 87.4 & 45.4 & 107 & 162 & 13.8 & 0.797 & 72.0 \\
\hline Outside OU B Marine (1500-ft grid) & OOUB-G 26 & 0.042 & 83.6 & 27.0 & 11.7 & 45.4 & 2.67 & 0.193 & 8.53 \\
\hline Outside OU B Marine (1500-ft grid) & OOUB-G 27 & 0.417 & 84.8 & 42.5 & 72.5 & 128 & 11.7 & 1.04 & 55.4 \\
\hline Outside OU B Marine (1500-ft grid) & OOUB-G 28 & 0.500 & 84.5 & 42.6 & 76.1 & 141 & 12.3 & 0.778 & 58.4 \\
\hline
\end{tabular}

a. Ag was analyzed by graphite-furnace atomic absorption for comparability with other ENVVEST data; all other metals analyzed by ICP-MS.

b. D Diluted analysis (100x) 

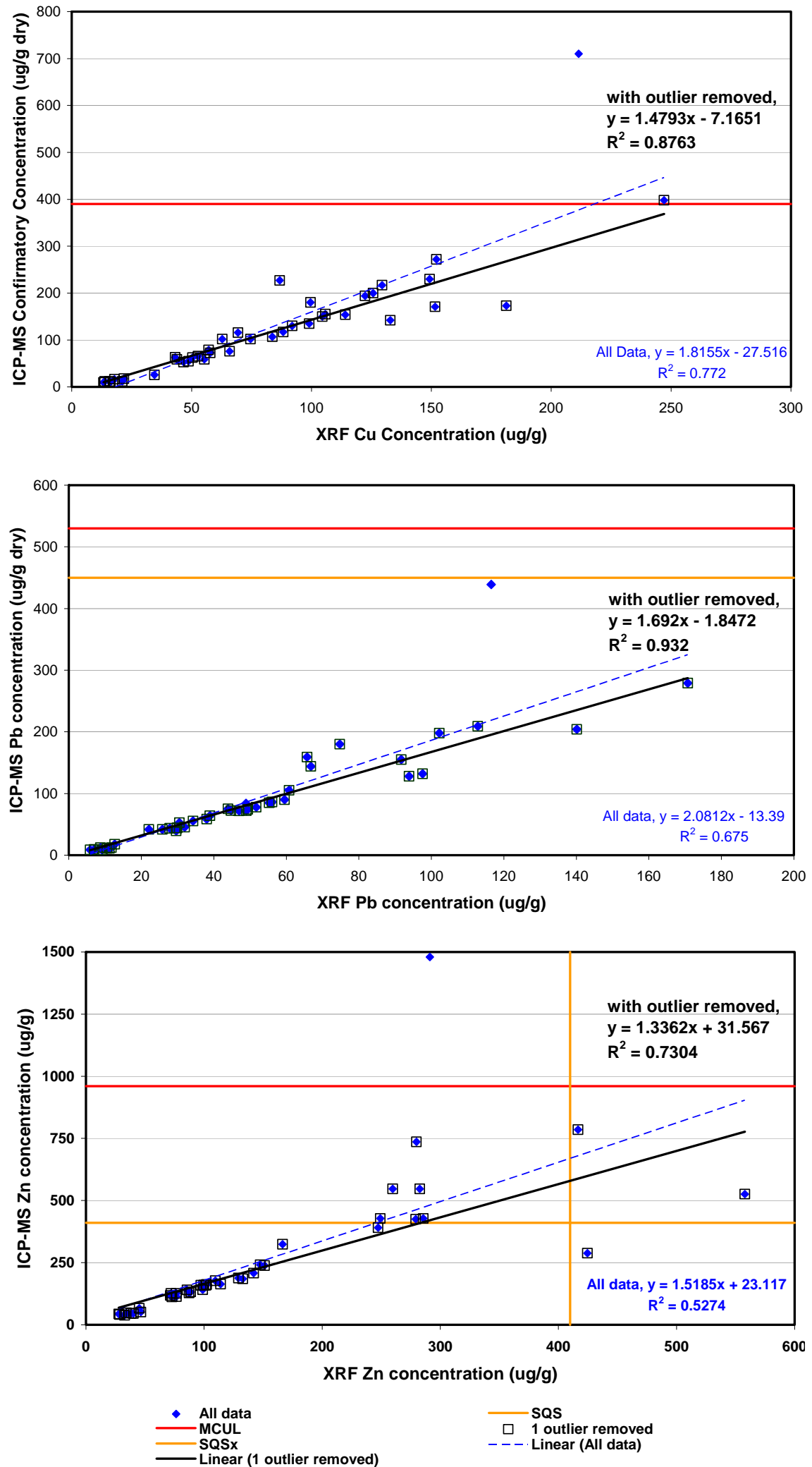

Figure 16. Linear correlations of XRF and ICP-MS measurements for Cu (top), Pb (middle), Zn (bottom), ENVVEST Metals Verification Study 
Table 9. Definitive Sediment Metals Concentrations, Metals Verification Study

\begin{tabular}{|c|c|c|c|c|c|c|c|c|c|}
\hline \multirow{4}{*}{$\begin{array}{l}\text { Location Description } \\
\qquad \begin{array}{r}\text { SQS } \\
\text { MCUL }\end{array} \\
\end{array}$} & \multirow{4}{*}{$\begin{array}{c}\text { Station or OUB } \\
\text { Grid Cell }\end{array}$} & \multirow{4}{*}{$\begin{array}{l}\text { Confirm } \\
\text {-atory } \\
\text { Sample? }\end{array}$} & \multicolumn{7}{|c|}{ Definitive $^{\mathrm{a}}$ Concentration (mg/kg dry weight) } \\
\hline & & & \multirow{3}{*}{$\begin{array}{l}\mathrm{Cu} \\
390 \\
390 \\
\end{array}$} & \multirow{3}{*}{$\begin{array}{l}\mathrm{Pb} \\
450 \\
530 \\
\end{array}$} & \multirow{3}{*}{$\begin{array}{l}\mathrm{Zn} \\
410 \\
960 \\
\end{array}$} & \multicolumn{2}{|l|}{$\mathbf{C d}^{\mathbf{b}}$} & \multicolumn{2}{|c|}{$\mathbf{A g}^{\mathbf{b}}$} \\
\hline & & & & & & 5.1 & & 6.1 & \\
\hline & & & & & & 6.7 & & 6.1 & \\
\hline Dyes Depositional Zone & MVS-001 & No & 44 & 27.5 & 109 & 1.2 & $\mathrm{~J}^{\mathrm{c}}$ & 0.1 & $\mathrm{U}^{\mathrm{d}}$ \\
\hline Dyes Depositional Zone & MVS-002 & Yes & 16 & 12.2 & 47 & 0.783 & & 0.12 & \\
\hline Dyes Depositional Zone & MVS-003 & No & 61 & 48.5 & 144 & 0.5 & $\mathrm{U}$ & 0.3 & $\mathrm{U}$ \\
\hline Dyes Depositional Zone & MVS-004 & No & 86 & 68.0 & 163 & ND & & 0.9 & $\mathrm{U}$ \\
\hline Dyes Depositional Zone & MVS-005 & No & 76 & 62.6 & 153 & 0.6 & $\mathrm{U}$ & 1.4 & $\mathrm{~J}$ \\
\hline Dyes Depositional Zone & MVS-006 & No & 29 & 25.6 & 101 & 0.8 & $\mathrm{U}$ & 0.4 & $\mathrm{U}$ \\
\hline Dyes Depositional Zone & MVS-007 & Yes & 79 & 63.8 & 159 & 1.26 & & 0.79 & \\
\hline Dyes Depositional Zone & MVS-008 & No & 54 & 38.7 & 145 & ND & & 0.9 & $\mathrm{U}$ \\
\hline Dyes Depositional Zone & MVS-009 & No & 63 & 59.3 & 146 & 0.7 & $\mathrm{U}$ & 1.8 & $\mathrm{~J}$ \\
\hline Dyes Depositional Zone & MVS-010 & No & 63 & 50.3 & 153 & 0.5 & $\mathrm{U}$ & 0.2 & $\mathrm{U}$ \\
\hline Dyes Depositional Zone & MVS-011 & No & 56 & 60.9 & 158 & 0.5 & $\mathrm{U}$ & 0.8 & $\mathrm{U}$ \\
\hline Dyes Depositional Zone & MVS-012 & No & 77 & 63.2 & 157 & 0.4 & $\mathrm{U}$ & 0.6 & $\mathrm{U}$ \\
\hline Dyes Depositional Zone & MVS-013 & Yes & 63 & 52.9 & 140 & 1.07 & & 0.609 & \\
\hline Dyes Depositional Zone & MVS-014 & No & 48 & 23.4 & 114 & ND & & ND & \\
\hline Dyes Depositional Zone & MVS-015 & No & 34 & 13.5 & 110 & 0.0 & $\mathrm{U}$ & 0.4 & $\mathrm{U}$ \\
\hline Dyes Depositional Zone & MVS-016 & No & 43 & 21.5 & 118 & 0.7 & $\mathrm{U}$ & 0.1 & $\mathrm{U}$ \\
\hline Dyes Depositional Zone & MVS-017 & No & 60 & 44.4 & 137 & 0.3 & $\mathrm{U}$ & 0.3 & $\mathrm{U}$ \\
\hline Dyes Depositional Zone & MVS-018 & No & 57 & 59.4 & 144 & 1.5 & $\mathrm{~J}$ & 0.9 & $\mathrm{U}$ \\
\hline Dyes Depositional Zone & MVS-019 & Yes & 26 & 17.6 & 66 & 0.631 & & 0.156 & \\
\hline Dyes Depositional Zone & MVS-020 & No & 59 & 52.5 & 134 & ND & & ND & \\
\hline Dyes Depositional Zone & MVS-021 & No & 60 & 42.7 & 128 & 1.0 & $\mathrm{~J}$ & 1.3 & $\mathrm{~J}$ \\
\hline Dyes Depositional Zone & MVS-022 & Yes & 66 & 45.4 & 132 & 1.80 & & 0.681 & \\
\hline Dyes Inlet Nondepositional & MVS-030 & No & 18 & 11.0 & 73 & 0.8 & $\mathrm{U}$ & ND & \\
\hline Dyes Inlet Nondepositional & MVS-031 & No & 36 & 21.7 & 97 & 1.3 & $\mathrm{~J}$ & ND & \\
\hline Dyes Inlet Nondepositional & MVS-032 & No & 17 & 11.2 & 76 & ND & & ND & \\
\hline Dyes Inlet Nondepositional & MVS-033 & No & 35 & 35.1 & 103 & ND & & 1.3 & $\mathrm{~J}$ \\
\hline Dyes Inlet Nondepositional & MVS-034 & Yes & 10 & 9.5 & 41 & 0.21 & & 0.06 & \\
\hline Dyes Inlet Nondepositional & MVS-035 & No & 17 & 9.7 & 81 & 0.8 & $\mathrm{U}$ & ND & \\
\hline Dyes Inlet Nondepositional & MVS-036 & No & 19 & 14.7 & 76 & ND & & $\mathrm{ND}$ & \\
\hline Ostrich Bay & MVS-023 & No & 75 & 58.5 & 156 & 0.2 & $\mathrm{U}$ & 0.5 & $\mathrm{U}$ \\
\hline Ostrich Bay & MVS-024 & Yes & 55 & 41.7 & 113 & 0.986 & & 0.484 & \\
\hline Ostrich Bay & MVS-025 & Yes & 59 & 43.6 & 118 & 1.17 & & 0.544 & \\
\hline Ostrich Bay & MVS-026 & Yes & 53 & 39.4 & 112 & 1.15 & & 0.489 & \\
\hline Ostrich Bay & MVS-027 & Yes & 62 & 44.0 & 126 & 1.53 & & 0.611 & \\
\hline Ostrich Bay & MVS-028 & Yes & 18 & 12.2 & 51 & 0.525 & & 0.127 & \\
\hline Ostrich Bay & MVS-029 & No & 63 & 50.7 & 153 & 2.2 & $\mathrm{~J}$ & ND & \\
\hline Oyster Bay & MVS-052 & No & 47 & 45.5 & 136 & 2.3 & $\mathrm{~J}$ & 0.4 & $\mathrm{U}$ \\
\hline Phinney Bay & MVS-054 & No & 25 & 18.2 & 93 & 1.3 & $\mathrm{~J}$ & 1.8 & $\mathrm{~J}$ \\
\hline
\end{tabular}


Table 9. (continued)

\begin{tabular}{|c|c|c|c|c|c|c|c|c|c|}
\hline \multirow{4}{*}{$\begin{array}{r}\text { Location Description } \\
\text { SQS } \\
\text { MCUL }\end{array}$} & \multirow{2}{*}{$\begin{array}{c}\text { Station or OUB } \\
\text { Grid Cell }\end{array}$} & \multirow{2}{*}{$\begin{array}{c}\text { Confirm } \\
\text {-atory } \\
\text { Sample? }\end{array}$} & \multicolumn{7}{|c|}{ Definitive $^{\text {a }}$ Concentration (mg/kg dry weight) } \\
\hline & & & $\mathrm{Cu}$ & $\mathbf{P b}$ & Zn & $\mathbf{C d}^{\mathbf{b}}$ & & $\mathbf{A g}^{\mathbf{b}}$ & \\
\hline & & & 390 & 450 & 410 & 5.1 & & 6.1 & \\
\hline & & & 390 & 530 & 960 & 6.7 & & 6.1 & \\
\hline Port Orchard Passage & MVS-037 & No & 24 & 10.4 & 90 & 1.6 & $\mathrm{~J}$ & ND & \\
\hline Port Orchard Passage & MVS-038 & No & 25 & 7.7 & 82 & 0.6 & $\mathrm{U}$ & ND & \\
\hline Port Orchard Passage & MVS-039 & Yes & 12 & 11.1 & 48 & 0.136 & & 0.056 & \\
\hline Port Orchard Passage & MVS-040 & No & 46 & 28.9 & 125 & 0.7 & $\mathrm{U}$ & ND & \\
\hline Port Orchard Passage & MVS-041 & No & 59 & 33.1 & 123 & ND & & ND & \\
\hline Port Orchard Passage & MVS-042 & No & 31 & 28.7 & 101 & 0.7 & $\mathrm{U}$ & 0.3 & $\mathrm{U}$ \\
\hline Port Orchard Passage & MVS-043 & Yes & 10 & 11.1 & 38 & 0.184 & & 0.056 & \\
\hline Port Orchard Passage & MVS-044 & No & 20 & 13.1 & 71 & ND & & 0.0 & $\mathrm{U}$ \\
\hline Port Orchard Passage & MVS-045 & No & 44 & 36.3 & 114 & 0.5 & $\mathrm{U}$ & 0.2 & $\mathrm{U}$ \\
\hline Port Orchard Passage & MVS-046 & No & 37 & 28.7 & 91 & ND & & ND & \\
\hline Port Orchard Passage & MVS-047 & Yes & 59 & 41.6 & 126 & 0.846 & & 0.534 & \\
\hline Port Orchard Passage & MVS-048 & No & 29 & 21.7 & 89 & 0.5 & $\mathrm{U}$ & 1.7 & $\mathrm{~J}$ \\
\hline Port Orchard Passage & MVS-049 & No & 50 & 31.0 & 127 & 1.1 & $\mathrm{~J}$ & 1.2 & $\mathrm{~J}$ \\
\hline $\begin{array}{l}\text { Port Orchard Passage, } \\
\text { Fletcher Bay entrance }\end{array}$ & MVS-058 & No & 33 & 22.2 & 94 & 2.1 & $\mathrm{~J}$ & ND & \\
\hline $\begin{array}{l}\text { Port Orchard Passage, } \\
\text { Brownsville }\end{array}$ & MVS-059 & No & 40 & 9.1 & 102 & 1.5 & $\mathrm{~J}$ & ND & \\
\hline Rich Passage, north & MVS-050 & No & 6 & 12.8 & 63 & 0.4 & $\mathrm{U}$ & 0.5 & $\mathrm{U}$ \\
\hline $\begin{array}{l}\text { Clam Bay north, near } \\
\text { Manchester Pier }\end{array}$ & MVS-055 & No & 12 & 5.5 & 65 & 1.6 & $\mathrm{~J}$ & 0.4 & $\mathrm{U}$ \\
\hline $\begin{array}{l}\text { Clam Bay South, off mouth } \\
\text { of Little Clam Bay }\end{array}$ & MVS-056 & Yes & 12 & 8.7 & 43 & 0.656 & & 0.08 & \\
\hline $\begin{array}{l}\text { Rich Passage, NW of } \\
\text { Orchard Rocks }\end{array}$ & MVS-057 & No & 24 & 14.3 & 82 & 0.3 & $\mathrm{U}$ & 0.7 & $\mathrm{U}$ \\
\hline OU-B Marine (500-ft grid) & OUBM-G & No & 140 & 85.8 & 215 & 2.7 & $\mathrm{~J}$ & 1.4 & $\mathrm{~J}$ \\
\hline OU-B Marine (500-ft grid) & OUBM-G 02 & No & 131 & 80.0 & 160 & 1.7 & $\mathrm{~J}$ & 1.0 & $\mathrm{~J}$ \\
\hline OU-B Marine (500-ft grid) & OUBM-G 03 & No & 98 & 53.8 & 173 & 1.5 & $\mathrm{~J}$ & 1.3 & $\mathrm{~J}$ \\
\hline OU-B Marine (500-ft grid) & OUBM-G 04 & No & 122 & 74.5 & 164 & 1.6 & $\mathrm{~J}$ & 0.7 & $\mathrm{U}$ \\
\hline OU-B Marine (500-ft grid) & OUBM-G 05 & No & 109 & 80.6 & 168 & 1.5 & $\mathrm{~J}$ & 0.9 & $\mathrm{U}$ \\
\hline OU-B Marine (500-ft grid) & OUBM-G 06 & No & 150 & 98.3 & 203 & 1.0 & $\mathrm{~J}$ & 2.4 & $\mathrm{~J}$ \\
\hline OU-B Marine (500-ft grid) & OUBM-G & No & 109 & 71.6 & 175 & 1.1 & $\mathrm{~J}$ & 1.1 & $\mathrm{~J}$ \\
\hline OU-B Marine (500-ft grid) & OUBM-G & No & 149 & 82.3 & 186 & 0.2 & $\mathrm{U}$ & 2.2 & $\mathrm{~J}$ \\
\hline OU-B Marine (500-ft grid) & OUBM-G & No & 152 & 109.3 & 217 & 0.0 & $\mathrm{U}$ & 1.3 & $\mathrm{~J}$ \\
\hline OU-B Marine (500-ft grid) & OUBM-G 10 & No & 86 & 51.4 & 151 & 0.8 & $\mathrm{U}$ & 0.5 & $\mathrm{U}$ \\
\hline OU-B Marine (500-ft grid) & OUBM-G 11 & No & 89 & 50.0 & 147 & 1.1 & $\mathrm{~J}$ & 1.9 & $\mathrm{~J}$ \\
\hline OU-B Marine (500-ft grid) & OUBM-G 12 & No & 61 & 41.0 & 133 & 1.6 & $\mathrm{~J}$ & 0.1 & $\mathrm{U}$ \\
\hline OU-B Marine (500-ft grid) & OUBM-G 13 & No & 110 & 83.8 & 178 & 1.6 & $\mathrm{~J}$ & 1.2 & $\mathrm{~J}$ \\
\hline OU-B Marine (500-ft grid) & OUBM-G & No & 95 & 70.4 & 171 & 0.9 & $\mathrm{U}$ & 0.2 & $\mathrm{U}$ \\
\hline OU-B Marine (500-ft grid) & OUBM-G & No & 78 & 44.8 & 148 & 2.4 & $\mathrm{~J}$ & 0.0 & $\mathrm{U}$ \\
\hline OU-B Marine (500-ft grid) & OUBM-G & No & 68 & 46.2 & 142 & 1.8 & $\mathrm{~J}$ & 0.9 & $\mathrm{U}$ \\
\hline OU-B Marine (500-ft grid) & OUBM-G & No & 94 & 59.3 & 161 & 0.1 & $\mathrm{U}$ & 1.4 & J \\
\hline OU-B Marine (500-ft grid) & OUBM-G & No & 77 & 50.2 & 133 & ND & & 0.1 & $\mathrm{U}$ \\
\hline OU-B Marine (500-ft grid) & OUBM-G 19 & No & 84 & 48.8 & 153 & 1.4 & $\mathrm{~J}$ & 1.0 & $\mathrm{~J}$ \\
\hline OU-B Marine (500-ft grid) & OUBM-G & No & 90 & 64.8 & 154 & 0.7 & $\mathrm{U}$ & 0.8 & $\mathrm{U}$ \\
\hline
\end{tabular}


Table 9. (continued)

\begin{tabular}{|c|c|c|c|c|c|c|c|c|c|}
\hline \multirow{4}{*}{$\begin{array}{r}\text { Location Description } \\
\text { SQS } \\
\text { MCUL }\end{array}$} & \multirow{2}{*}{$\begin{array}{c}\text { Station or OUB } \\
\text { Grid Cell }\end{array}$} & \multirow{2}{*}{$\begin{array}{c}\text { Confirm } \\
\text { - atory } \\
\text { Sample? }\end{array}$} & \multicolumn{7}{|c|}{ Definitive ${ }^{\text {a }}$ Concentration (mg/kg dry weight) } \\
\hline & & & $\mathrm{Cu}$ & $\mathbf{P b}$ & Zn & $C d^{b}$ & & $\mathbf{A g}^{\mathbf{b}}$ & \\
\hline & & & 390 & 450 & 410 & 5.1 & & 6.1 & \\
\hline & & & 390 & 530 & 960 & 6.7 & & 6.1 & \\
\hline OU-B Marine (500-ft grid) & OUBM-G & No & 108 & 51.0 & 145 & ND & & ND & \\
\hline OU-B Marine (500-ft grid) & OUBM-G & No & 109 & 78.6 & 169 & 1.9 & $\mathrm{~J}$ & 0.5 & $\mathrm{U}$ \\
\hline OU-B Marine (500-ft grid) & OUBM-G & No & 128 & 74.1 & 168 & 1.6 & $\mathrm{~J}$ & 1.2 & $\mathrm{~J}$ \\
\hline OU-B Marine (500-ft grid) & OUBM-G & No & 161 & 72.6 & 206 & 1.5 & $\mathrm{~J}$ & ND & \\
\hline OU-B Marine (500-ft grid) & OUBM-G & No & 191 & 134 & 273 & 2.2 & $\mathrm{~J}$ & 2.2 & $\mathrm{~J}$ \\
\hline OU-B Marine (500-ft grid) & OUBM-G & No & 197 & 111 & 228 & 1.4 & $\mathrm{~J}$ & 1.9 & $\mathrm{~J}$ \\
\hline OU-B Marine (500-ft grid) & OUBM-G & No & 116 & 68.0 & 173 & 0.3 & $\mathrm{U}$ & ND & \\
\hline OU-B Marine (500-ft grid) & OUBM-G & No & 174 & 69.7 & 210 & 0.6 & $\mathrm{U}$ & 0.6 & $\mathrm{U}$ \\
\hline OU-B Marine (500-ft grid) & OUBM-G & No & 209 & 170 & 253 & 2.3 & $\mathrm{~J}$ & 1.1 & $\mathrm{~J}$ \\
\hline OU-B Marine (500-ft grid) & OUBM-G & No & 152 & 81.8 & 192 & 2.1 & $\mathrm{~J}$ & 1.6 & $\mathrm{~J}$ \\
\hline OU-B Marine (500-ft grid) & OUBM-G & No & 116 & 73.8 & 166 & 1.0 & $\mathrm{~J}$ & 0.7 & $\mathrm{U}$ \\
\hline OU-B Marine (500-ft grid) & OUBM-G & No & 121 & 67.1 & 172 & 0.6 & $\mathrm{U}$ & 0.1 & $\mathrm{U}$ \\
\hline OU-B Marine (500-ft grid) & OUBM-G & No & 193 & 106 & 256 & 1.8 & $\mathrm{~J}$ & 0.6 & $\mathrm{U}$ \\
\hline OU-B Marine (500-ft grid) & OUBM-G & Yes & 171 & 132 & 391 & 1.51 & & 2.38 & \\
\hline OU-B Marine (500-ft grid) & OUBM-G & No & 111 & 70.5 & 155 & 0.3 & $\mathrm{U}$ & 0.1 & $\mathrm{U}$ \\
\hline OU-B Marine (500-ft grid) & OUBM-G & Yes & 150 & 86.3 & 185 & 1.09 & & 0.838 & \\
\hline OU-B Marine (500-ft grid) & OUBM-G & No & 139 & 91.6 & 207 & ND & & 0.6 & $\mathrm{U}$ \\
\hline OU-B Marine (500-ft grid) & OUBM-G & No & 137 & 105 & 207 & 0.1 & $\mathrm{U}$ & 0.6 & $\mathrm{U}$ \\
\hline OU-B Marine (500-ft grid) & OUBM-G & Yes & 173 & 128 & 288 & 1.39 & & 1.11 & \\
\hline OU-B Marine (500-ft grid) & OUBM-G & Yes & 135 & 84.9 & 208 & 1.33 & & 0.709 & \\
\hline OU-B Marine (500-ft grid) & OUBM-G & No & 125 & 77.4 & 199 & 0.8 & $\mathrm{U}$ & 0.6 & $\mathrm{U}$ \\
\hline OU-B Marine (500-ft grid) & OUBM-G & Yes & 180 & 77.8 & 188 & 1.06 & & 0.688 & \\
\hline OU-B Marine (500-ft grid) & OUBM-G & Yes & 155 & 74.3 & 241 & 1.12 & & 0.588 & \\
\hline OU-B Marine (500-ft grid) & OUBM-G & No & 118 & 76.6 & 182 & 0.0 & $\mathrm{U}$ & 0.9 & $\mathrm{U}$ \\
\hline OU-B Marine (500-ft grid) & OUBM-G & Yes & 154 & 90.0 & 238 & 1.44 & & 0.74 & \\
\hline OU-B Marine (500-ft grid) & OUBM-G & Yes & 142 & 155 & $428^{e}$ & 0.621 & & 0.495 & \\
\hline OU-B Marine (500-ft grid) & OUBM-G & No & 117 & 73.8 & 199 & 0.3 & $\mathrm{U}$ & 0.4 & $\mathrm{U}$ \\
\hline OU-B Marine (500-ft grid) & OUBM-G & No & 97 & 56.5 & 152 & ND & & 1.2 & $\mathrm{~J}$ \\
\hline OU-B Marine (500-ft grid) & OUBM-G & No & 209 & 180 & 366 & 0.3 & $\mathrm{U}$ & 0.9 & $\mathrm{U}$ \\
\hline OU-B Marine (500-ft grid) & OUBM-G & No & 129 & 62.8 & 182 & 0.2 & $\mathrm{U}$ & ND & \\
\hline OU-B Marine (500-ft grid) & OUBM-G & No & 89 & 58.5 & 158 & ND & & 0.4 & $\mathrm{U}$ \\
\hline OU-B Marine (500-ft grid) & OUBM-G & Yes & 398 & 279 & 785 & 1.30 & & 1.07 & \\
\hline OU-B Marine (500-ft grid) & OUBM-G & No & 103 & 73.9 & 181 & 0.8 & $\mathrm{U}$ & ND & \\
\hline OU-B Marine (500-ft grid) & OUBM-G 54 & No & 94 & 66.7 & 171 & 1.5 & $\mathrm{~J}$ & 1.7 & $\mathrm{~J}$ \\
\hline OU-B Marine (500-ft grid) & OUBM-G 55 & No & 190 & 109 & 282 & 2.2 & $\mathrm{~J}$ & ND & \\
\hline OU-B Marine (500-ft grid) & OUBM-G & Yes & 194 & 105 & 324 & 1.54 & & 0.691 & \\
\hline OU-B Marine (500-ft grid) & OUBM-G & No & 140 & 145 & 268 & 1.2 & $\mathrm{~J}$ & ND & \\
\hline OU-B Marine (500-ft grid) & OUBM-G & No & 99 & 67.9 & 171 & 1.1 & $\mathrm{~J}$ & 0.3 & $\mathrm{U}$ \\
\hline OU-B Marine (500-ft grid) & OUBM-G & Yes & 272 & 439 & 736 & 3.17 & & 0.712 & \\
\hline OU-B Marine (500-ft grid) & OUBM-G & Yes & 200 & 180 & 1480 & 2.01 & & 0.642 & \\
\hline OU-B Marine (500-ft grid) & OUBM-G & No & 103 & 280 & 286 & 0.1 & $\mathrm{U}$ & 0.1 & $\mathrm{U}$ \\
\hline OU-B Marine (500-ft grid) & OUBM-G & No & 92 & 79.6 & 178 & 1.4 & $\mathrm{~J}$ & 0.2 & $\mathrm{U}$ \\
\hline OU-B Marine (500-ft grid) & OUBM-G & No & 276 & 180 & 369 & 0.0 & $\mathrm{U}$ & 0.0 & $\mathrm{U}$ \\
\hline OU-B Marine (500-ft grid) & OUBM-G & Yes & 230 & 209 & 425 & 1.85 & & 0.777 & \\
\hline
\end{tabular}


Table 9. (continued)

\begin{tabular}{|c|c|c|c|c|c|c|c|c|c|}
\hline \multirow{4}{*}{$\begin{array}{r}\text { Location Description } \\
\text { SQS } \\
\text { MCUL } \\
\end{array}$} & \multirow{2}{*}{$\begin{array}{c}\text { Station or OUB } \\
\text { Grid Cell }\end{array}$} & \multirow{2}{*}{$\begin{array}{c}\text { Confirm- } \\
\text { atory } \\
\text { Sample? }\end{array}$} & \multicolumn{7}{|c|}{ Definitive $^{\mathrm{a}}$ Concentration (mg/kg dry weight) } \\
\hline & & & $\mathbf{C u}$ & $\mathbf{P b}$ & $\mathbf{Z n}$ & $\mathbf{C d}^{\mathbf{b}}$ & & $\mathbf{A g}^{\mathbf{b}}$ & \\
\hline & & & 390 & 450 & 410 & 5.1 & & 6.1 & \\
\hline & & & 390 & 530 & 960 & 6.7 & & 6.1 & \\
\hline OU-B Marine (500-ft grid) & OUBM-G & No & 167 & 116 & 295 & ND & & 1.1 & $\mathrm{~J}$ \\
\hline OU-B Marine (500-ft grid) & OUBM-G 66 & Yes & 227 & 159 & 428 & 1.71 & & 0.547 & \\
\hline OU-B Marine (500-ft grid) & OUBM-G 67 & Yes & 710 & 204 & 547 & 1.53 & & 1.03 & \\
\hline OU-B Marine (500-ft grid) & OUBM-G 68 & Yes & 217 & 144 & 526 & 1.72 & & 0.762 & \\
\hline OU-B Marine (500-ft grid) & OUBM-G & No & 109 & 82.9 & 188 & 1.2 & $\mathrm{~J}$ & 0.1 & U \\
\hline OU-B Marine (500-ft grid) & OUBM-G & No & 96 & 70.8 & 170 & 0.3 & $\mathrm{U}$ & 1.9 & $\mathrm{~J}$ \\
\hline OU-B Marine (500-ft grid) & OUBM-G 71 & No & 65 & 43.4 & 113 & ND & & ND & \\
\hline Outside OU B (1500-ft grid) & OOUB-G & Yes & 102 & 198 & 547 & 0.479 & & 0.175 & \\
\hline Outside OU B (1500-ft grid) & OOUB-G & No & 27 & 19.9 & 88 & 0.1 & $\mathrm{U}$ & 0.6 & $\mathrm{U}$ \\
\hline Outside OU B (1500-ft grid) & OOUB-G & No & 46 & 20.7 & 102 & 0.1 & $\mathrm{U}$ & 1.0 & $\mathrm{~J}$ \\
\hline Outside OU B (1500-ft grid) & OOUB-G & No & 24 & 18.2 & 101 & 0.2 & $\mathrm{U}$ & 0.6 & $\mathrm{U}$ \\
\hline Outside OU B (1500-ft grid) & OOUB-G & No & 102 & 50.0 & 153 & 1.6 & $\mathrm{~J}$ & 1.2 & $\mathrm{~J}$ \\
\hline Outside OU B (1500-ft grid) & OOUB-G & No & 108 & 63.3 & 165 & 1.4 & $\mathrm{~J}$ & 1.3 & $\mathrm{~J}$ \\
\hline Outside OU B (1500-ft grid) & OOUB-G & Yes & 130 & 72.3 & 177 & 2.47 & & 1.35 & \\
\hline Outside OU B (1500-ft grid) & OOUB-G & No & 141 & 75.6 & 167 & 1.1 & $\mathrm{~J}$ & 0.7 & $\mathrm{U}$ \\
\hline Outside OU B (1500-ft grid) & OOUB-G & No & 120 & 66.8 & 177 & 0.9 & $\mathrm{U}$ & 0.3 & $\mathrm{U}$ \\
\hline Outside OU B (1500-ft grid) & OOUB-G & No & 109 & 72.4 & 166 & 0.5 & $\mathrm{U}$ & 0.1 & $\mathrm{U}$ \\
\hline Outside OU B (1500-ft grid) & OOUB-G & No & 118 & 72.1 & 157 & 0.5 & $\mathrm{U}$ & 0.2 & $\mathrm{U}$ \\
\hline Outside OU B (1500-ft grid) & OOUB-G & No & 42 & 25.2 & 100 & $\mathrm{ND}$ & & 0.3 & $\mathrm{U}$ \\
\hline Outside OU B (1500-ft grid) & OOUB-G 13 & Yes & 116 & 83.8 & 164 & 1.30 & & 0.971 & \\
\hline Outside OU B (1500-ft grid) & OOUB-G & No & 100 & 66.5 & 187 & ND & & 0.7 & $\mathrm{U}$ \\
\hline Outside OU B (1500-ft grid) & OOUB-G & No & 109 & 64.4 & 160 & 2.6 & $\mathrm{~J}$ & 1.0 & $\mathrm{~J}$ \\
\hline Outside OU B (1500-ft grid) & OOUB-G & No & 105 & 63.6 & 184 & 1.3 & $\mathrm{~J}$ & ND & \\
\hline Outside OU B (1500-ft grid) & OOUB-G & No & 103 & 75.8 & 161 & 1.7 & $\mathrm{~J}$ & 0.8 & $\mathrm{U}$ \\
\hline Outside OU B (1500-ft grid) & OOUB-G & Yes & 117 & 75.1 & 159 & 1.04 & & 0.943 & \\
\hline Outside OU B (1500-ft grid) & OOUB-G & No & 95 & 71.5 & 166 & 1.2 & $\mathrm{~J}$ & 1.7 & $\mathrm{~J}$ \\
\hline Outside OU B (1500-ft grid) & OOUB-G & No & 85 & 54.9 & 143 & 0.2 & $\mathrm{U}$ & 2.6 & $\mathrm{~J}$ \\
\hline Outside OU B (1500-ft grid) & OOUB-G & Yes & 102 & 71.8 & 159 & 1.32 & & 0.715 & \\
\hline Outside OU B (1500-ft grid) & OOUB-G & No & 88 & 71.3 & 154 & 1.8 & $\mathrm{~J}$ & 0.8 & $\mathrm{U}$ \\
\hline Outside OU B (1500-ft grid) & OOUB-G & No & 84 & 50.6 & 138 & 0.3 & $\mathrm{U}$ & ND & \\
\hline Outside OU B (1500-ft grid) & OOUB-G & Yes & 107 & 72.0 & 162 & 0.80 & & 0.705 & \\
\hline Outside OU B (1500-ft grid) & OOUB-G & No & 81 & 52.6 & 147 & 0.3 & $\mathrm{U}$ & ND & \\
\hline Outside OU B (1500-ft grid) & OOUB-G & Yes & 12 & 8.5 & 45 & 0.193 & & 0.042 & \\
\hline Outside OU B (1500-ft grid) & OOUB-G & Yes & 73 & 55.4 & 128 & 1.04 & & 0.417 & \\
\hline Outside OU B (1500-ft grid) & OOUB-G & Yes & 76 & 58.4 & 141 & 0.778 & & 0.50 & \\
\hline Outside OU B (1500-ft grid) & OOUB-G & No & 56 & 46.0 & 137 & 0.4 & $\mathrm{U}$ & 0.5 & $\mathrm{U}$ \\
\hline Outside OU B (1500-ft grid) & OOUB-G & No & 14 & 17.5 & 87 & ND & & ND & \\
\hline Outside OU B (1500-ft grid) & OOUB-G & No & 84 & 64.7 & 160 & 0.4 & $\mathrm{U}$ & 0.9 & $\mathrm{U}$ \\
\hline Outside OU B (1500-ft grid) & OOUB-G & No & 59 & 50.3 & 134 & ND & & & $\mathrm{U}$ \\
\hline
\end{tabular}
a. Definitive concentrations are ICP-MS result for confirmatory samples; estimated by linear regression for non- confirmatory samples.
b. Ag and Cd values are GFAA or ICP-MS result for confirmatory samples; XRF result for non-confirmatory samples.
c. J XRF result estimated above method detection limit but below quantitation limit.
d. U XRF result less than method detection limit.
e. Bold type in highlighted box indicates definitive result greater than SQS. 


\subsection{DISCUSSION AND CONCLUSIONS}

The Metals Verification Study was conducted to verify the present sediment metals concentrations in Sinclair and Dyes Inlets, where significant cleanup and source control actions have been implemented since the data were collected that led to placing several segments of those water bodies on the 303(d) list of impaired water bodies. The definitive metal concentrations are shown relative to Washington SQS and MCUL for $\mathrm{Cu}, \mathrm{Pb}$, and $\mathrm{Zn}$ in Figure 17. The vertical dashed lines indicate the major geographic divisions of outside Sinclair Inlet (Stations MVS-01 through MVS-59), inside Sinclair Inlet OU B Marine (OUBM-G01 through G71), and inside Sinclair Inlet but outside OU B Marine (OOUB-G01 through OOUB-G32).

Outside Sinclair Inlet, all Ag, Cd, Cu, Pb, and Zn concentrations were below Washington state SQS and MCUL (Figure 18); these sediment metals concentrations do not appear to be significant contributors to water quality impairment outside Sinclair Inlet, including Ostrich Bay in southern Dyes Inlet. Inside Sinclair Inlet, concentrations of Ag and Cd were below SQS in all sediment samples; therefore, the present-day data do not support 303(d) listing based on these metals in sediment. Present-day $\mathrm{Cu}, \mathrm{Pb}$, and Zn data also show that sediment metals concentrations are substantially lower than prior to cleanup and capping, with only the following samples exceeding state SQS or MCUL (Figure 19):

- Measured or predicted ICP-MS concentrations of Cu exceeded the SQS and MCUL in only 2 of the 103 samples, the composites representing OUBM Grids 52 and 67 (map Figure 14; chemistry results Figure 17).

- Measured or predicted ICP-MS concentrations of Zn exceeded the SQS in 6 of the 103 samples and the MCUL in just 1. Except for OOUB Grid 1 at Gorst, all of the samples were located in OU B Marine near PSNS piers or stormwater outfalls. OU-B continues to be managed and monitored under Superfund, and part of the source control efforts there includes stormwater monitoring and improvements to PSNS stormwater catchment systems. Zn at Gorst is likely due to nonpoint runoff sources as well as transport and deposition.

The primary outcome of the study was a nonstatistical comparison of target metal concentrations with Washington state SQS and MCUL, but the sampling and analysis design was intended to reduce uncertainty associated with the target measurements. The chance of false negatives (samples in which true metal concentration exceeds MCUL but measured concentration is less than MCUL) was limited by 1) increased sampling density where concentrations are likely to exceed SQS; 2) selecting methods and setting quality control limits to minimize analytical error; and 3) comparing screening level concentrations to $90 \%$ MCUL. The chance of false positives (samples in which true metal concentration is below MCUL but measured concentration exceeds MCUL) was also limited by appropriate sampling density, analytical method selection, and analytical quality control. 

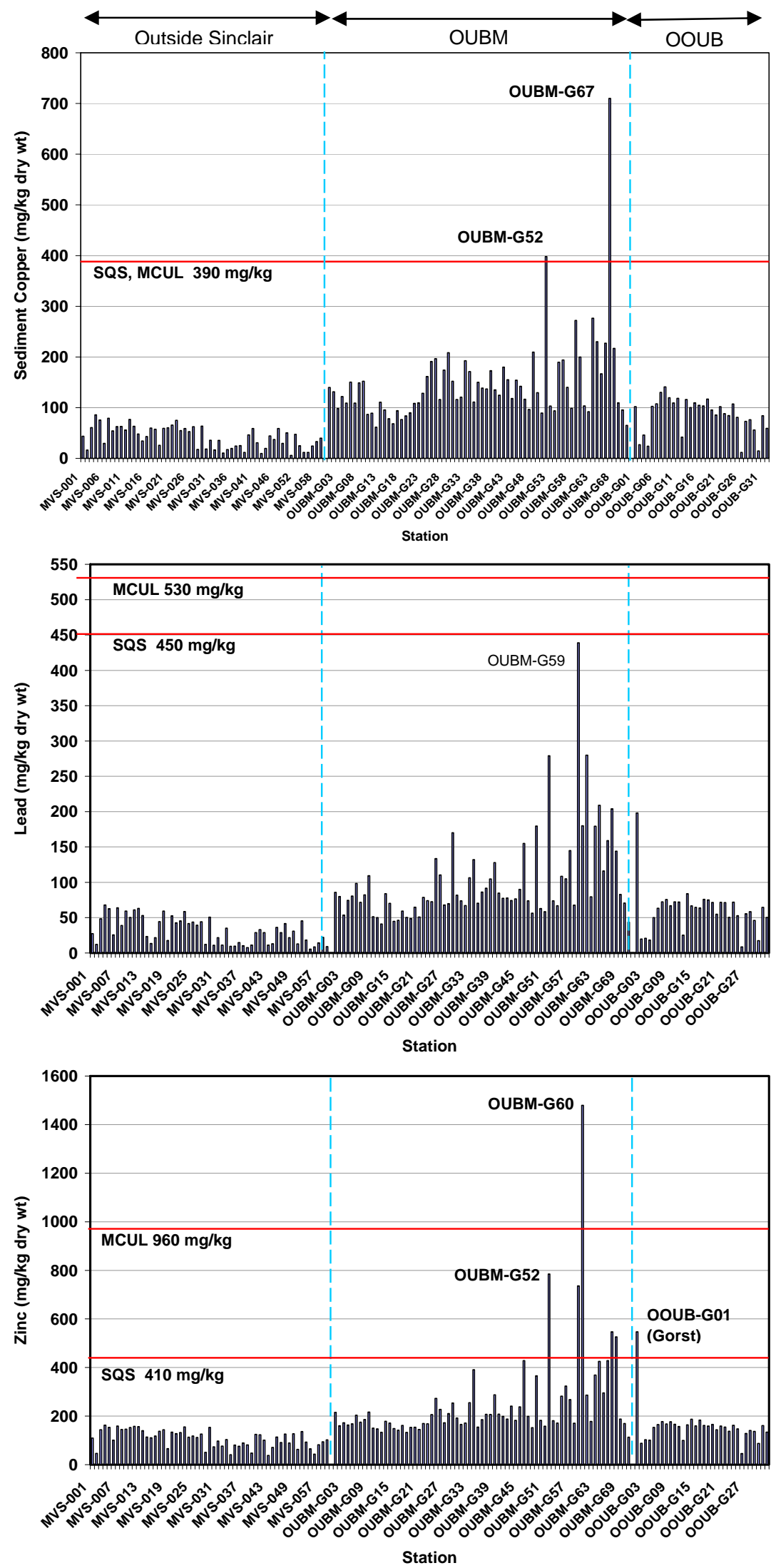

Figure 17. Definitive sediment Cu (top), Pb (middle), and Zn (bottom) ENVVEST Metals Verification Study 


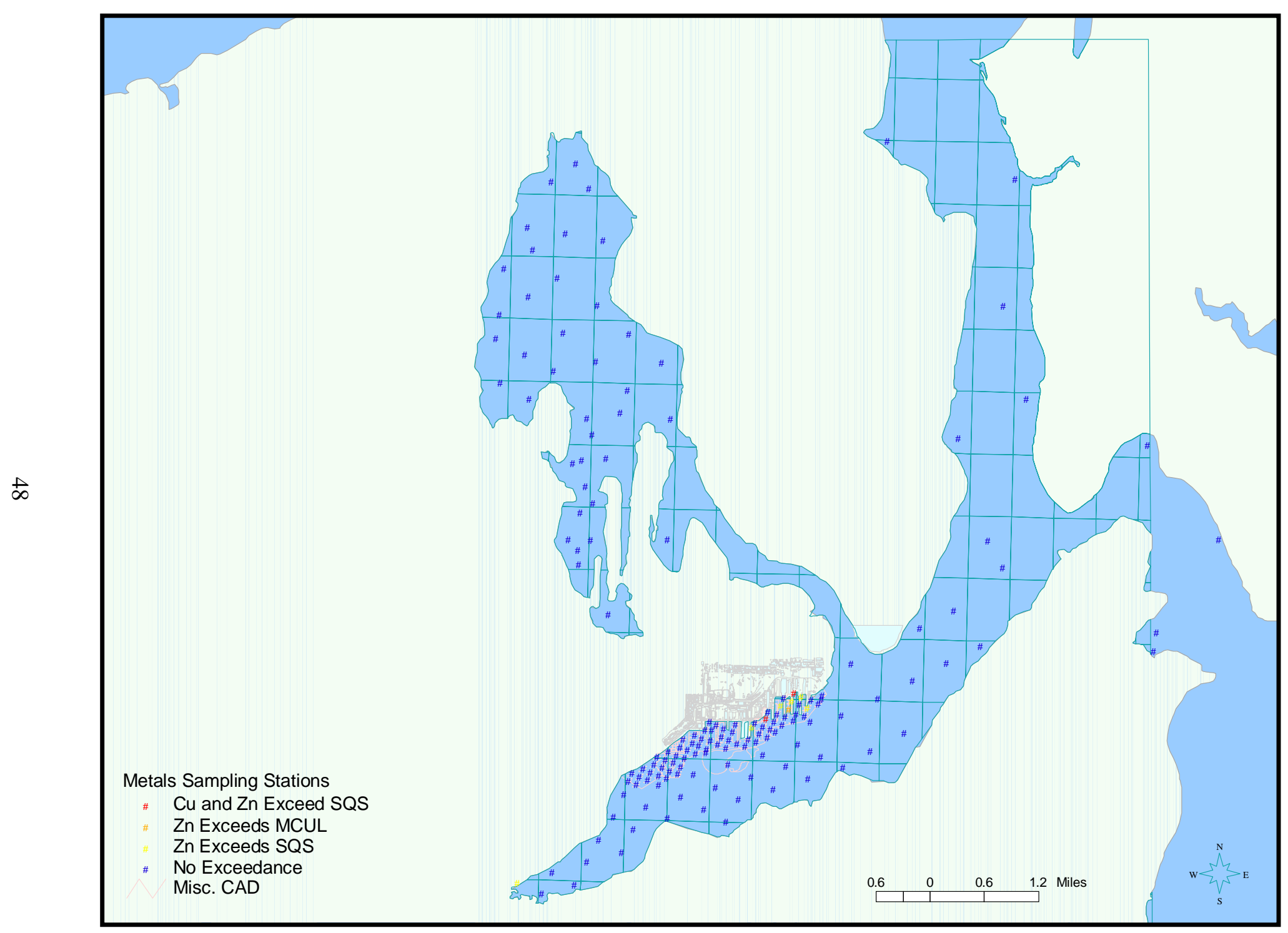

Figure 18. Locations where definitive concentrations exceed SQS or MCUL in entire Metals Verification Study area 


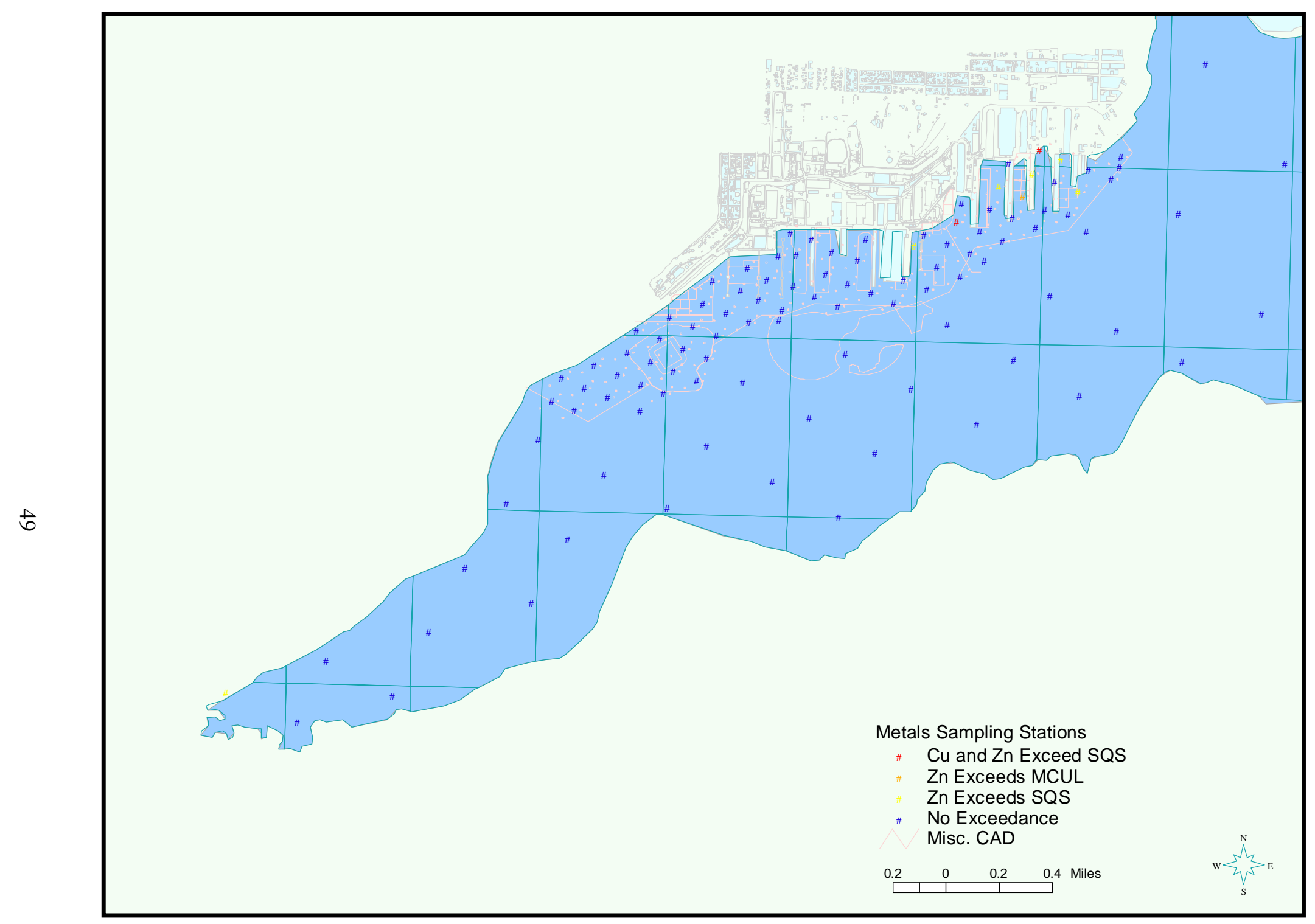

Figure 19. Locations where definitive concentrations exceed SQS or MCUL in Sinclair Inlet 
The XRF method with 20\% confirmatory analyses by ICP-MS provides cost-effective alternative of screening a large number of samples for $\mathrm{Cu}, \mathrm{Pb}$, and $\mathrm{Zn}$. However, samples with highest XRF concentrations are always recommended for confirmatory analysis because there are fewer data to support the linear relationship between methods.

In conclusion, the Metals Verification Study was successful in showing that $\mathrm{Ag}, \mathrm{Cd}$, and $\mathrm{Pb}$ concentrations met Washington SQS in all sediment throughout Sinclair and Dyes Inlets. SQS for Cu and Zn were met in all but a few samples concentrated near known sources where pollution source controls, stormwater monitoring, and sediment monitoring are in place. Where As in sediment was measured (at least 3 samples per 303(d)-listed segment), concentrations also met SQS throughout Sinclair and Dyes Inlets.

The Metals Verification Study data indicate that sediment quality in Sinclair Inlet has improved markedly since implementation of cleanup and source control actions; these 2003 data do not support 303(d) listing for most if not all the target metals in this study. The Metals Verification Study also supports SinclairDyes Inlet watershed and water quality models by providing spatially distributed sediment metals data collected throughout the Sinclair-Dyes Inlet water quality modeling domain, including model initialization stations in eastern Rich Passage and northern Port Orchard Passage. 


\subsection{REFERENCES}

Diefenderfer, H. L., N. P. Kohn, E. A. Crecelius, R. K. Johnston, and G. M. Sherrell. 2003. Technical Approach to Address Section 303(d) Listings for Metals in Sinclair and Dyes Inlets. May 2003. Prepared for Project ENVVEST, Bremerton, Washington.

Ecology (Washington State Department of Ecology). 2003. The 303(d) List of Impaired and Threatened Waterbodies: 1998 List - By Water Resource Inventory Areas (WRIAs). http://www.ecy.wa.gov/programs/wq/303d/1998/1998 by wrias.html

EPA (U.S. Environmental Protection Agency). 1998. Method 6200: Field Portable X-ray Fluorescence Spectrometry for the Determination of Elemental Concentrations in Soil and Sediment. U.S.

Environmental Protection Agency, Office of Solid Waste and Emergency Response, Washington, DC. http://www.epa.gov/SW-846/pdfs/6200.pdf

EPA (U. S. Environmental Protection Agency). 2001. Methods for Collection, Storage and Manipulation of Sediments for Chemical and Toxicological Analyses: Technical Manual. EPA-823-B01-002. U. S. Environmental Protection Agency, Office of Water, Washington, DC. http://www.epa.gov/waterscience/cs/collectionmanual.pdf

Katz, C.N., P.L. Noble, D.B. Chadwick, B. Davidson, and R.D. Gauthier. 1999. Sinclair Inlet Water Quality Assessment, Draft. Puget Sound Wastewater Technology Evaluation Research Project.

Kohn, N. P., M. C. Miller, H. L. Diefenderfer, R. K. Johnston, G. M. Sherrell, and B. Beckwith. 2003. Sampling and Analysis Plan, Sediment Metals Verification Study for Sinclair and Dyes Inlet, Washington. August 19, 2003. Prepared for Puget Sound Naval Shipyard \& Intermediate Maintenance Facility Project ENVVEST, Bremerton, Washington.

Miller M.C., R.K. Johnston, E.A. Crecelius, J.M. Brandenberger, J. Leather, and J. Guerrero. 2003. Contaminant Mass Balance for Sinclair and Dyes Inlets, Puget Sound Washington. PNNL-SA-38987, Pacific Northwest National Laboratory, Richland, Washington.

PSEP (Puget Sound Estuary Program). 1997. Recommended Guidelines for Sampling Marine Sediment, Water Column, and Tissue in Puget Sound. U.S. Environmental Protection Agency Region X, Office of Puget Sound, and Puget Sound Water Quality Authority, Seattle, Washington. April 1997.

URS (URS Greiner, Inc.). 2002a. Final Remedial Investigation Report, Operable Unit B Marine, Bremerton Naval Complex, Washington. Prepared by URS Greiner, Inc. for U.S. Navy, Engineering Field Activity, Northwest, Poulsbo, Washington.

URS (URS Greiner, Inc.). 2002b. Draft Long Term Monitoring Plan, Operable Unit B Marine, Bremerton Naval Complex, Washington. Appendix B, 12/06/02. Prepared by URS Greiner, Inc. for U.S. Navy, Engineering Field Activity, Northwest, Poulsbo, Washington.

U.S. Navy. 2000a. Final Record of Decision for Bremerton Naval Complex, Operable Unit B Marine. U.S. Navy, Engineering Field Activity, Northwest Date: 06/06/00 Contract No. N62474-89-D-9295, CTO 0131, Bremerton, Washington. http://yosemite.epa.gov/r10/cleanup.nsf/webpage/Superfund+(CERCLA)\#sites

U.S. Navy 2000b. Record of Decision for Jackson Park Housing Complex/Naval Hospital Bremerton, Operable Unit 1, Sites 101, 101-A, 103, and 110, Bremerton, Washington.

U.S. Navy. 2003. Final Five-Year Review of Record of Decision Bremerton Naval Complex, Revision Number 0. U.S. Navy, Engineering Field Activity, Northwest Date: 10/17/02 Contract No. N44255-00D-2476 Delivery Order 0017.

http://yosemite.epa.gov/r10/cleanup.nsf/webpage/Superfund+(CERCLA)\#sites 
Washington State. 1995. Chapter 173-204 WAC Sediment Management Standards. http://www.ecy.wa.gov/pubs/wac173204/index.html. 
APPENDIX A

X-RAY FLUORESCENCE LABORATORY REPORT 

Sample Location: Puget Sound Naval Shipyard (Dyes Inlet, Port Orchard Passage, Rich Passage)

Sample Type: Marine sediment samples

Analysis: Metals by Energy Dispersive X-ray Fluorescence (EDXRF) Spectrometry

EDXRF Application: Soils Method (06/99) (Rapid Screening Method 1: wet samples)

Sample Date: 8/28/03 - 8/31/03,

Analysis Date: 9/8/03 - 9/16/03,

Analyst: Brian Ayers, SPAWARSYSCEN, San Diego: 619.553.1613

Analysis Location: SPAWARSYSCEN, San Diego (SSC SD), BS Bldg 111 Rm 236)

POC: Victoria Kirtay, SPAWARSYSCEN, San Diego: 619.553.1395; Brian Ayers, SPAWARSYSCEN, San Diego: 619.553 .1613

\section{Analytical Technique:}

X-Ray Fluorescence spectrometry is an analytical technique that can provide rapid, multi-element analysis of metals in sediment. Samples are exposed to $\mathrm{X}$-ray energy, which liberates electrons in the inner shell of metal atoms. As the outer electrons cascade towards the inner shells to fill the vacancies, energy is released (fluorescence). The fluorescing energy spectrum identifies the metals and the intensity is proportional to concentration.

\section{Analytical Instrument:}

Sediment samples will be analyzed using a QuanX EDXRF Spectrometer (Spectrace Instruments, Sunnyvale, CA). This instrument contains a Rh-anode $\mathrm{X}$-ray tube for primary generation of $\mathrm{X}$-rays $(4-50 \mathrm{kV})$ and a thermoelectrically cooled, solid-state Silicon(Lithium) detector. The $\mathrm{Si}(\mathrm{Li})$ detector provides spectral resolution which exceeds other solid-state detectors or gas-filled proportional detectors.

\section{Instrument Calibration:}

The QuanX EDXRF is calibrated using semi-standardless calibration (Fundamental Parameters).

Five standard reference materials (NIST 2704, 2709, 2710, 2711 and CNRC PACS-1) are used for standardization.

\section{Statistically-based Method Detection Limits (note: not field-based detection limits)}

The QuanX EDXRF was calibrated using semi-standardless calibration (Fundamental Parameters). Five standard reference materials (NIST 2704, 2709, 2710, 2711 and CRC PACS-1) were used for standardization. Detection limits were calculated from these standards as follows:

$\operatorname{LLD}=3\left(\right.$ sqrt $\left.\mathrm{N}_{\mathrm{b}}\right) *$ conc. $/ \mathrm{N}_{\mathrm{p}}$

where;

$\mathrm{N}_{\mathrm{b}}$ = the background counts

$\mathrm{N}_{\mathrm{p}}$ = the peak counts

conc. $=$ concentration of the analyte in the standard

IDL = Instrument Detection Limit (quartz)

MDL = Method Detection Limit (same as LLD, 3 sigma)

LOQ Limit of Quantitation (10 sigma) 


\begin{tabular}{lccc} 
& (quartz) & (3 sigma) & (10 sigma) \\
Analyte & IDL & MDL & LOQ \\
\hline Cl (\%) & NC & 0.083 & 0.277 \\
K (\%) & 0.009 & 0.021 & 0.070 \\
Ca (\%) & 0.006 & 0.014 & 0.047 \\
Ti (ppm) & 11 & 39 & 130 \\
Cr (ppm) & 4 & 11 & 36 \\
Mn (ppm) & 7 & 14 & 48 \\
Fe (\%) & 0.003 & 0.005 & 0.017 \\
Ni (ppm) & 14 & 17 & 55 \\
Cu (ppm) & 7 & 9 & 29 \\
Zn (ppm) & 5 & 8 & 27 \\
Se (ppm) & 1 & 2 & 5 \\
As (ppm) & 2 & 9 & 29 \\
Ag (ppm) & 1 & 1 & 3 \\
Cd (ppm) & 1 & 1 & 4 \\
Sn (ppm) & 1 & 1 & 4 \\
Ba (ppm) & 10 & 10 & 34 \\
Pb (ppm) & 3 & 4 & 15
\end{tabular}

\section{Sample Handling and Analysis:}

Sediment screening samples will be shipped to the destination laboratory by overnight courier at the end of each sampling day in coolers with blue ice.

Sediment screening samples will be shipped to the Space and Naval Warfare Systems Center San Diego (SSC San Diego).

For rapid screening analysis of sediment samples, five to ten grams of the wet sediment are placed in an XRF sample cup and covered with Mylar film.

Analysis time for each sample is 30 minutes.

The sample will be analyzed for a suite of metals (e.g., Cl, K, Ca, Ti, Fe, Mn, Ba, Cr, Cu, Zn, Pb, Se, As, Ag, Cd, Sn and Ni).

Results are calculated based on Fundamental Parameters (FP) calibration and are computed using FP algorithms.

Because XRF is a non-destructive technique, the samples can either be archived or used for other analyses.

\section{Data Quality (see EDXRFQAPP_0803.doc for details)}

\section{$\underline{\text { Accuracy }}$}

1. \% Recovery (used for accuracy measurement with SRMs)

2. Instrument Blank (analysis of ground quartz; results should not exceed MDL)

$\underline{\text { Precision }}$

1. RPD = Relative Percent Difference (used for comparison of field duplicate measurements)

2. $\mathrm{RSD}=$ Relative Standard Deviation (comparis (10 sigma) 
Project:

Samples Received:

Metals Reported:
ENVVEST Metals Verification Study (Project No. 43043)

9/4/2003

Fe, Cu, Pb, Zn, Cd, Ag

Rapid Screening Method: EDXRF (wet)

(concentrations in mg/kg, except where noted; not blank corrected)

\begin{tabular}{|c|c|c|c|c|c|c|c|c|c|c|c|c|c|c|c|c|c|}
\hline Sample ID & $\mathrm{Fe}(\%)$ & Stdev Q & $\mathrm{Cu}$ & Stdev & Q & $\mathrm{Pb}$ & Stdev & $\mathrm{Q}$ & $\mathrm{Zn}$ & Stdev & Q & $\mathrm{Cd}$ & Stdev & Q & $\mathrm{Ag}$ & Stdev & $\mathrm{Q}$ \\
\hline MVS-001-XRF & 2.092 & & 34 & & & 17 & & & 58 & & & 1.2 & & $\mathrm{~J}$ & 0.1 & & U \\
\hline MVS-002-XRF & 1.601 & & 18 & & $\mathrm{~J}$ & 9 & & $\mathrm{~J}$ & 38 & & & 2.3 & & $\mathrm{~J}$ & 0.3 & & $\mathrm{U}$ \\
\hline MVS-003-XRF & 2.071 & 0.024 & 46 & 3 & & 30 & 4 & & 84 & 4 & & 0.5 & 0.1 & $\mathrm{U}$ & 0.3 & 0.3 & $\mathrm{U}$ \\
\hline MVS-004-XRF & 2.192 & & 63 & & & 41 & & & 98 & & & ND & & $\mathrm{U}$ & 0.9 & & U \\
\hline MVS-005-XRF & 2.073 & & 56 & & & 38 & & & 91 & & & 0.6 & & $\mathrm{U}$ & 1.4 & & $\mathrm{~J}$ \\
\hline MVS-006-XRF & 1.884 & & 25 & & $\mathrm{~J}$ & 16 & & & 52 & & & 0.8 & & $\mathrm{U}$ & 0.4 & & $\mathrm{U}$ \\
\hline MVS-007-XRF & 2.208 & 0.035 & 57 & 5 & & 39 & 3 & & 97 & 1 & & 0.6 & 0.6 & $\mathrm{U}$ & 0.7 & 0.6 & $\mathrm{U}$ \\
\hline MVS-008-XRF & 2.078 & & 41 & & & 24 & & & 85 & & & ND & & $\mathrm{U}$ & 0.9 & & $\mathrm{U}$ \\
\hline MVS-009-XRF & 2.217 & & 47 & & & 36 & & & 85 & & & 0.7 & & $\mathrm{U}$ & 1.8 & & $\mathrm{~J}$ \\
\hline MVS-010-XRF & 2.073 & 0.014 & 47 & 1 & & 31 & 4 & & 91 & 3 & & 0.5 & 0.3 & $\mathrm{U}$ & 0.2 & 0.3 & $\mathrm{U}$ \\
\hline MVS-011-XRF & 2.164 & & 43 & & & 37 & & & 95 & & & 0.5 & & $\mathrm{U}$ & 0.8 & & $\mathrm{U}$ \\
\hline MVS-012-XRF & 2.246 & & 57 & & & 38 & & & 94 & & & 0.4 & & $\mathrm{U}$ & 0.6 & & U \\
\hline MVS-013-XRF & 2.239 & & 43 & & & 30 & & & 86 & & & 0.7 & & $\mathrm{U}$ & ND & & $\mathrm{U}$ \\
\hline MVS-014-XRF & 2.337 & & 37 & & & 15 & & & 62 & & & ND & & $\mathrm{U}$ & ND & & U \\
\hline MVS-015-XRF & 3.032 & 0.063 & 28 & 3 & $\mathrm{~J}$ & 9 & 1 & $\mathrm{~J}$ & 59 & 4 & & 0.0 & 0.0 & $\mathrm{U}$ & 0.4 & 0.4 & U \\
\hline MVS-016-XRF & 2.456 & 0.020 & 34 & 4 & & 14 & 1 & $\mathrm{~J}$ & 65 & 0 & & 0.7 & 0.4 & $\mathrm{U}$ & 0.1 & 0.1 & $\mathrm{U}$ \\
\hline MVS-017-XRF & 2.072 & & 45 & & & 27 & & & 79 & & & 0.3 & & $\mathrm{U}$ & 0.3 & & U \\
\hline MVS-018-XRF & 2.191 & & 44 & & & 36 & & & 84 & & & 1.5 & & $\mathrm{~J}$ & 0.9 & & U \\
\hline MVS-019-XRF & 1.523 & & 34 & & & 13 & & $\mathrm{~J}$ & 45 & & & 0.6 & & $\mathrm{U}$ & 0.1 & & U \\
\hline MVS-020-XRF & 2.053 & & 45 & & & 32 & & & 77 & & & ND & & $\mathrm{U}$ & ND & & $\mathrm{U}$ \\
\hline MVS-021-XRF & 2.002 & 0.038 & 46 & 4 & & 26 & 3 & & 72 & 7 & & 1.0 & 1.2 & $\mathrm{~J}$ & 1.3 & 0.2 & $\mathrm{~J}$ \\
\hline MVS-022-XRF & 2.207 & & 53 & & & 32 & & & 89 & & & 1.3 & & $\mathrm{~J}$ & ND & & U \\
\hline MVS-023-XRF & 2.160 & & 55 & & & 36 & & & 93 & & & 0.2 & & $\mathrm{U}$ & 0.5 & & U \\
\hline MVS-024-XRF & 2.140 & & 49 & & & 26 & & & 77 & & & ND & & $\mathrm{U}$ & 0.3 & & $\mathrm{U}$ \\
\hline MVS-025-XRF & 1.986 & 0.021 & 44 & 8 & & 28 & 3 & & 71 & 5 & & 0.7 & 0.6 & $\mathrm{U}$ & 0.5 & 0.4 & U \\
\hline MVS-026-XRF & 2.046 & & 47 & & & 30 & & & 73 & & & 0.4 & & $\mathrm{U}$ & 0.5 & & $\mathrm{U}$ \\
\hline MVS-027-XRF & 2.083 & 0.009 & 50 & 5 & & 30 & 5 & & 77 & 7 & & 0.8 & 0.5 & $\mathrm{U}$ & 0.3 & 0.5 & U \\
\hline MVS-028-XRF & 1.757 & & 22 & & $\mathrm{~J}$ & 12 & & $\mathrm{~J}$ & 46 & & & 0.7 & & $\mathrm{U}$ & ND & & $\mathrm{U}$ \\
\hline MVS-029-XRF & 2.123 & & 48 & & & 31 & & & 91 & & & 2.2 & & $\mathrm{~J}$ & ND & & $\mathrm{U}$ \\
\hline MVS-030-XRF & 1.506 & & 17 & & $\mathrm{~J}$ & 8 & & $\mathrm{~J}$ & 31 & & & 0.8 & & $\mathrm{U}$ & ND & & U \\
\hline MVS-031-XRF & 1.715 & & 29 & & & 14 & & $\mathrm{~J}$ & 49 & & & 1.3 & & $\mathrm{~J}$ & ND & & $\mathrm{U}$ \\
\hline MVS-032-XRF & 1.739 & & 16 & & $\mathrm{~J}$ & 8 & & $\mathrm{~J}$ & 33 & & & ND & & $\mathrm{U}$ & ND & & U \\
\hline MVS-033-XRF & 1.844 & & 29 & & & 22 & & & 53 & & & ND & & $\mathrm{U}$ & 1.3 & & $\mathrm{~J}$ \\
\hline MVS-034-XRF & 1.442 & & 16 & & $\mathrm{~J}$ & 7 & & $\mathrm{~J}$ & 29 & & & ND & & $\mathrm{U}$ & 0.1 & & $\mathrm{U}$ \\
\hline MVS-035-XRF & 1.542 & & 16 & & $\mathrm{~J}$ & 7 & & $\mathrm{~J}$ & 37 & & & 0.8 & & $\mathrm{U}$ & ND & & $\mathrm{U}$ \\
\hline MVS-036-XRF & 1.810 & & 18 & & $\mathrm{~J}$ & 10 & & $\mathrm{~J}$ & 33 & & & ND & & $\mathrm{U}$ & ND & & $\mathrm{U}$ \\
\hline MVS-037-XRF & 1.689 & & 21 & & $\mathrm{~J}$ & 7 & & $\mathrm{~J}$ & 43 & & & 1.6 & & $\mathrm{~J}$ & ND & & U \\
\hline MVS-038-XRF & 1.716 & & 22 & & $\mathrm{~J}$ & 6 & & $\mathrm{~J}$ & 38 & & & 0.6 & & $\mathrm{U}$ & ND & & $\mathrm{U}$ \\
\hline MVS-039-XRF & 1.786 & & 21 & & $\mathrm{~J}$ & 11 & & $\mathrm{~J}$ & 34 & & & 0.4 & & $\mathrm{U}$ & 1.0 & & $\mathrm{~J}$ \\
\hline MVS-040-XRF & 2.090 & & 36 & & & 18 & & & 70 & & & 0.7 & & $\mathrm{U}$ & ND & & U \\
\hline MVS-041-XRF & 2.153 & & 45 & & & 21 & & & 68 & & & ND & & $\mathrm{U}$ & ND & & U \\
\hline MVS-042-XRF & 1.966 & 0.039 & 25 & 2 & $\mathrm{~J}$ & 18 & 5 & & 52 & 2 & & 0.7 & 1.1 & $\mathrm{U}$ & 0.3 & 0.3 & $\mathrm{U}$ \\
\hline MVS-043-XRF & 1.550 & & 13 & & $\mathrm{~J}$ & 11 & & $\mathrm{~J}$ & 33 & & & 1.6 & & $\mathrm{~J}$ & 2.3 & & $\mathrm{~J}$ \\
\hline MVS-044-XRF & 1.396 & & 18 & & $\mathrm{~J}$ & 9 & & $\mathrm{~J}$ & 29 & & & ND & & $\mathrm{U}$ & 0.0 & & $\mathrm{U}$ \\
\hline MVS-045-XRF & 2.027 & 0.037 & 35 & 6 & & 23 & 1 & & 62 & 2 & & 0.5 & 0.5 & $\mathrm{U}$ & 0.2 & 0.4 & $\mathrm{U}$ \\
\hline MVS-046-XRF & 1.754 & & 30 & & & 18 & & & 44 & & & ND & & $\mathrm{U}$ & ND & & $\mathrm{U}$ \\
\hline MVS-047-XRF & 2.284 & & 55 & & & 22 & & & 72 & & & 1.5 & & $\mathrm{~J}$ & 0.4 & & $\mathrm{U}$ \\
\hline MVS-048-XRF & 2.021 & & 25 & & $\mathrm{~J}$ & 14 & & $\mathrm{~J}$ & 43 & & & 0.5 & & $\mathrm{U}$ & 1.7 & & $\mathrm{~J}$ \\
\hline MVS-049-XRF & 2.178 & & 39 & & & 19 & & & 72 & & & 1.1 & & $\mathrm{~J}$ & 1.2 & & $\mathrm{~J}$ \\
\hline MVS-050-XRF & 1.288 & & 9 & & $\mathrm{~J}$ & 9 & & $\mathrm{~J}$ & 24 & & $\mathrm{~J}$ & 0.4 & & $\mathrm{U}$ & 0.5 & & U \\
\hline MVS-052-XRF & 2.050 & 0.021 & 37 & 0 & & 28 & 2 & & 78 & 3 & & 2.3 & 0.5 & $\mathrm{~J}$ & 0.4 & 0.2 & $\mathrm{U}$ \\
\hline MVS-054-XRF & 1.670 & & 22 & & $\mathrm{~J}$ & 12 & & $\mathrm{~J}$ & 46 & & & 1.3 & & $\mathrm{~J}$ & 1.8 & & $\mathrm{~J}$ \\
\hline MVS-055-XRF & 1.161 & & 13 & & $\mathrm{~J}$ & 4 & & $\mathrm{~J}$ & 25 & & $\mathrm{~J}$ & 1.6 & & $\mathrm{~J}$ & 0.4 & & U \\
\hline MVS-056-XRF & 1.295 & & 14 & & $\mathrm{~J}$ & 6 & & $\mathrm{~J}$ & 28 & & & ND & & $\mathrm{U}$ & 0.9 & & $\mathrm{U}$ \\
\hline
\end{tabular}


Project:

Samples Received:

Metals Reported:
ENVVEST Metals Verification Study (Project No. 43043)

9/4/2003

Fe, Cu, Pb, Zn, Cd, Ag

Rapid Screening Method: EDXRF (wet)

(concentrations in mg/kg, except where noted; not blank corrected)

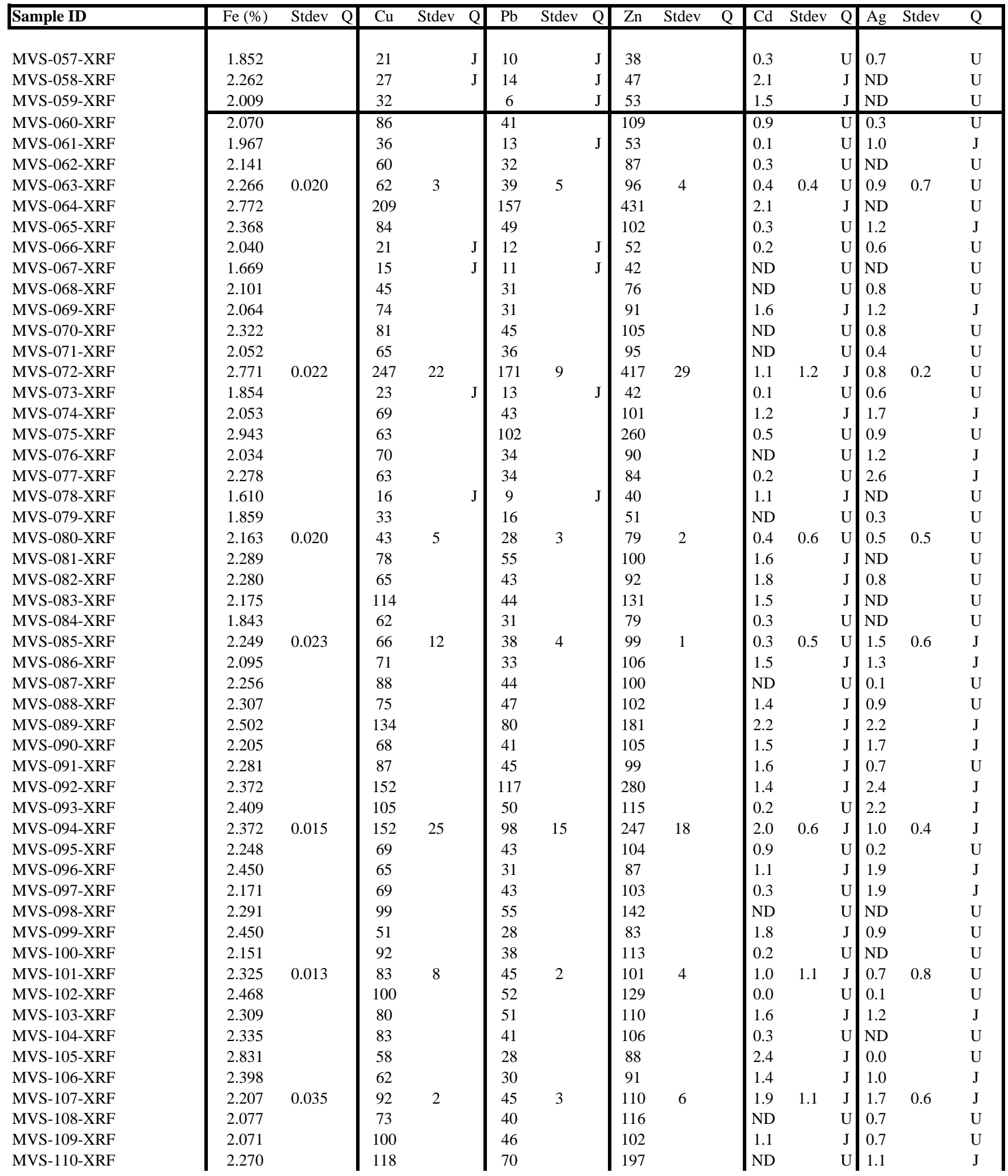


Project:

Samples Received:

Metals Reported:
ENVVEST Metals Verification Study (Project No. 43043)

9/4/2003

Fe, Cu, Pb, Zn, Cd, Ag

Rapid Screening Method: EDXRF (wet)

(concentrations in mg/kg, except where noted; not blank corrected)

\begin{tabular}{|c|c|c|c|c|c|c|c|c|c|c|c|c|c|c|}
\hline Sample ID & $\mathrm{Fe}(\%)$ & Stdev Q & $\mathrm{Cu}$ & Stdev Q & $\mathrm{Pb}$ & Stdev Q & $\mathrm{Zn}$ & Stdev Q & $\mathrm{Cd}$ & Stdev & Q & $\mathrm{Ag}$ & Stdev & $\mathrm{Q}$ \\
\hline MVS-111-XRF & 2.220 & & 100 & & 87 & & 177 & & 1.2 & & $\mathrm{~J}$ & ND & & U \\
\hline MVS-112-XRF & 2.441 & & 133 & & 65 & & 188 & & 2.2 & & $\mathrm{~J}$ & ND & & $\mathrm{U}$ \\
\hline MVS-113-XRF & 2.504 & & 133 & & 92 & & 286 & & 1.7 & & $\mathrm{~J}$ & 1.8 & & $\mathrm{~J}$ \\
\hline MVS-114-XRF & 2.308 & 0.018 & 85 & 4 & 46 & 4 & 113 & 6 & 0.0 & 0.0 & $\mathrm{U}$ & 0.9 & 0.6 & U \\
\hline MVS-115-XRF & 2.226 & & 106 & & 49 & & 148 & & 2.6 & & $\mathrm{~J}$ & ND & & $\mathrm{U}$ \\
\hline MVS-116-XRF & 2.256 & & 58 & & 34 & & 87 & & 2.4 & & $\mathrm{~J}$ & 0.7 & & $\mathrm{U}$ \\
\hline MVS-117-XRF & 2.444 & & 105 & & 56 & & 133 & & 0.4 & & $\mathrm{U}$ & 0.6 & & $\mathrm{U}$ \\
\hline MVS-118-XRF & 2.405 & & 84 & & 45 & & 125 & & 0.3 & & $\mathrm{U}$ & 0.4 & & $\mathrm{U}$ \\
\hline MVS-119-XRF & 2.383 & & 146 & & 102 & & 166 & & 2.3 & & $\mathrm{~J}$ & 1.1 & & $\mathrm{~J}$ \\
\hline MVS-120-XRF & 2.250 & & 78 & & 31 & & 85 & & ND & & $\mathrm{U}$ & ND & & U \\
\hline MVS-121-XRF & 2.447 & 0.011 & 108 & 19 & 66 & 2 & 138 & 8 & 0.0 & 0.1 & $\mathrm{U}$ & 1.3 & 0.9 & $\mathrm{~J}$ \\
\hline MVS-122-XRF & 2.411 & & 78 & & 43 & & 108 & & 1.1 & & $\mathrm{~J}$ & 1.1 & & $\mathrm{~J}$ \\
\hline MVS-123-XRF & 2.207 & & 94 & & 48 & & 96 & & 1.7 & & $\mathrm{~J}$ & 1.0 & & $\mathrm{~J}$ \\
\hline MVS-124-XRF & 2.279 & & 135 & & 64 & & 168 & & 1.8 & & $\mathrm{~J}$ & 0.6 & & U \\
\hline MVS-125-XRF & 2.475 & & 106 & & 59 & & 129 & & 1.0 & & $\mathrm{~J}$ & 2.4 & & $\mathrm{~J}$ \\
\hline MVS-126-XRF & 2.481 & & 97 & & 63 & & 131 & & 0.1 & & $\mathrm{U}$ & 0.6 & & $\mathrm{U}$ \\
\hline MVS-127-XRF & 2.436 & & 131 & & 66 & & 173 & & 0.7 & & $\mathrm{U}$ & 1.4 & & $\mathrm{~J}$ \\
\hline MVS-128-XRF & 2.324 & & 138 & & 66 & & 147 & & 1.4 & & $\mathrm{~J}$ & 1.9 & & $\mathrm{~J}$ \\
\hline MVS-129-XRF & 2.162 & & 192 & & 107 & & 253 & & 0.0 & & $\mathrm{U}$ & 0.0 & & U \\
\hline MVS-130-XRF & 2.125 & 0.031 & 122 & 12 & 42 & 2 & 134 & 2 & 0.6 & 1.1 & $\mathrm{U}$ & 0.6 & 0.9 & $\mathrm{U}$ \\
\hline MVS-131-XRF & 2.301 & & 63 & & 31 & & 89 & & 0.8 & & $\mathrm{U}$ & 0.5 & & U \\
\hline MVS-132-XRF & 2.128 & & 129 & & 67 & & 558 & & 1.2 & & $\mathrm{~J}$ & 1.4 & & $\mathrm{~J}$ \\
\hline MVS-133-XRF & 2.123 & & 79 & & 48 & & 103 & & 1.9 & & $\mathrm{~J}$ & 0.5 & & U \\
\hline MVS-134-XRF & 2.290 & & 122 & & 61 & & 166 & & 1.5 & & $\mathrm{~J}$ & 0.5 & & $\mathrm{U}$ \\
\hline MVS-135-XRF & 2.158 & & 87 & & 66 & & 249 & & 2.0 & & $\mathrm{~J}$ & 0.7 & & $\mathrm{U}$ \\
\hline MVS-136-XRF & 1.975 & & 75 & & 167 & & 191 & & 0.1 & & $\mathrm{U}$ & 0.1 & & $\mathrm{U}$ \\
\hline MVS-137-XRF & 2.177 & & 79 & & 50 & & 117 & & 1.2 & & $\mathrm{~J}$ & 0.1 & & U \\
\hline MVS-138-XRF & 2.429 & & 146 & & 107 & & 250 & & 0.3 & & $\mathrm{U}$ & 0.9 & & $\mathrm{U}$ \\
\hline MVS-139-XRF & 2.337 & 0.029 & 78 & 4 & 49 & 5 & 102 & 4 & 1.5 & 0.9 & $\mathrm{~J}$ & 0.9 & 0.5 & $\mathrm{U}$ \\
\hline MVS-140-XRF & 2.318 & & 108 & & 49 & & 120 & & 2.1 & & $\mathrm{~J}$ & 1.6 & & $\mathrm{~J}$ \\
\hline MVS-141-XRF & 2.282 & & 149 & & 113 & & 279 & & 1.5 & & $\mathrm{~J}$ & ND & & $\mathrm{U}$ \\
\hline MVS-142-XRF & 2.091 & & 126 & & 75 & & 291 & & 1.1 & & $\mathrm{~J}$ & 1.2 & & $\mathrm{~J}$ \\
\hline MVS-143-XRF & 2.348 & & 87 & & 41 & & 105 & & 0.6 & & $\mathrm{U}$ & 0.1 & & U \\
\hline MVS-144-XRF & 2.359 & 0.011 & 88 & 4 & 51 & 2 & 114 & 4 & 0.9 & 0.3 & $\mathrm{U}$ & 1.2 & 0.4 & $\mathrm{~J}$ \\
\hline MVS-145-XRF & 2.405 & & 211 & & 140 & & 283 & & 1.9 & & $\mathrm{~J}$ & 0.4 & & $\mathrm{U}$ \\
\hline MVS-146-XRF & 2.413 & & 66 & & 39 & & 92 & & 0.7 & & $\mathrm{U}$ & 0.8 & & U \\
\hline MVS-147-XRF & 2.160 & & 67 & & 48 & & 109 & & 1.4 & & $\mathrm{~J}$ & 0.2 & & U \\
\hline MVS-148-XRF & 2.158 & & 99 & & 52 & & 137 & & 2.7 & & $\mathrm{~J}$ & 1.4 & & $\mathrm{~J}$ \\
\hline MVS-149-XRF & 2.067 & 0.020 & 71 & 3 & 41 & 5 & 104 & 10 & 1.1 & 1.1 & $\mathrm{~J}$ & 0.3 & 0.3 & $\mathrm{U}$ \\
\hline MVS-150-XRF & 1.863 & & 49 & & 27 & & 61 & & ND & & $\mathrm{U}$ & ND & & U \\
\hline MVS-151-XRF & 2.394 & & 181 & & 94 & & 425 & & 0.7 & & $\mathrm{U}$ & 1.3 & & $\mathrm{~J}$ \\
\hline MVS-152-XRF & 2.155 & & 88 & & 44 & & 99 & & 0.8 & & $\mathrm{U}$ & 0.6 & & $\mathrm{U}$ \\
\hline MVS-153-XRF & 2.394 & & 98 & & 55 & & 132 & & ND & & $\mathrm{U}$ & 0.6 & & U \\
\hline MVS-154-XRF & 1.993 & & 79 & & 39 & & 96 & & 2.6 & & $\mathrm{~J}$ & 1.0 & & $\mathrm{~J}$ \\
\hline MVS-155-XRF & 2.362 & & 89 & & 47 & & 125 & & 0.8 & & $\mathrm{U}$ & 0.6 & & $\mathrm{U}$ \\
\hline MVS-156-XRF & 2.502 & & 49 & & 29 & & 81 & & 2.2 & & $\mathrm{~J}$ & 0.6 & & $\mathrm{U}$ \\
\hline MVS-157-XRF & 2.423 & & 68 & & 36 & & 97 & & 0.1 & & $\mathrm{U}$ & 1.4 & & $\mathrm{~J}$ \\
\hline MVS-158-XRF & 2.192 & & 57 & & 31 & & 76 & & ND & & $\mathrm{U}$ & 0.1 & & $\mathrm{U}$ \\
\hline MVS-159-XRF & 2.193 & & 114 & & 60 & & 151 & & 2.2 & & $\mathrm{~J}$ & 1.5 & & $\mathrm{~J}$ \\
\hline MVS-160-XRF & 2.018 & & 78 & & 39 & & 100 & & 1.4 & & $\mathrm{~J}$ & 1.3 & & $\mathrm{~J}$ \\
\hline MVS-161-XRF & 2.210 & & 80 & & 43 & & 93 & & 0.3 & & $\mathrm{U}$ & 0.1 & & U \\
\hline MVS-162-XRF & 1.889 & & 76 & & 39 & & 114 & & 1.3 & & $\mathrm{~J}$ & ND & & $\mathrm{U}$ \\
\hline MVS-163-XRF & 2.149 & & 79 & & 44 & & 101 & & 0.5 & & $\mathrm{U}$ & 0.1 & & U \\
\hline MVS-164-XRF & 2.345 & & 46 & & 25 & & 76 & & 1.6 & & $\mathrm{~J}$ & 0.1 & & U \\
\hline
\end{tabular}


Project:

Samples Received:

Metals Reported:
ENVVEST Metals Verification Study (Project No. 43043)

9/4/2003

Fe, Cu, Pb, Zn, Cd, Ag

Rapid Screening Method: EDXRF (wet)

(concentrations in mg/kg, except where noted; not blank corrected)

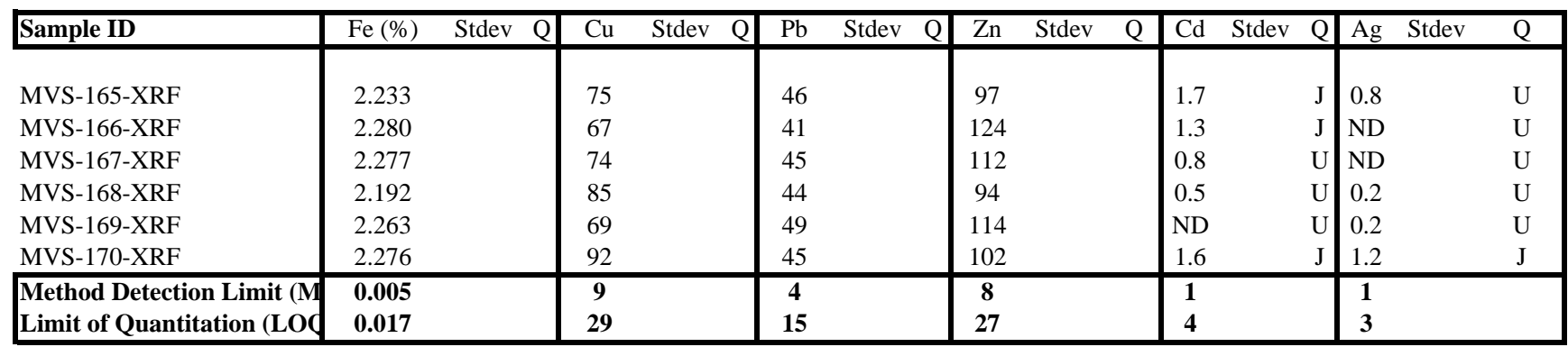

Data Qualifiers (Q):

U: The value was less than MDL or the analyte was not detected.

J: Estimated value ( MDL < measured value $<$ LOQ ) 
Project: $\quad$ ENVVEST Metals Verification Study (Project No. 43043)

Samples Received: 9/4/2003 (Dyes Inlet, Ostrich Bay, Port Orchard Passage, Rich Passage)

Metals Reported: $\quad$ Fe, Cu, Pb, Zn, Cd, Ag

\section{Data Quality Criteria}

1. Accuracy

SRMs

PACS-1

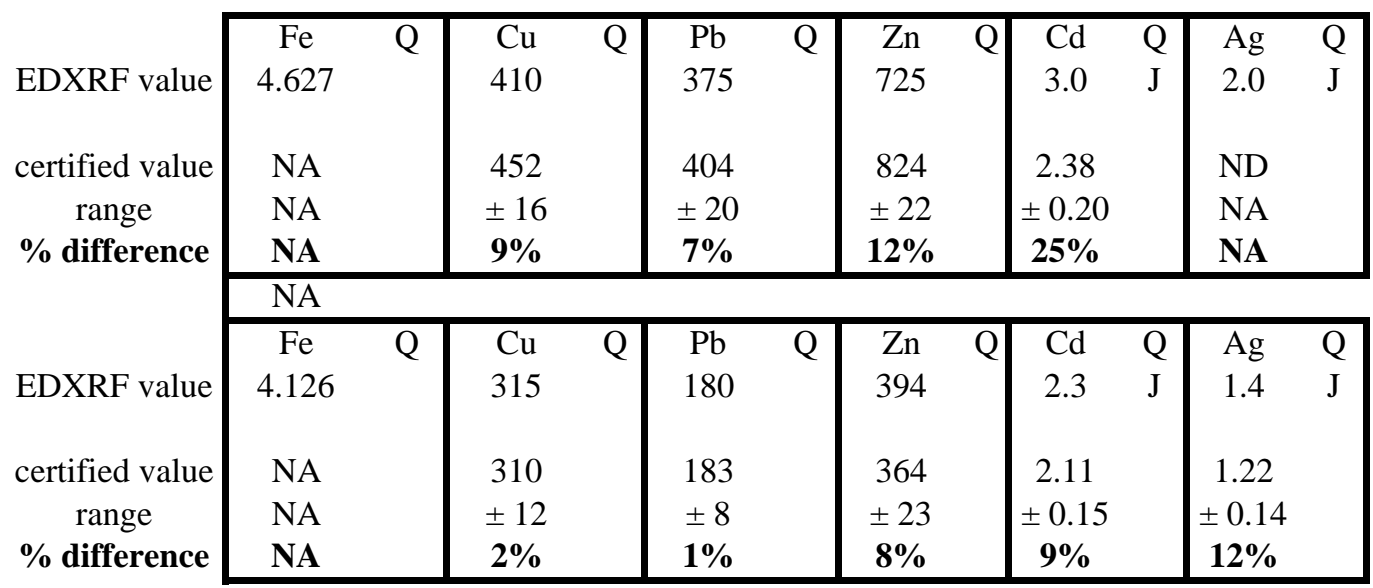

2. Precision

SAMPLE TRIPLICATE (for samples triplicates only, instrument precision)

Sample ID

MVS-003

MVS-003

MVS-003

\begin{tabular}{cc|cc|cc|cc|cc|cc|cc|}
\cline { 3 - 10 } & & $\mathrm{Fe}$ & $\mathrm{Q}$ & $\mathrm{Cu}$ & $\mathrm{Q}$ & $\mathrm{Pb}$ & $\mathrm{Q}$ & $\mathrm{Zn}$ & $\mathrm{Q}$ & $\mathrm{Cd}$ & $\mathrm{Q}$ & $\mathrm{Ag}$ & $\mathrm{Q}$ \\
$\mathrm{A}$ & MVS-003-A & 2.045 & & 42 & & 25 & & 83 & & 0.6 & $\mathrm{U}$ & 0.0 & $\mathrm{U}$ \\
$\mathrm{B}$ & MVS-003-B & 2.074 & & 46 & & 33 & & 80 & & 0.4 & $\mathrm{U}$ & 0.3 & $\mathrm{U}$ \\
$\mathrm{C}$ & MVS-003-C & 2.093 & & 49 & & 31 & & 88 & & 0.4 & $\mathrm{U}$ & 0.6 & $\mathrm{U}$ \\
& mean & 2.071 & & 46 & & 30 & & 84 & & 0.5 & $\mathrm{U}$ & 0.3 & $\mathrm{U}$ \\
& stdev & 0.024 & & 3 & & 4 & & 4 & & 0.1 & & 0.3 & \\
& \%RSD & $\mathbf{1}$ & $\mathbf{8}$ & & $\mathbf{1 4}$ & & $\mathbf{5}$ & & $\mathbf{2 7}$ & & $\mathbf{9 8}$ & \\
\hline
\end{tabular}

Sample ID

MVS-007

MVS-007

MVS-007

\begin{tabular}{cc|cc|cc|cc|cc|cc|cc|} 
& & Fe & Q & Cu & Q & Pb & Q & Zn & Q & Cd & Q & Ag & Q \\
A & MVS-007-A & 2.170 & & 59 & & 41 & & 98 & & 0.0 & U & 0.8 & U \\
B & MVS-007-B & 2.240 & & 61 & & 36 & & 97 & & 0.4 & U & 1.4 & J \\
C & MVS-007-C & 2.215 & & 51 & & 41 & & 97 & & 1.2 & J & 0.1 & U \\
& mean & 2.208 & & 57 & & 39 & & 97 & & 0.6 & U & 0.7 & U \\
& stdev & 0.035 & & 5 & & 3 & & 1 & & 0.6 & & 0.6 & \\
& \%RSD & $\mathbf{2}$ & & $\mathbf{9}$ & & $\mathbf{8}$ & & $\mathbf{1}$ & & $\mathbf{1 1 4}$ & & $\mathbf{8 5}$ & \\
\hline
\end{tabular}


Project: $\quad$ ENVVEST Metals Verification Study (Project No. 43043)

Samples Received: 9/4/2003 (Dyes Inlet, Ostrich Bay, Port Orchard Passage, Rich Passage)

Metals Reported: $\quad$ Fe, Cu, Pb, Zn, Cd, Ag

\section{Data Quality Criteria}

SAMPLE TRIPLICATE (cont'd)

Sample ID

MVS-010

MVS-010

MVS-010

MVS-015

MVS-015

MVS-015

Sample ID

MVS-016

MVS-016

MVS-016

A MVS-016-A

B MVS-016-B

C MVS-016-C

mean

stdev

\%RSD

\begin{tabular}{|cc|cc|cc|cc|cc|cc|}
\hline $\mathrm{Fe}$ & $\mathrm{Q}$ & $\mathrm{Cu}$ & $\mathrm{Q}$ & $\mathrm{Pb}$ & $\mathrm{Q}$ & $\mathrm{Zn}$ & $\mathrm{Q}$ & $\mathrm{Cd}$ & $\mathrm{Q}$ & $\mathrm{Ag}$ & $\mathrm{Q}$ \\
2.059 & & 47 & & 29 & & 88 & & 0.2 & $\mathrm{U}$ & 0.0 & $\mathrm{U}$ \\
2.075 & & 48 & & 35 & & 95 & & 0.9 & $\mathrm{U}$ & 0.0 & $\mathrm{U}$ \\
2.086 & & 47 & & 28 & & 90 & & 0.5 & $\mathrm{U}$ & 0.6 & $\mathrm{U}$ \\
2.073 & & 47 & & 31 & & 91 & & 0.5 & $\mathrm{U}$ & 0.2 & $\mathrm{U}$ \\
0.014 & & 1 & & 4 & & 3 & & 0.3 & & 0.3 & \\
$\mathbf{1}$ & $\mathbf{2}$ & & $\mathbf{1 2}$ & & $\mathbf{4}$ & $\mathbf{6 1}$ & & $\mathbf{1 7 3}$ & \\
\hline
\end{tabular}

\begin{tabular}{|cc|cc|cc|cc|cc|cc|}
\hline $\mathrm{Fe}$ & $\mathrm{Q}$ & $\mathrm{Cu}$ & $\mathrm{Q}$ & $\mathrm{Pb}$ & $\mathrm{Q}$ & $\mathrm{Zn}$ & $\mathrm{Q}$ & $\mathrm{Cd}$ & $\mathrm{Q}$ & $\mathrm{Ag}$ & $\mathrm{Q}$ \\
3.000 & & 30 & & 10 & $\mathrm{~J}$ & 63 & & 0.0 & $\mathrm{U}$ & 0.7 & $\mathrm{U}$ \\
3.105 & & 30 & & 10 & $\mathrm{~J}$ & 58 & & 0.0 & $\mathrm{U}$ & 0.0 & $\mathrm{U}$ \\
2.992 & & 24 & $\mathrm{~J}$ & 8 & $\mathrm{~J}$ & 55 & & 0.0 & $\mathrm{U}$ & 0.5 & $\mathrm{U}$ \\
3.032 & & 28 & $\mathrm{~J}$ & 9 & $\mathrm{~J}$ & 59 & & 0.0 & $\mathrm{U}$ & 0.4 & $\mathrm{U}$ \\
0.063 & & 3 & & 1 & & 4 & & 0.0 & & 0.4 & \\
$\mathbf{2}$ & & $\mathbf{1 1}$ & & $\mathbf{1 1}$ & & $\mathbf{7}$ & $\mathbf{N A}$ & & $\mathbf{8 9}$ & \\
\hline
\end{tabular}

\begin{tabular}{|cc|cc|cc|cc|cc|cc|}
\hline $\mathrm{Fe}$ & $\mathrm{Q}$ & $\mathrm{Cu}$ & $\mathrm{Q}$ & $\mathrm{Pb}$ & $\mathrm{Q}$ & $\mathrm{Zn}$ & $\mathrm{Q}$ & $\mathrm{Cd}$ & $\mathrm{Q}$ & $\mathrm{Ag}$ & $\mathrm{Q}$ \\
2.442 & & 39 & & 14 & $\mathrm{~J}$ & 64 & & 1.1 & $\mathrm{~J}$ & 0.0 & $\mathrm{U}$ \\
2.446 & & 31 & & 13 & $\mathrm{~J}$ & 64 & & 0.7 & $\mathrm{U}$ & 0.0 & $\mathrm{U}$ \\
2.479 & & 32 & & 14 & $\mathrm{~J}$ & 65 & & 0.3 & $\mathrm{U}$ & 0.2 & $\mathrm{U}$ \\
2.456 & & 34 & & 14 & $\mathrm{~J}$ & 65 & & 0.7 & $\mathrm{U}$ & 0.1 & $\mathrm{U}$ \\
0.020 & & 4 & & 1 & & 0 & & 0.4 & & 0.1 & \\
$\mathbf{1}$ & $\mathbf{1 2}$ & & $\mathbf{4}$ & & $\mathbf{1}$ & $\mathbf{5 5}$ & & $\mathbf{1 5 0}$ & \\
\hline
\end{tabular}

Sample ID

MVS-021

MVS-021

MVS-021

$$
\begin{array}{cc}
\text { A } & \text { MVS-021-A } \\
\text { B } & \text { MVS-021-B } \\
\text { C } & \text { MVS-021-C } \\
& \text { mean } \\
& \text { stdev } \\
& \text { \%RSD }
\end{array}
$$

\begin{tabular}{|cc|cc|cc|cc|cc|cc|}
\hline $\mathrm{Fe}$ & $\mathrm{Q}$ & $\mathrm{Cu}$ & $\mathrm{Q}$ & $\mathrm{Pb}$ & $\mathrm{Q}$ & $\mathrm{Zn}$ & $\mathrm{Q}$ & $\mathrm{Cd}$ & $\mathrm{Q}$ & $\mathrm{Ag}$ & $\mathrm{Q}$ \\
1.970 & & 49 & & 23 & & 79 & & 0.7 & $\mathrm{U}$ & 1.6 & $\mathrm{~J}$ \\
2.044 & & 41 & & 27 & & 72 & & 0.0 & $\mathrm{U}$ & 1.2 & $\mathrm{~J}$ \\
1.991 & & 48 & & 29 & & 65 & & 2.3 & $\mathrm{~J}$ & 1.2 & $\mathrm{~J}$ \\
2.002 & & 46 & & 26 & & 72 & & 1.0 & $\mathrm{~J}$ & 1.3 & $\mathrm{~J}$ \\
0.038 & & 4 & & 3 & & 7 & & 1.2 & & 0.2 & \\
$\mathbf{2}$ & & $\mathbf{9}$ & & $\mathbf{1 1}$ & & $\mathbf{1 0}$ & & $\mathbf{1 1 9}$ & & $\mathbf{1 8}$ & \\
\hline
\end{tabular}

Sample ID

MVS-025

MVS-025

MVS-025

A MVS-025-A

B MVS-025-B

C MVS-025-C

mean

stdev

\%RSD

\begin{tabular}{|cc|cc|cc|cc|cc|cc|}
\hline $\mathrm{Fe}$ & $\mathrm{Q}$ & $\mathrm{Cu}$ & $\mathrm{Q}$ & $\mathrm{Pb}$ & $\mathrm{Q}$ & $\mathrm{Zn}$ & $\mathrm{Q}$ & $\mathrm{Cd}$ & $\mathrm{Q}$ & $\mathrm{Ag}$ & $\mathrm{Q}$ \\
2.009 & & 52 & & 30 & & 66 & & 0.0 & $\mathrm{U}$ & 0.1 & $\mathrm{U}$ \\
1.968 & & 38 & & 24 & & 77 & & 0.9 & $\mathrm{U}$ & 0.4 & $\mathrm{U}$ \\
1.980 & & 41 & & 30 & & 71 & & 1.1 & $\mathrm{~J}$ & 0.9 & $\mathrm{U}$ \\
1.986 & & 44 & & 28 & & 71 & & 0.7 & $\mathrm{U}$ & 0.5 & $\mathrm{U}$ \\
0.021 & & 8 & & 3 & & 5 & & 0.6 & & 0.4 & \\
$\mathbf{1}$ & & $\mathbf{1 7}$ & & $\mathbf{1 2}$ & & $\mathbf{7}$ & $\mathbf{8 7}$ & & $\mathbf{9 5}$ & \\
\hline
\end{tabular}


Project: $\quad$ ENVVEST Metals Verification Study (Project No. 43043)

Samples Received: 9/4/2003 (Dyes Inlet, Ostrich Bay, Port Orchard Passage, Rich Passage)

Metals Reported: $\quad$ Fe, Cu, Pb, Zn, Cd, Ag

\section{Data Quality Criteria}

SAMPLE TRIPLICATE (cont'd)

Sample ID

MVS-027

MVS-027

MVS-027

\begin{tabular}{cc|cc|cc|cc|cc|cc|cc|}
\cline { 3 - 11 } & & $\mathrm{Fe}$ & $\mathrm{Q}$ & $\mathrm{Cu}$ & $\mathrm{Q}$ & $\mathrm{Pb}$ & $\mathrm{Q}$ & $\mathrm{Zn}$ & $\mathrm{Q}$ & $\mathrm{Cd}$ & $\mathrm{Q}$ & $\mathrm{Ag}$ & $\mathrm{Q}$ \\
$\mathrm{A}$ & MVS-027-A & 2.090 & & 55 & & 25 & & 80 & & 1.3 & $\mathrm{~J}$ & 0.0 & $\mathrm{U}$ \\
$\mathrm{B}$ & MVS-027-B & 2.072 & & 51 & & 35 & & 69 & & 0.3 & $\mathrm{U}$ & 0.0 & $\mathrm{U}$ \\
$\mathrm{C}$ & MVS-027-C & 2.086 & & 45 & & 30 & & 81 & & 0.8 & $\mathrm{U}$ & 0.9 & $\mathrm{U}$ \\
& mean & 2.083 & & 50 & & 30 & & 77 & & 0.8 & $\mathrm{U}$ & 0.3 & $\mathrm{U}$ \\
& stdev & 0.009 & & 5 & & 5 & & 7 & & 0.5 & & 0.5 & \\
& \%RSD & $\mathbf{0}$ & & $\mathbf{1 1}$ & & $\mathbf{1 8}$ & & $\mathbf{9}$ & & $\mathbf{6 5}$ & & $\mathbf{1 6 8}$ & \\
\hline
\end{tabular}

Sample ID

MVS-042

MVS-042

MVS-042

\begin{tabular}{cc|cc|cc|cc|cc|cc|cc|}
\cline { 3 - 10 } & & $\mathrm{Fe}$ & $\mathrm{Q}$ & $\mathrm{Cu}$ & $\mathrm{Q}$ & $\mathrm{Pb}$ & $\mathrm{Q}$ & $\mathrm{Zn}$ & $\mathrm{Q}$ & $\mathrm{Cd}$ & $\mathrm{Q}$ & $\mathrm{Ag}$ & $\mathrm{Q}$ \\
$\mathrm{A}$ & MVS-042-A & 1.991 & & 25 & $\mathrm{~J}$ & 15 & & 53 & & 1.9 & $\mathrm{~J}$ & 0.4 & $\mathrm{U}$ \\
$\mathrm{B}$ & MVS-042-B & 1.985 & & 28 & $\mathrm{~J}$ & 16 & & 53 & & 0.1 & $\mathrm{U}$ & 0.6 & $\mathrm{U}$ \\
$\mathrm{C}$ & MVS-042-C & 1.921 & & 24 & $\mathrm{~J}$ & 24 & & 50 & & 0.0 & $\mathrm{U}$ & 0.0 & $\mathrm{U}$ \\
& mean & 1.966 & & 25 & $\mathrm{~J}$ & 18 & & 52 & & 0.7 & $\mathrm{U}$ & 0.3 & $\mathrm{U}$ \\
& stdev & 0.039 & & 2 & & 5 & & 2 & & 1.1 & & 0.3 & \\
& \%RSD & $\mathbf{2}$ & $\mathbf{7}$ & & $\mathbf{2 7}$ & & $\mathbf{3}$ & $\mathbf{1 5 9}$ & & $\mathbf{9 2}$ & \\
\hline
\end{tabular}

Sample ID

MVS-045

MVS-045

MVS-045

\begin{tabular}{cc|cc|cc|cc|cc|cc|cc|}
\cline { 3 - 9 } & & $\mathrm{Fe}$ & $\mathrm{Q}$ & $\mathrm{Cu}$ & $\mathrm{Q}$ & $\mathrm{Pb}$ & $\mathrm{Q}$ & $\mathrm{Zn}$ & $\mathrm{Q}$ & $\mathrm{Cd}$ & $\mathrm{Q}$ & $\mathrm{Ag}$ & $\mathrm{Q}$ \\
$\mathrm{A}$ & MVS-045-A & 2.065 & & 34 & & 23 & & 64 & & 0.5 & $\mathrm{U}$ & 0.0 & $\mathrm{U}$ \\
$\mathrm{B}$ & MVS-045-B & 2.026 & & 41 & & 23 & & 59 & & 1.0 & $\mathrm{~J}$ & 0.0 & $\mathrm{U}$ \\
$\mathrm{C}$ & MVS-045-C & 1.991 & & 29 & & 22 & & 61 & & 0.1 & $\mathrm{U}$ & 0.6 & $\mathrm{U}$ \\
& mean & 2.027 & & 35 & & 23 & & 62 & & 0.5 & $\mathrm{U}$ & 0.2 & $\mathrm{U}$ \\
& stdev & 0.037 & & 6 & & 1 & & 2 & & 0.5 & & 0.4 & \\
& \%RSD & $\mathbf{2}$ & $\mathbf{1 8}$ & $\mathbf{4}$ & & $\mathbf{4}$ & & $\mathbf{8 5}$ & & $\mathbf{1 7 3}$ & \\
\hline
\end{tabular}

Sample ID

MVS-052

MVS-052

A MVS-052-A

\begin{tabular}{|cc|cc|cc|cc|cc|cc|}
\hline $\mathrm{Fe}$ & $\mathrm{Q}$ & $\mathrm{Cu}$ & $\mathrm{Q}$ & $\mathrm{Pb}$ & $\mathrm{Q}$ & $\mathrm{Zn}$ & $\mathrm{Q}$ & $\mathrm{Cd}$ & $\mathrm{Q}$ & $\mathrm{Ag}$ & $\mathrm{Q}$ \\
2.059 & & 37 & & 27 & & 80 & & 2.6 & $\mathrm{~J}$ & 0.5 & $\mathrm{U}$ \\
2.026 & & 37 & & 30 & & 75 & & 1.8 & $\mathrm{~J}$ & 0.2 & $\mathrm{U}$ \\
2.064 & & 37 & & 27 & & 79 & & 2.7 & $\mathrm{~J}$ & 0.4 & $\mathrm{U}$ \\
2.050 & & 37 & & 28 & & 78 & & 2.3 & $\mathrm{~J}$ & 0.4 & $\mathrm{U}$ \\
0.021 & & 0 & & 2 & & 3 & & 0.5 & & 0.2 & \\
$\mathbf{1}$ & & $\mathbf{0}$ & & $\mathbf{6}$ & & $\mathbf{3}$ & & $\mathbf{2 1}$ & & $\mathbf{4 8}$ & \\
\hline
\end{tabular}

MVS-052

B MVS-052-B

C MVS-052-C

mean

stdev

\%RSD

$$
1
$$

\section{Data Qualifiers (Q):}

U: The value was less than MDL or the analyte was not detected.

J: Estimated value ( MDL < measured value $<$ LOQ ) 
Project: $\quad$ ENVVEST Metals Verification Study (Project No. 43043)

Samples Received: 11/20/2003 (Sinclair Inlet OU B Marine monitoring composites)

Metals Reported: $\quad$ Fe, Cu, Pb, Zn, Cd, Ag

\section{Data Quality Criteria}

1. Accuracy

SRMs

PACS-1

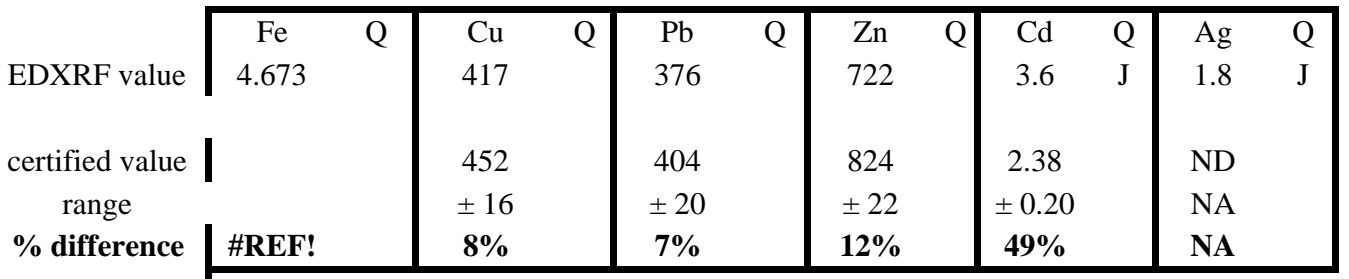

PACS-2

EDXRF value

\begin{tabular}{|cc|c}
\hline NA & \\
\hline Fe & Q & C \\
4.182 & & 312 \\
& & \\
NA & & 310 \\
NA & & \pm \\
NA & & $\mathbf{1}$ \\
\hline
\end{tabular}

PACS-2

certified value range $\%$ difference

NA

\begin{tabular}{cc|c}
$\mathrm{Cu}$ & $\mathrm{Q}$ & $\mathrm{Pb}$ \\
312 & & 180 \\
& & \\
310 & & 18 \\
\pm 12 & & \pm \\
$\mathbf{1 \%}$ & & $\mathbf{2 \%}$ \\
\hline
\end{tabular}

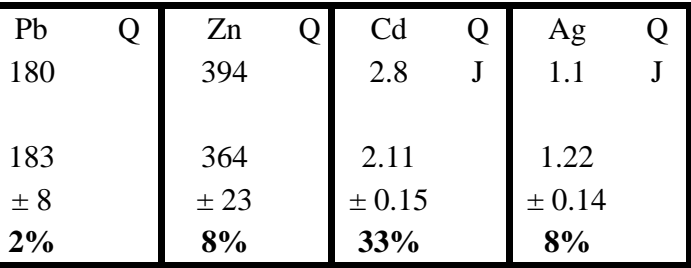

2. Precision

SAMPLE TRIPLICATE (for samples triplicates only, instrument precision)

Sample ID

MVS-063

MVS-063

MVS-063

B MVS-063-B

C MVS-063-C

mean

stdev

\%RSD

\begin{tabular}{|cc|cc|cc|cc|cc|cc|}
\hline $\mathrm{Fe}$ & $\mathrm{Q}$ & $\mathrm{Cu}$ & $\mathrm{Q}$ & $\mathrm{Pb}$ & $\mathrm{Q}$ & $\mathrm{Zn}$ & $\mathrm{Q}$ & $\mathrm{Cd}$ & $\mathrm{Q}$ & $\mathrm{Ag}$ & $\mathrm{Q}$ \\
2.272 & & 59 & & 43 & & 97 & & 0.0 & $\mathrm{U}$ & 0.2 & $\mathrm{U}$ \\
2.282 & & 60 & & 34 & & 92 & & 0.8 & $\mathrm{U}$ & 1.6 & $\mathrm{~J}$ \\
2.244 & & 66 & & 41 & & 100 & & 0.5 & $\mathrm{U}$ & 0.8 & $\mathrm{U}$ \\
2.266 & & 62 & & 39 & & 96 & 0.4 & $\mathrm{U}$ & 0.9 & $\mathrm{U}$ \\
0.020 & 3 & & 5 & & 4 & 0.4 & & 0.7 & \\
$\mathbf{1}$ & & $\mathbf{6}$ & & $\mathbf{1 2}$ & & $\mathbf{4}$ & $\mathbf{9 3}$ & & $\mathbf{8 7}$ & \\
\hline
\end{tabular}

Sample ID

MVS-072

MVS-072

MVS-072

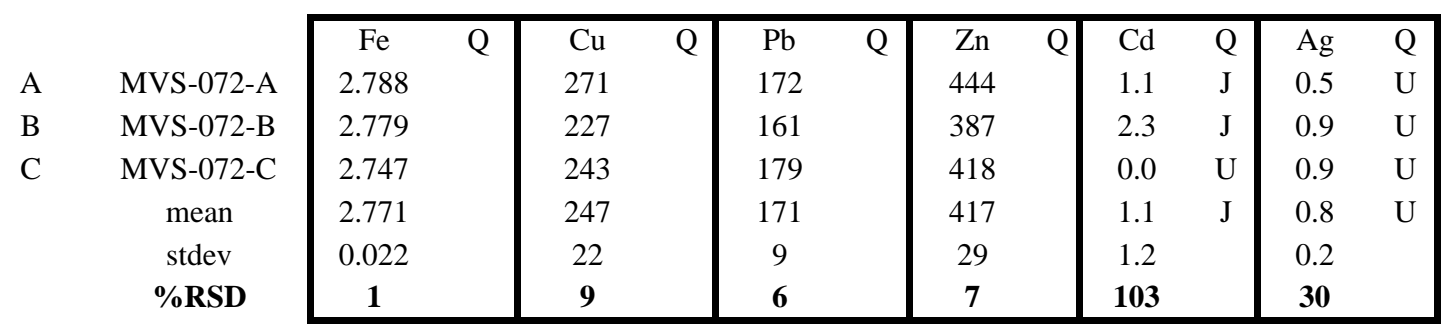


Project: $\quad$ ENVVEST Metals Verification Study (Project No. 43043)

Samples Received: 11/20/2003 (Sinclair Inlet OU B Marine monitoring composites)

Metals Reported: $\quad \mathrm{Fe}, \mathrm{Cu}, \mathrm{Pb}, \mathrm{Zn}, \mathrm{Cd}, \mathrm{Ag}$

\section{Data Quality Criteria}

SAMPLE TRIPLICATE (cont'd)

Sample ID

MVS-080

MVS-080
MVS-080

$$
\begin{array}{cc}
\text { A } & \text { MVS-080-A } \\
\text { B } & \text { MVS-080-B } \\
\text { C } & \text { MVS-080-C } \\
& \text { mean } \\
& \text { stdev } \\
& \text { \%RSD }
\end{array}
$$

\begin{tabular}{|cc|cc|cc|cc|cc|cc|}
\hline $\mathrm{Fe}$ & $\mathrm{Q}$ & $\mathrm{Cu}$ & $\mathrm{Q}$ & $\mathrm{Pb}$ & $\mathrm{Q}$ & $\mathrm{Zn}$ & $\mathrm{Q}$ & $\mathrm{Cd}$ & $\mathrm{Q}$ & $\mathrm{Ag}$ & $\mathrm{Q}$ \\
2.185 & & 44 & & 32 & & 79 & & 1.2 & $\mathrm{~J}$ & 0.9 & $\mathrm{U}$ \\
2.159 & & 47 & & 25 & & 81 & & 0.0 & $\mathrm{U}$ & 0.0 & $\mathrm{U}$ \\
2.145 & & 38 & & 28 & & 77 & & 0.1 & $\mathrm{U}$ & 0.5 & $\mathrm{U}$ \\
2.163 & & 43 & & 28 & & 79 & & 0.4 & $\mathrm{U}$ & 0.5 & $\mathrm{U}$ \\
0.020 & 5 & & 3 & & 2 & & 0.6 & & 0.5 & \\
$\mathbf{1}$ & & $\mathbf{1 1}$ & & $\mathbf{1 2}$ & & $\mathbf{3}$ & & $\mathbf{1 4 6}$ & & $\mathbf{1 0 0}$ & \\
\hline
\end{tabular}

Sample ID MVS-085

MVS-085

MVS-085

Sample ID MVS-094

MVS-094

MVS-094

$$
\begin{array}{cc}
\text { A } & \text { MVS-094-A } \\
\text { B } & \text { MVS-094-B } \\
\text { C } & \text { MVS-094-C } \\
& \text { mean } \\
& \text { stdev }
\end{array}
$$

\%RSD

Sample ID

MVS-101

MVS-101

MVS-101

$$
\begin{array}{cc}
\text { A } & \text { MVS-101-A } \\
\text { B } & \text { MVS-101-B } \\
\text { C } & \text { MVS-101-C } \\
& \text { mean } \\
& \text { stdev }
\end{array}
$$$$
\text { \%RSD }
$$

\begin{tabular}{|c|c|c|}
\hline $\mathrm{Fe}$ & $\mathrm{Q}$ & $\mathrm{C}$ \\
2.233 & & 5 \\
2.239 & & 63 \\
2.275 & & 79 \\
2.249 & & 66 \\
0.023 & & 12 \\
$\mathbf{1}$ & $\mathbf{1}$ \\
\hline
\end{tabular}

\begin{tabular}{|ll|cc|cc|cc|cc|}
$\mathrm{Cu}$ & $\mathrm{Q}$ & $\mathrm{Pb}$ & $\mathrm{Q}$ & $\mathrm{Zn}$ & $\mathrm{Q}$ & $\mathrm{Cd}$ & $\mathrm{Q}$ & $\mathrm{Ag}$ & $\mathrm{Q}$ \\
56 & & 36 & & 98 & & 0.0 & $\mathrm{U}$ & 1.4 & $\mathrm{~J}$ \\
63 & & 35 & & 99 & & 0.0 & $\mathrm{U}$ & 1.0 & $\mathrm{~J}$ \\
79 & & 43 & & 100 & & 0.9 & $\mathrm{U}$ & 2.1 & $\mathrm{~J}$ \\
66 & 38 & & 99 & & 0.3 & $\mathrm{U}$ & 1.5 & $\mathrm{~J}$ \\
12 & & 4 & & 1 & & 0.5 & & 0.6 & \\
$\mathbf{1 8}$ & & $\mathbf{1 2}$ & & $\mathbf{1}$ & & $\mathbf{1 5 9}$ & & $\mathbf{3 9}$ & \\
\hline
\end{tabular}

\begin{tabular}{|cc|cc|cc|cc|cc|cc|}
\hline $\mathrm{Fe}$ & $\mathrm{Q}$ & $\mathrm{Cu}$ & $\mathrm{Q}$ & $\mathrm{Pb}$ & $\mathrm{Q}$ & $\mathrm{Zn}$ & $\mathrm{Q}$ & $\mathrm{Cd}$ & $\mathrm{Q}$ & $\mathrm{Ag}$ & $\mathrm{Q}$ \\
2.388 & & 180 & & 85 & & 243 & & 1.5 & $\mathrm{~J}$ & 1.1 & $\mathrm{~J}$ \\
2.359 & & 143 & & 114 & & 267 & & 1.7 & $\mathrm{~J}$ & 0.5 & $\mathrm{U}$ \\
2.370 & & 131 & & 94 & & 232 & & 2.6 & $\mathrm{~J}$ & 1.2 & $\mathrm{~J}$ \\
2.372 & & 152 & & 98 & & 247 & & 2.0 & $\mathrm{~J}$ & 1.0 & $\mathrm{~J}$ \\
0.015 & & 25 & & 15 & & 18 & 0.6 & & 0.4 & \\
$\mathbf{1}$ & $\mathbf{1 7}$ & & $\mathbf{1 5}$ & & $\mathbf{7}$ & $\mathbf{3 1}$ & & $\mathbf{3 8}$ & \\
\hline
\end{tabular}

Sample ID MVS-107 MVS-107 MVS-107

\begin{tabular}{|cc|cc|cc|cc|cc|cc|}
\hline $\mathrm{Fe}$ & $\mathrm{Q}$ & $\mathrm{Cu}$ & $\mathrm{Q}$ & $\mathrm{Pb}$ & $\mathrm{Q}$ & $\mathrm{Zn}$ & $\mathrm{Q}$ & $\mathrm{Cd}$ & $\mathrm{Q}$ & $\mathrm{Ag}$ & $\mathrm{Q}$ \\
2.313 & & 74 & & 42 & & 104 & & 0.0 & $\mathrm{U}$ & 1.6 & $\mathrm{~J}$ \\
2.323 & & 87 & & 46 & & 102 & & 2.2 & $\mathrm{~J}$ & 0.0 & $\mathrm{U}$ \\
2.339 & & 89 & & 46 & & 96 & 0.8 & $\mathrm{U}$ & 0.4 & $\mathrm{U}$ \\
2.325 & & 83 & & 45 & & 101 & 1.0 & $\mathrm{~J}$ & 0.7 & $\mathrm{U}$ \\
0.013 & & 8 & & 2 & & 4 & 1.1 & & 0.8 & \\
$\mathbf{1}$ & & $\mathbf{1 0}$ & & $\mathbf{5}$ & & $\mathbf{4}$ & $\mathbf{1 1 3}$ & & $\mathbf{1 2 5}$ & \\
\hline
\end{tabular}

$$
\begin{array}{cc}
\text { A } & \text { MVS-107-A } \\
\text { B } & \text { MVS-107-B } \\
\text { C } & \text { MVS-107-C } \\
& \text { mean } \\
& \text { stdev }
\end{array}
$$$$
\text { \%RSD }
$$

\begin{tabular}{|cc|cc|cc|cc|cc|cc|}
\hline $\mathrm{Fe}$ & $\mathrm{Q}$ & $\mathrm{Cu}$ & $\mathrm{Q}$ & $\mathrm{Pb}$ & $\mathrm{Q}$ & $\mathrm{Zn}$ & $\mathrm{Q}$ & $\mathrm{Cd}$ & $\mathrm{Q}$ & $\mathrm{Ag}$ & $\mathrm{Q}$ \\
2.189 & & 95 & & 47 & & 107 & & 1.9 & $\mathrm{~J}$ & 2.2 & $\mathrm{~J}$ \\
2.247 & & 91 & & 42 & & 116 & & 0.9 & $\mathrm{U}$ & 1.8 & $\mathrm{~J}$ \\
2.184 & & 90 & & 45 & & 105 & & 3.0 & $\mathrm{~J}$ & 1.1 & $\mathrm{~J}$ \\
2.207 & & 92 & & 45 & & 110 & 1.9 & $\mathrm{~J}$ & 1.7 & $\mathrm{~J}$ \\
0.035 & & 2 & & 3 & & 6 & 1.1 & & 0.6 & \\
$\mathbf{2}$ & & $\mathbf{3}$ & & $\mathbf{6}$ & & $\mathbf{5}$ & $\mathbf{5 7}$ & & $\mathbf{3 4}$ & \\
\hline
\end{tabular}


Project: $\quad$ ENVVEST Metals Verification Study (Project No. 43043)

Samples Received: 11/20/2003 (Sinclair Inlet OU B Marine monitoring composites)

Metals Reported: $\quad \mathrm{Fe}, \mathrm{Cu}, \mathrm{Pb}, \mathrm{Zn}, \mathrm{Cd}, \mathrm{Ag}$

\section{Data Quality Criteria}

SAMPLE TRIPLICATE (cont'd)

Sample ID

MVS-114

MVS-114

MVS-114

$$
\begin{array}{rr}
\text { A } & \text { MVS-114-A } \\
\text { B } & \text { MVS-114-B } \\
\text { C } & \text { MVS-114-C } \\
& \text { mean } \\
\text { stdev } \\
\text { \% RSD }
\end{array}
$$

Sample ID

MVS-121

MVS-121

MVS-121

Sample ID

MVS-130

MVS-130

MVS-130

Sample ID

MVS-139

MVS-139

MVS-139

$$
\begin{array}{cc}
\text { A } & \text { MVS-139-A } \\
\text { B } & \text { MVS-139-B } \\
\text { C } & \text { MVS-139-C } \\
& \text { mean } \\
& \text { stdev }
\end{array}
$$$$
\text { \%RSD }
$$

Sample ID MVS-144 MVS-144 MVS-144

\begin{tabular}{|cc|cc|cc|cc|cc|cc|}
\hline $\mathrm{Fe}$ & $\mathrm{Q}$ & $\mathrm{Cu}$ & $\mathrm{Q}$ & $\mathrm{Pb}$ & $\mathrm{Q}$ & $\mathrm{Zn}$ & $\mathrm{Q}$ & $\mathrm{Cd}$ & $\mathrm{Q}$ & $\mathrm{Ag}$ & $\mathrm{Q}$ \\
2.290 & & 87 & & 50 & & 112 & & 0.0 & $\mathrm{U}$ & 0.4 & $\mathrm{U}$ \\
2.310 & & 87 & & 47 & & 119 & & 0.0 & $\mathrm{U}$ & 1.6 & $\mathrm{~J}$ \\
2.325 & & 80 & & 42 & & 107 & & 0.0 & $\mathrm{U}$ & 0.7 & $\mathrm{U}$ \\
2.308 & & 85 & & 46 & & 113 & 0.0 & $\mathrm{U}$ & 0.9 & $\mathrm{U}$ \\
0.018 & & 4 & & 4 & & 6 & 0.0 & & 0.6 & \\
$\mathbf{1}$ & $\mathbf{5}$ & & $\mathbf{9}$ & & $\mathbf{5}$ & $\mathbf{N A}$ & & $\mathbf{6 6}$ & \\
\hline
\end{tabular}

\begin{tabular}{|cc|cc|cc|cc|cc|cc|}
\hline $\mathrm{Fe}$ & $\mathrm{Q}$ & $\mathrm{Cu}$ & $\mathrm{Q}$ & $\mathrm{Pb}$ & $\mathrm{Q}$ & $\mathrm{Zn}$ & $\mathrm{Q}$ & $\mathrm{Cd}$ & $\mathrm{Q}$ & $\mathrm{Ag}$ & $\mathrm{Q}$ \\
2.436 & & 95 & & 66 & & 132 & & 0.0 & $\mathrm{U}$ & 0.5 & $\mathrm{U}$ \\
2.457 & & 130 & & 63 & & 136 & & 0.0 & $\mathrm{U}$ & 1.1 & $\mathrm{~J}$ \\
2.448 & & 99 & & 68 & & 147 & & 0.1 & $\mathrm{U}$ & 2.2 & $\mathrm{~J}$ \\
2.447 & & 108 & & 66 & & 138 & & 0.0 & $\mathrm{U}$ & 1.3 & $\mathrm{~J}$ \\
0.011 & & 19 & & 2 & & 8 & & 0.1 & & 0.9 & \\
$\mathbf{0}$ & & $\mathbf{1 8}$ & & $\mathbf{4}$ & & $\mathbf{6}$ & $\mathbf{1 7 3}$ & & $\mathbf{6 8}$ & \\
\hline
\end{tabular}

\begin{tabular}{|cc|cc|cc|cc|cc|cc|}
\hline $\mathrm{Fe}$ & $\mathrm{Q}$ & $\mathrm{Cu}$ & $\mathrm{Q}$ & $\mathrm{Pb}$ & $\mathrm{Q}$ & $\mathrm{Zn}$ & $\mathrm{Q}$ & $\mathrm{Cd}$ & $\mathrm{Q}$ & $\mathrm{Ag}$ & $\mathrm{Q}$ \\
2.105 & & 117 & & 41 & & 132 & & 1.9 & $\mathrm{~J}$ & 1.7 & $\mathrm{~J}$ \\
2.110 & & 114 & & 45 & & 133 & & 0.0 & $\mathrm{U}$ & 0.0 & $\mathrm{U}$ \\
2.161 & & 136 & & 42 & & 136 & & 0.0 & $\mathrm{U}$ & 0.2 & $\mathrm{U}$ \\
2.125 & & 122 & & 42 & & 134 & & 0.6 & $\mathrm{U}$ & 0.6 & $\mathrm{U}$ \\
0.031 & & 12 & & 2 & & 2 & & 1.1 & & 0.9 & \\
$\mathbf{1}$ & & $\mathbf{1 0}$ & & $\mathbf{5}$ & & $\mathbf{1}$ & $\mathbf{1 7 3}$ & & $\mathbf{1 4 5}$ & \\
\hline
\end{tabular}

\begin{tabular}{|cc|cc|cc|cc|cc|cc|}
\hline $\mathrm{Fe}$ & $\mathrm{Q}$ & $\mathrm{Cu}$ & $\mathrm{Q}$ & $\mathrm{Pb}$ & $\mathrm{Q}$ & $\mathrm{Zn}$ & $\mathrm{Q}$ & $\mathrm{Cd}$ & $\mathrm{Q}$ & $\mathrm{Ag}$ & $\mathrm{Q}$ \\
2.334 & & 80 & & 55 & & 100 & & 2.4 & $\mathrm{~J}$ & 0.9 & $\mathrm{U}$ \\
2.310 & & 74 & & 45 & & 100 & & 1.4 & $\mathrm{~J}$ & 0.3 & $\mathrm{U}$ \\
2.368 & & 82 & & 46 & & 107 & & 0.6 & $\mathrm{U}$ & 1.4 & $\mathrm{~J}$ \\
2.337 & & 78 & & 49 & & 102 & & 1.5 & $\mathrm{~J}$ & 0.9 & $\mathrm{U}$ \\
0.029 & & 4 & & 5 & & 4 & & 0.9 & & 0.5 & \\
$\mathbf{1}$ & & $\mathbf{5}$ & & $\mathbf{1 1}$ & & $\mathbf{4}$ & $\mathbf{6 1}$ & & $\mathbf{6 1}$ & \\
\hline
\end{tabular}

$$
\begin{array}{rr}
\text { A } & \text { MVS-144-A } \\
\text { B } & \text { MVS-144-B } \\
\text { C } & \text { MVS-144-C } \\
& \text { mean } \\
\text { stdev } \\
\text { \% RSD }
\end{array}
$$

\begin{tabular}{|cc|cc|cc|cc|cc|cc|}
\hline $\mathrm{Fe}$ & $\mathrm{Q}$ & $\mathrm{Cu}$ & $\mathrm{Q}$ & $\mathrm{Pb}$ & $\mathrm{Q}$ & $\mathrm{Zn}$ & $\mathrm{Q}$ & $\mathrm{Cd}$ & $\mathrm{Q}$ & $\mathrm{Ag}$ & $\mathrm{Q}$ \\
2.348 & & 92 & & 50 & & 114 & & 0.6 & $\mathrm{U}$ & 0.8 & $\mathrm{U}$ \\
2.370 & & 85 & & 51 & & 118 & & 1.0 & $\mathrm{~J}$ & 1.5 & $\mathrm{~J}$ \\
2.360 & & 88 & & 54 & & 110 & & 1.2 & $\mathrm{~J}$ & 1.2 & $\mathrm{~J}$ \\
2.359 & & 88 & & 51 & & 114 & & 0.9 & $\mathrm{U}$ & 1.2 & $\mathrm{~J}$ \\
0.011 & & 4 & & 2 & & 4 & & 0.3 & & 0.4 & \\
$\mathbf{0}$ & & $\mathbf{4}$ & & $\mathbf{4}$ & & $\mathbf{3}$ & $\mathbf{3 1}$ & & $\mathbf{3 2}$ & \\
\hline
\end{tabular}


Project: $\quad$ ENVVEST Metals Verification Study (Project No. 43043)

Samples Received: 11/20/2003 (Sinclair Inlet OU B Marine monitoring composites)

Metals Reported: $\quad$ Fe, $\mathrm{Cu}, \mathrm{Pb}, \mathrm{Zn}, \mathrm{Cd}, \mathrm{Ag}$

\section{Data Quality Criteria}

SAMPLE TRIPLICATE (cont'd)

Sample ID

MVS-149

MVS-149

MVS-149

$\begin{array}{rr}\text { A } & \text { MVS-149-A } \\ \text { B } & \text { MVS-149-B } \\ \text { C } & \text { MVS-149-C } \\ & \text { mean } \\ \text { stdev } \\ \text { \% RSD }\end{array}$

\begin{tabular}{|c|c|}
\hline Fe & $\mathrm{Q}$ \\
2.053 & \\
2.090 & \\
2.058 & \\
2.067 & \\
0.020 & \\
$\mathbf{1}$ & \\
\hline
\end{tabular}

\begin{tabular}{cc|c}
$\mathrm{Cu}$ & $\mathrm{Q}$ & $\mathrm{P}$ \\
69 & & 40 \\
71 & \\
75 & & 47 \\
71 & & 37 \\
3 & & 4 \\
4 & & 5 \\
\hline
\end{tabular}

\begin{tabular}{cc|c}
$\mathrm{Pb}$ & $\mathrm{Q}$ & $\mathrm{Zn}$ \\
40 & & 102 \\
47 & & 11 \\
37 & & 95 \\
41 & & 104 \\
5 & & 10 \\
$\mathbf{1 3}$ & & $\mathbf{1 0}$ \\
\hline
\end{tabular}

\begin{tabular}{|cc|cc|cc}
\hline Zn & Q & Cd & Q & Ag & Q \\
102 & & 2.1 & J & 0.1 & U \\
115 & & 1.3 & J & 0.2 & U \\
95 & 0.0 & U & 0.7 & U \\
104 & 1.1 & J & 0.3 & U \\
10 & 1.1 & & 0.3 & \\
$\mathbf{1 0}$ & $\mathbf{9 3}$ & & $\mathbf{9 5}$ & \\
\hline
\end{tabular}

\section{Data Qualifiers (Q):}

$\mathrm{U}$ : The value was less than MDL or the analyte was not detected.

J: Estimated value ( MDL < measured value < LOQ ) 
APPENDIX B

\section{INDUCTIVELY-COUPLED PLASMA-MASS SPECTROSCOPY} DATA REPORT 

PROJECT:

PARAMETER:

LABORATORY:

MATRIX:

SAMPLE CUSTODY AND PROCESSING:
MVS Sediment Confirmatory Analyses

Metals

Battelle Marine Sciences Laboratory, Sequim, Washington

Surface sediments

One hundred and sixty-eight sediment samples were received in two batches at MSL.

The samples were archived frozen until a subset of forty samples for confirmatory metals analyses was selected and analyzed at MSL. All samples were assigned a Battelle Central File (CF) identification number (2087) and were entered into Battelle’s log-in and custody tracking system.

The following lists information on sample receipt and processing activities:

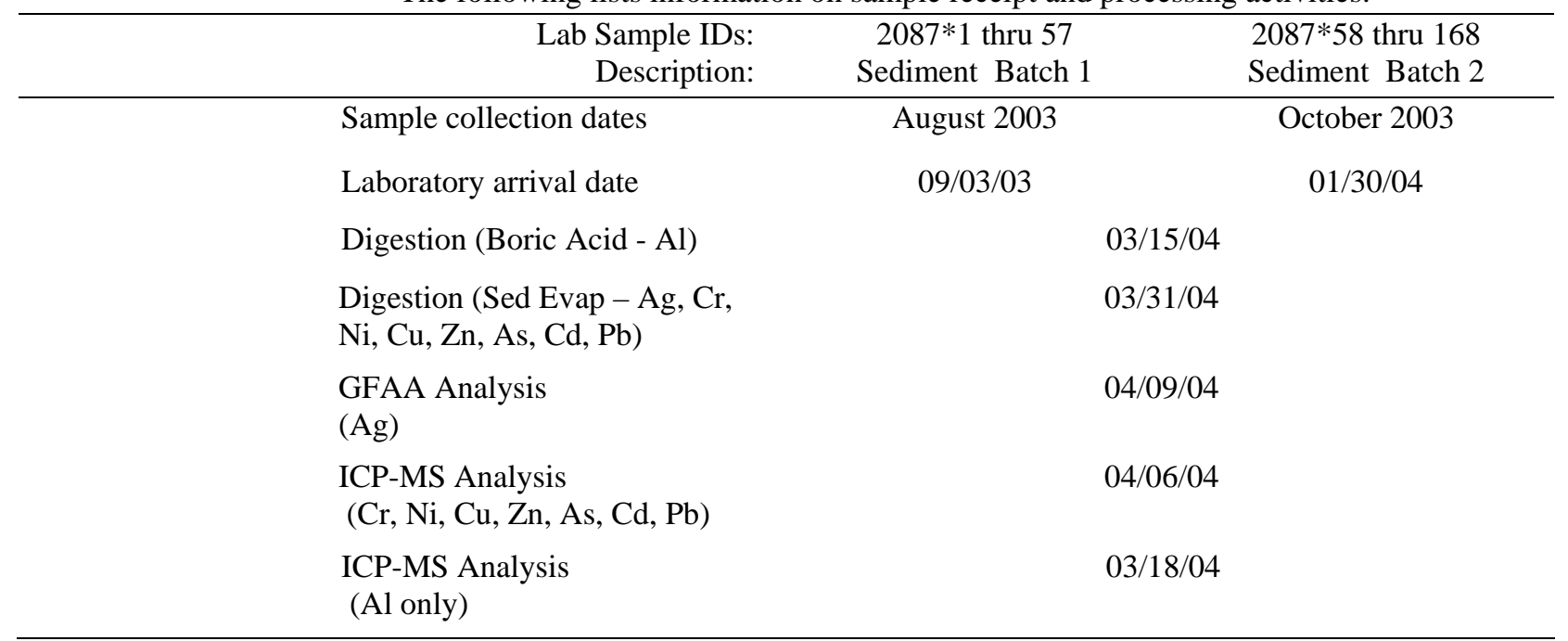

\section{DATA QUALITY OBJECTIVES:}

\begin{tabular}{|c|c|c|c|c|c|c|}
\hline Analyte & $\begin{array}{c}\text { Analytical } \\
\text { Method }\end{array}$ & $\begin{array}{l}\text { Spike Range } \\
\text { of Recovery }\end{array}$ & SRM Accuracy & $\begin{array}{c}\text { Relative } \\
\text { Precision }\end{array}$ & $\begin{array}{c}\text { Achieved } \\
\text { Detection } \\
\text { Limit } \\
\text { (1) }^{\mu g / g ~ d r y ~ w t .)}\end{array}$ & $\begin{array}{c}\text { Reporting } \\
\text { Limit }^{(2)} \\
{\text { ( } \mu \mathrm{g} / \mathrm{g} \text { dry wt.) }}\end{array}$ \\
\hline $\mathrm{Ag}$ & GFAA & $70-130 \%$ & $\leq 20 \%$ PD & $\leq 30 \% \mathrm{RPD}$ & 0.00579 & 0.0184 \\
\hline $\mathrm{Al}$ & ICP-MS & $70-130 \%$ & $\leq 20 \%$ PD & $\leq 30 \%$ RPD & 14.3 & 45.5 \\
\hline As & ICP-MS & $70-130 \%$ & $\leq 20 \%$ PD & $\leq 30 \%$ RPD & 0.188 & 0.598 \\
\hline $\mathrm{Cd}$ & ICP-MS & $70-130 \%$ & $\leq 20 \%$ PD & $\leq 30 \%$ RPD & 0.0587 & 0.187 \\
\hline $\mathrm{Cr}$ & ICP-MS & $70-130 \%$ & $\leq 20 \%$ PD & $\leq 30 \%$ RPD & 0.07 & 0.3 \\
\hline $\mathrm{Cu}$ & ICP-MS & $70-130 \%$ & $\leq 20 \%$ PD & $\leq 30 \%$ RPD & 0.13 & 0.41 \\
\hline $\mathrm{Ni}$ & ICP-MS & $70-130 \%$ & $\leq 20 \%$ PD & $\leq 30 \%$ RPD & 0.0491 & 0.156 \\
\hline $\mathrm{Pb}$ & ICP-MS & $70-130 \%$ & $\leq 20 \%$ PD & $\leq 30 \%$ RPD & 0.0348 & 0.111 \\
\hline $\mathrm{Zn}$ & ICP-MS & $70-130 \%$ & $\leq 20 \%$ PD & $\leq 30 \%$ RPD & 1.02 & 3.24 \\
\hline
\end{tabular}

(1) Reported from the 2003 Sediment MDL summary, Al MDL is corrected for 100x dilution.

(2) Determined as 3.18 times achieved MDL. 
METHODS:

\section{HOLDING} TIMES:

\section{DETECTION LIMITS:}

Sediment samples were analyzed for silver (Ag), aluminum (Al), arsenic (As), cadmium $(\mathrm{Cd})$, chromium $(\mathrm{Cr})$, copper $(\mathrm{Cu})$, lead $(\mathrm{Pb})$, nickel $(\mathrm{Ni})$ and zinc $(\mathrm{Zn})$. Samples were freeze-dried and homogenized using a ball-mill prior to digestion according to Battelle SOP MSL-C-003, Percent Dry Weight and Homogenizing Dry Sediment, Soil and Tissue. Sediment samples were digested using two procedures.

Digestion 1: Boric Acid in accordance with Battelle SOP MSL-I-006, Mixed Acid Sediment Digestion. An approximately 200-mg (dry weight) aliquot of each sample was combined with nitric, hydrochloric, and hydrofluoric acids in a Teflon bomb and heated in an oven at $130^{\circ} \mathrm{C}\left( \pm 10^{\circ} \mathrm{C}\right)$ for a minimum of eight hours. After cooling, boric acid was added to the digestate to neutralize the hydrofluoric acid and deionized water was added to achieve analysis volume. Digested samples were submitted for Al analysis by ICP-MS.

Digestion 2: Sed Evap according to Battelle SOP MSL-I-004, Sediment Evaporation Digestion. An approximately 200-mg (dry weight) aliquot of each sample was combined with nitric acid, hydrofluoric acids and peroxide in a Teflon vessel and heated in an oven at $130^{\circ} \mathrm{C}\left( \pm 10^{\circ} \mathrm{C}\right)$ for a minimum of 8 hours. The digested samples were taken to dryness and reconstituted with dilute nitric acid. Digestates were submitted for analysis by ICP-MS and GFAA (Ag).

Sed Evap sample digestates were analyzed for Ag using graphite furnace atomic absorption (GFAA) according to Battelle SOP MSL-I-029, Determination of Metals in Aqueous and Digestate Samples by GFAA.

Boric Acid sample digestates were analyzed for $\mathrm{Al}$ and Sed Evap samples were analyzed for $\mathrm{As}, \mathrm{Cd}, \mathrm{Cr}, \mathrm{Cu}, \mathrm{Ni}, \mathrm{Pb}$, and $\mathrm{Zn}$ using inductively coupled plasma-mass spectrometry (ICPMS) according to Battelle SOP MSL-I-022, Determination of Elements in Aqueous and Digestate Samples by ICP/MS. All results were reported in units of $\mu \mathrm{g} / \mathrm{g}$ on a dry-weight basis.

The recommended holding time for the reported analytes in sediment is 6 months from sample collection. This holding time was exceeded as screening results were used to select samples for confirmatory analyses. The samples were stored frozen and freeze dried, which is an accepted preservation method. Therefore, exceeding this holding time did not significantly impact the quality of the sample data.

Analytical results were reported to laboratory achieved detection limits derived from the 2003 MDL study. Data were evaluated and flagged in accordance to the following criteria:

U Analyte not detected at or above laboratory achieved detection limit, MDL reported

J Value reported is below the RL, but above the MDL.

\& Accuracy results outside QC criteria of $\leq 20 \%$ PD (SRM).

* Precision results outside QC criteria of $\leq 30 \%$ RPD.

N Spiked sample recovery not within the QC limits of 70-130\%.

B Analyte detected in the method blank above the RL, sample concentration $<10$ times detected blank value.

D Data reported from the second analysis with a dilution of 100x (all other samples are diluted 10x). 
METHOD

BLANKS:

LABORATORY CONTROL SAMPLE ACCURACY:

STANDARD REFERENCE MATERIAL ACCURACY:

MATRIX SPIKE ACCURACY:

LABORATORY PRECISION:
Two method blanks were analyzed with the sediment samples. The average blank was less than the RL for all metals, except Al. The Al data were not flagged "B" because the sample concentrations were all greater than 10 times the average method blank. Data are not blank corrected.

Two laboratory control samples were analyzed with the samples. LCS recoveries ranged from $90 \%$ to $115 \%$ and were within the QC acceptance criterion of $\pm 30 \%$ recovery, with the exception of one LCS sample for $\mathrm{Al}(840 \%)$. No corrective action was taken as the low level spike from this batch and the alternate high level spike were within the QC criterion.

Two marine sediment reference materials for trace metals (SRMs) were analyzed with the sediment samples: MESS-3 and PACS-2. SRM accuracy is expressed as the percent difference (PD) between the certified and measured concentrations.

Two replicates of MESS-3 were analyzed with the samples. The percent differences ranged from $0 \%$ to $12 \%$ and were within the QC acceptance criteria of PD $\pm 20 \%$, with one exception. One replicate for $\mathrm{Al}(21 \%)$ was outside the QC criteria. No corrective action was taken as the alternate batch specific, SRM (PACS), was within QC criterion.

Two replicates of PACS-2 were analyzed with the samples. The percent differences ranged from $2 \%$ to $20 \%$ and were within the QC acceptance criterion for all metals.

Two sediment samples were selected and spiked in duplicate. Matrix spike recoveries ranged from $77 \%$ to $122 \%$ and were within the QC acceptance criterion of $70-130 \%$ recovery for all metals. Since the crustal abundance of $\mathrm{Al}$ is $5-8 \%(\sim 60,000 \mathrm{ppm})$, the samples were not spiked for Al. Analytical accuracy was evaluated by LCS and SRM samples.

Laboratory precision was evaluated using two sets of duplicates and is expressed as the relative percent difference (RPD) of replicate results. The RPD values ranged from $1 \%$ to $8 \%$ and were within the QC criterion of $\leq 30 \%$ RPD. 


\section{BATTELLE MARINE SCIENCES LABORATORIES}

Jill Brandenberger, Project Manager

1529 West Sequim Bay Rd.

Sequim, Washington 98382

(360) 681-4564

\begin{tabular}{|c|c|c|c|c|c|c|c|c|c|c|c|c|c|}
\hline MSL Code & $\begin{array}{l}\text { Station or OUB } \\
\text { Grid Cell }\end{array}$ & Sponsor Code & $\begin{array}{l}\text { Collection } \\
\text { Date }\end{array}$ & $\begin{array}{l}\text { Percent } \\
\text { Moisture }\end{array}$ & Ag & Al & $\mathrm{Cr}$ & $\mathbf{N i}$ & $\mathrm{Cu}$ & Zn & As & Cd & $\mathbf{P b}$ \\
\hline & & & & $\begin{array}{l}\text { Analysis } \\
\text { Batch ID }\end{array}$ & T040904A & $\begin{array}{c}\text { 031804- } \\
6100 A\end{array}$ & $\begin{array}{c}040604- \\
6100\end{array}$ & $\begin{array}{c}040604- \\
6100\end{array}$ & $\begin{array}{c}040604- \\
6100\end{array}$ & $\begin{array}{c}040604- \\
6100\end{array}$ & $\begin{array}{c}040604- \\
6100\end{array}$ & $\begin{array}{c}040604- \\
6100\end{array}$ & $\begin{array}{c}040604- \\
6100\end{array}$ \\
\hline & & & & CAS No. & $7440-22-4$ & 7429-90-5 & $7440-47-3$ & $7440-02-0$ & $7440-50-8$ & $7440-66-6$ & $7440-38-2$ & 7440-43-9 & 7439-92-1 \\
\hline $2087-3$ & MVS-019 & MVS-019-ARC & 08/28/03 & 55.9 & 0.156 & 51700 & 75.7 & 31.6 & 25.7 & 66.1 & 6.38 & 0.631 & 17.6 \\
\hline 2087-10r1 & MVS-024 & MVS-024-ARC & 08/28/03 & 63.0 & 0.484 & 56900 & 95.9 & 41.2 & 54.6 & 113 & 10.4 & 0.986 & 41.7 \\
\hline 2087-10r2 & MVS-024 & MVS-024-ARC & 08/28/03 & 63.0 & 0.448 & 56600 & 95.1 & 41.5 & 52.7 & 116 & 10.5 & 0.966 & 41.2 \\
\hline 2087-11 & MVS-025 & MVS-025-ARC & 08/28/03 & 69.5 & 0.544 & 55100 & 91.1 & 43.1 & 58.7 & 118 & 11.2 & 1.17 & 43.6 \\
\hline $2087-12$ & MVS-026 & MVS-026-ARC & 08/28/03 & 61.1 & 0.489 & 57200 & 100 & 41.6 & 52.7 & 112 & 10.6 & 1.15 & 39.4 \\
\hline 2087-18 & MVS-002 & MVS-002-ARC & 08/29/03 & 28.0 & 0.120 & 52400 & 60.0 & 22.8 & 16.4 & 46.6 & 4.17 & 0.783 & 12.2 \\
\hline \multirow{2}{*}{$\begin{array}{l}2087-20 \\
2087-25\end{array}$} & MVS-007 & MVS-007-ARC & 08/29/03 & 70.7 & 0.790 & 55900 & 96.8 & 49.0 & 78.8 & 159 & 14.8 & 1.26 & 63.8 \\
\hline & MVS-013 & MVS-013-ARC & 08/29/03 & 65.3 & 0.609 & 55400 & 98.7 & 45.6 & 63.0 & 140 & 12.3 & 1.07 & 52.9 \\
\hline 2087-31 & MVS-022 & MVS-022-ARC & 08/29/03 & 62.6 & 0.681 & 57200 & 113 & 45.9 & 65.6 & 132 & 11.8 & 1.80 & 45.4 \\
\hline 2087-32 & MVS-027 & MVS-027-ARC & 08/29/03 & 64.9 & 0.611 & 55100 & 102 & 44.2 & 62.3 & 126 & 10.9 & 1.53 & 44.0 \\
\hline 2087-33 & MVS-028 & MVS-028-ARC & 08/29/03 & 27.8 & 0.127 & 48700 & 67.4 & 30.5 & 17.6 & 50.9 & 5.60 & 0.525 & 12.2 \\
\hline $2087-36$ & MVS-047 & MVS-047-ARC & 08/30/03 & 67.6 & 0.534 & 59700 & 81.3 & 43.7 & 58.8 & 126 & 13.4 & 0.846 & 41.6 \\
\hline $2087-40$ & MVS-056 & MVS-056-ARC & 08/30/03 & 29.5 & 0.0798 & 58800 & 46.7 & 15.7 & 11.8 & 43.4 & 4.29 & 0.656 & 8.69 \\
\hline $2087-43$ & MVS-039 & MVS-039-ARC & 08/30/03 & 19.7 & 0.0559 & 53800 & 43.4 & 27.3 & 11.8 & 47.9 & 4.04 & 0.136 & 11.1 \\
\hline 2087-45 & MVS-043 & MVS-043-ARC & 08/30/03 & 25.5 & 0.0556 & 53800 & 52.1 & 19.1 & 9.56 & 37.9 & 3.83 & 0.184 & 11.1 \\
\hline $2087-52$ & MVS-034 & MVS-034-ARC & 08/31/03 & 25.2 & 0.0635 & 54300 & 71.7 & 24.3 & 10.4 & 41.1 & 3.64 & 0.206 & 9.50 \\
\hline $2087-63$ & OOUB-G 24 & MVS-065-ARC & $10 / 28 / 03$ & 67.2 & 0.705 & 59100 & 87.4 & 45.4 & 107 & 162 & 13.8 & 0.797 & 72.0 \\
\hline $2087-70$ & OUBM-G 52 & MVS-072-ARC & $10 / 24 / 03$ & 62.7 & 1.07 & 57000 & 119 & 79.1 & $398 \mathrm{D}$ & $785 \mathrm{D}$ & 36.1 & 1.30 & 279 \\
\hline $2087-73$ & OOUB-G 01 & MVS-075-ARC & $10 / 30 / 03$ & 29.8 & 0.175 & 50300 & 114 & 97.0 & 102 & $547 \mathrm{D}$ & 15.3 & 0.479 & 198 \\
\hline $2087-76$ & OOUB-G 26 & MVS-078-ARC & $10 / 29 / 03$ & 25.9 & 0.0420 & 58500 & 83.6 & 27.0 & 11.7 & 45.4 & 2.67 & 0.193 & 8.53 \\
\hline 2087-83 & OOUB-G 28 & MVS-085-ARC & $10 / 28 / 03$ & 68.1 & 0.500 & 57000 & 84.5 & 42.6 & 76.1 & 141 & 12.3 & 0.778 & 58.4 \\
\hline $2087-85 r 1$ & OOUB-G 18 & MVS-087-ARC & $10 / 30 / 03$ & 69.6 & 0.943 & 59600 & 96.4 & 47.7 & 117 & 159 & 15.9 & 1.04 & 75.1 \\
\hline $2087-85 r 2$ & OOUB-G 18 & MVS-087-ARC & $10 / 30 / 03$ & 69.6 & 0.925 & 56300 & 97.9 & 48.4 & 118 & 164 & 16.0 & 1.03 & 76.1 \\
\hline $2087-86$ & OOUB-G 21 & MVS-088-ARC & $10 / 30 / 03$ & 67.9 & 0.715 & 60800 & 99.7 & 48.6 & 102 & 159 & 15.9 & 1.32 & 71.8 \\
\hline $2087-90$ & OUBM-G 59 & MVS-092-ARC & $10 / 03 / 03$ & 69.5 & 0.712 & 55900 & 102 & 52.9 & 272 & 736 & 23.1 & 3.17 & $439 \mathrm{D}$ \\
\hline
\end{tabular}




\section{BATTELLE MARINE SCIENCES LABORATORIES}

Jill Brandenberger, Project Manager

1529 West Sequim Bay Rd.

Sequim, Washington 98382

(360) 681-4564

\begin{tabular}{|c|c|c|c|c|c|c|c|c|c|c|c|c|c|}
\hline MSL Code & $\begin{array}{c}\text { Station or OUB } \\
\text { Grid Cell } \\
\end{array}$ & Sponsor Code & $\begin{array}{c}\text { Collection } \\
\text { Date }\end{array}$ & $\begin{array}{c}\text { Percent } \\
\text { Moisture } \\
\end{array}$ & $\mathbf{A g}$ & Al & $\mathrm{Cr}$ & $\mathrm{Ni}$ & $\mathrm{Cu}$ & Zn & As & Cd & $\mathbf{P b}$ \\
\hline & & & & $\begin{array}{l}\text { Analysis } \\
\text { Batch ID }\end{array}$ & T040904A & $\begin{array}{c}\text { 031804- } \\
6100 A\end{array}$ & $\begin{array}{c}040604- \\
6100\end{array}$ & $\begin{array}{c}040604- \\
6100\end{array}$ & $\begin{array}{c}040604- \\
6100\end{array}$ & $\begin{array}{c}040604- \\
6100\end{array}$ & $\begin{array}{c}040604- \\
6100\end{array}$ & $\begin{array}{c}040604- \\
6100\end{array}$ & $\begin{array}{c}040604- \\
6100\end{array}$ \\
\hline & & & & CAS No. & $7440-22-4$ & 7429-90-5 & $7440-47-3$ & $7440-02-0$ & $7440-50-8$ & $7440-66-6$ & $7440-38-2$ & $7440-43-9$ & 7439-92-1 \\
\hline
\end{tabular}

\begin{tabular}{|c|c|c|c|c|c|c|c|c|c|c|c|c|c|}
\hline 2087-92 & OUBM-G 34 & MVS-094-ARC & $10 / 23 / 03$ & 54.7 & 2.38 & 53000 & 91.8 & 45.4 & 171 & 391 & 20.2 & 1.51 & 132 \\
\hline 2087-96 & OUBM-G 40 & MVS-098-ARC & $10 / 22 / 03$ & 13.9 & 0.709 & 58500 & 108 & 49.9 & 135 & 208 & 12.1 & 1.33 & 84.9 \\
\hline 2087-100 & OUBM-G 42 & MVS-102-ARC & $10 / 22 / 03$ & 65.0 & 0.688 & 61000 & 103 & 77.2 & 180 & 188 & 13.1 & 1.06 & 77.8 \\
\hline 2087-105 & OOUB-G 07 & MVS-107-ARC & $10 / 30 / 03$ & 71.1 & 1.35 & 58600 & 109 & 53.4 & 130 & 177 & 17.2 & 2.47 & 72.3 \\
\hline 2087-111 & OUBM-G 46 & MVS-113-ARC & $10 / 23 / 03$ & 40.4 & 0.495 & 52000 & 105 & 40.1 & 142 & $428 \mathrm{D}$ & 15.8 & 0.621 & 155 \\
\hline $2087-113$ & OUBM-G 43 & MVS-115-ARC & $10 / 23 / 03$ & 65.5 & 0.588 & 56700 & 84.0 & 51.4 & 155 & 241 & 15.8 & 1.12 & 74.3 \\
\hline 2087-114 & OOUB-G 27 & MVS-116-ARC & $10 / 28 / 03$ & 68.2 & 0.417 & 59300 & 84.8 & 42.5 & 72.5 & 128 & 11.7 & 1.04 & 55.4 \\
\hline \multirow{2}{*}{$\begin{array}{l}2087-115 \\
2087-130\end{array}$} & OUBM-G 36 & MVS-117-ARC & $10 / 23 / 03$ & 68.7 & 0.838 & 61300 & 99.6 & 59.5 & 150 & 185 & 14.9 & 1.09 & 86.3 \\
\hline & OUBM-G 68 & MVS-132-ARC & $10 / 27 / 03$ & 68.6 & 0.762 & 56600 & 103 & 54.7 & 217 & $526 \mathrm{D}$ & 20.6 & 1.72 & 144 \\
\hline 2087-132 & OUBM-G 56 & MVS-134-ARC & $10 / 03 / 03$ & 71.0 & 0.691 & 59900 & 99.9 & 47.5 & 194 & $324 \mathrm{D}$ & 19.6 & 1.54 & 105 \\
\hline 2087-133 & OUBM-G 66 & MVS-135-ARC & $10 / 02 / 03$ & 66.8 & 0.547 & 55400 & 101 & 46.1 & 227 & $428 \mathrm{D}$ & 15.2 & 1.71 & 159 \\
\hline 2087-139 & OUBM-G 64 & MVS-141-ARC & $10 / 02 / 03$ & 69.7 & 0.777 & 57800 & 98.1 & 51.7 & 230 & $425 \mathrm{D}$ & 19.9 & 1.85 & 209 \\
\hline $2087-140$ & OUBM-G 60 & MVS-142-ARC & $10 / 03 / 03$ & 64.1 & 0.642 & 46800 & 85.7 & 45.5 & 200 & $1480 \mathrm{D}$ & 19.7 & 2.01 & 180 \\
\hline 2087-143 & OUBM-G 67 & MVS-145-ARC & $10 / 03 / 03$ & 62.2 & 1.03 & 56700 & 104 & 479 & $710 \mathrm{D}$ & $547 \mathrm{D}$ & 43.0 & 1.53 & 204 \\
\hline 2087-149 & OUBM-G 39 & MVS-151-ARC & $10 / 23 / 03$ & 45.3 & 1.11 & 51000 & 84.1 & 40.6 & 173 & 288 & 11.9 & 1.39 & 128 \\
\hline $2087-157$ & OUBM-G 45 & MVS-159-ARC & $10 / 22 / 03$ & 60.3 & 0.740 & 55400 & 88.7 & 48.4 & 154 & 238 & 13.1 & 1.44 & 90.0 \\
\hline 2087-167 & OOUB-G 13 & MVS-169-ARC & $10 / 30 / 03$ & 72.5 & 0.971 & 60800 & 98.0 & 48.0 & 116 & 164 & 14.6 & 1.30 & 83.8 \\
\hline
\end{tabular}




\section{BATTELLE MARINE SCIENCES LABORATORIES}

Jill Brandenberger, Project Manager

1529 West Sequim Bay Rd.

Sequim, Washington 98382

(360) 681-4564

\begin{tabular}{|c|c|c|c|c|c|c|c|c|c|c|c|c|c|}
\hline MSL Code & $\begin{array}{l}\text { Station or OUB } \\
\text { Grid Cell }\end{array}$ & Sponsor Code & $\begin{array}{l}\text { Collection } \\
\text { Date }\end{array}$ & $\begin{array}{c}\text { Percent } \\
\text { Moisture }\end{array}$ & Ag & Al & $\mathrm{Cr}$ & $\mathrm{Ni}$ & $\mathrm{Cu}$ & Zn & As & Cd & $\mathbf{P b}$ \\
\hline & & & & $\begin{array}{l}\text { Analysis } \\
\text { Batch ID }\end{array}$ & T040904A & $\begin{array}{c}\text { 031804- } \\
6100 A\end{array}$ & $\begin{array}{c}040604- \\
6100\end{array}$ & $\begin{array}{c}040604- \\
6100\end{array}$ & $\begin{array}{c}040604- \\
6100\end{array}$ & $\begin{array}{c}040604- \\
6100\end{array}$ & $\begin{array}{c}040604- \\
6100\end{array}$ & $\begin{array}{c}040604- \\
6100\end{array}$ & $\begin{array}{c}040604- \\
6100\end{array}$ \\
\hline & & & & CAS No. & $7440-22-4$ & $7429-90-5$ & $7440-47-3$ & $7440-02-0$ & $7440-50-8$ & $7440-66-6$ & $7440-38-2$ & $7440-43-9$ & 7439-92-1 \\
\hline
\end{tabular}

\section{Detection Limits}

Laboratory Achieved Detection Limits

Reporting Limit (3.18* MDL)

\begin{tabular}{|c|c|c|c|c|c|c|c|c|}
\hline & & & & & & \multicolumn{3}{|c|}{0.058} \\
\hline 0.00579 & $14.3^{1}$ & 0.07 & 0.0491 & 0.13 & 1.02 & 0.188 & 7 & 0.0348 \\
\hline 0.0184 & 45.5 & 0.3 & 0.156 & 0.41 & 3.24 & 0.598 & 0.187 & 0.111 \\
\hline $0.0144 \mathrm{~J}$ & 105 & 0.242 & $0.0491 \mathrm{U}$ & $0.13 \mathrm{U}$ & $1.02 \mathrm{U}$ & $0.188 \mathrm{U}$ & $\begin{array}{c}0.058 \\
7 \mathrm{U} \\
0.058\end{array}$ & $0.0348 \mathrm{U}$ \\
\hline $0.0111 \mathrm{~J}$ & 65.8 & $0.200 \mathrm{~J}$ & $0.0491 \mathrm{U}$ & $0.296 \mathrm{~J}$ & $1.02 \mathrm{U}$ & $0.188 \mathrm{U}$ & $7 \mathrm{U}$ & $0.0348 \mathrm{U}$ \\
\hline $0.0128 \mathrm{~J}$ & 85.4 & $0.221 \mathrm{~J}$ & $0.0491 \mathrm{U}$ & $0.148 \mathrm{~J}$ & $1.02 \mathrm{U}$ & $0.188 \mathrm{U}$ & $\begin{array}{l}0.058 \\
7 \mathrm{U}\end{array}$ & $0.0348 \mathrm{U}$ \\
\hline
\end{tabular}

Laboratory Control Sample Results (Blank Spike)

LCS R1 LCS

LCS R2

Mean Blank

Spike Concentration

Percent Recovery, R1

Percent Recovery, R2

LCS

MB

\begin{tabular}{|c|c|c|c|c|c|c|c|c|}
\hline 2.04 & 185 & 2.02 & 2.05 & 2.04 & 2.08 & 2.03 & 2.00 & 2.12 \\
\hline 2.02 & 925 & 2.08 & 2.11 & 2.13 & 2.29 & 2.09 & 2.04 & 2.09 \\
\hline $.0128 \mathrm{~J}$ & 85.4 & $0.221 \mathrm{~J}$ & $0.0491 \mathrm{U}$ & $0.148 \mathrm{~J}$ & $1.02 \mathrm{U}$ & $0.188 \mathrm{U}$ & $\begin{array}{r}0.058 \\
7 \mathrm{U}\end{array}$ & $0.0348 \mathrm{U}$ \\
\hline 2.0 & 100 & 2.0 & 2.0 & 2.0 & 2.0 & 2.0 & $\begin{array}{r}2.0 \\
100\end{array}$ & 2.0 \\
\hline $101 \%$ & $100 \%$ & $90 \%$ & $103 \%$ & $95 \%$ & $104 \%$ & $102 \%$ & $\begin{array}{r}\% \\
102\end{array}$ & $106 \%$ \\
\hline $1 \%$ & $840 \% \mathrm{~N}$ & $93 \%$ & $106 \%$ & $99 \%$ & $115 \%$ & $105 \%$ & $\%$ & $105 \%$ \\
\hline
\end{tabular}




\section{BATTELLE MARINE SCIENCES LABORATORIES}

Jill Brandenberger, Project Manager

1529 West Sequim Bay Rd.

Sequim, Washington 98382

(360) 681-4564

\begin{tabular}{|c|c|c|c|c|c|c|c|c|c|c|c|c|c|}
\hline MSL Code & $\begin{array}{c}\text { Station or OUB } \\
\text { Grid Cell }\end{array}$ & Sponsor Code & $\begin{array}{c}\text { Collection } \\
\text { Date }\end{array}$ & $\begin{array}{l}\text { Percent } \\
\text { Moisture } \\
\end{array}$ & Ag & Al & $\mathrm{Cr}$ & $\mathbf{N i}$ & $\mathrm{Cu}$ & Zn & As & Cd & $\mathbf{P b}$ \\
\hline & & & & $\begin{array}{l}\text { Analysis } \\
\text { Batch ID }\end{array}$ & T040904A & $\begin{array}{c}031804- \\
6100 A\end{array}$ & $\begin{array}{c}040604- \\
6100\end{array}$ & $\begin{array}{c}040604- \\
6100\end{array}$ & $\begin{array}{c}040604- \\
6100\end{array}$ & $\begin{array}{c}040604- \\
6100\end{array}$ & $\begin{array}{c}040604- \\
6100\end{array}$ & $\begin{array}{c}040604- \\
6100\end{array}$ & $\begin{array}{c}040604- \\
6100\end{array}$ \\
\hline & & & & CAS No. & $7440-22-4$ & $7429-90-5$ & $7440-47-3$ & $7440-02-0$ & $7440-50-8$ & $7440-66-6$ & $7440-38-2$ & $7440-43-9$ & 7439-92-1 \\
\hline
\end{tabular}

\section{Standard Reference Material (SRM)}

MESS-3 R1 SRM

MESS-3 R2

SRM

Certified Value

$\underset{v}{\varpi}$

Range

Range
PD

$\mathrm{PD}$

PACS R1

PACS R2

SRM

Certified Value

Range

PD

$\mathrm{PD}$

$\begin{array}{rrrrrrrrr}0.165 & 77700 & 103 & 44.7 & 36.3 & 149 & 22.2 & 0.217 & 21.3 \\ 0.170 & 68100 & 100 & 41.9 & 33.9 & 142 & 21.8 & 0.211 & 21.1 \\ 0.18 & 85900 & 105 & 46.9 & 33.9 & 159 & 21.2 & 0.24 & 21.1 \\ \pm 0.02 & \pm 2300 & \pm 4 & \pm 2.2 & \pm 1.6 & \pm 8 & \pm 1.1 & 0.01 & \pm 0.7 \\ \mathbf{8 \%} & \mathbf{1 0 \%} & \mathbf{2 \%} & \mathbf{5 \%} & \mathbf{7 \%} & \mathbf{6 \%} & \mathbf{5 \%} & \mathbf{1 0 \%} & \mathbf{1 \%} \\ \mathbf{5 \%} & \mathbf{2 1 \%} \& & \mathbf{5 \%} & \mathbf{1 1 \%} & \mathbf{0 \%} & \mathbf{1 1 \%} & \mathbf{3 \%} & \mathbf{1 2 \%} & \mathbf{0 \%} \\ & & & & & & & & \\ 0.993 & 62500 & 81.8 & 36.5 & 275 & 340 & 27.4 & 2.07 & 173 \\ 0.978 & 57800 & 85.4 & 38.8 & 291 & 356 & 29.0 & 2.07 & 173 \\ & & & & & & & & \\ 1.22 & 66200 & 90.7 & 39.5 & 310 & 364 & 26.2 & 2.11 & 183 \\ \pm 0.14 & \pm 3200 & \pm 4.6 & \pm 2.3 & \pm 12 & \pm 23 & \pm 1.5 & \pm 0.2 & \pm 8 \\ \mathbf{1 9 \%} & \mathbf{6 \%} & \mathbf{1 0 \%} & \mathbf{8 \%} & \mathbf{1 1 \%} & \mathbf{7 \%} & \mathbf{5 \%} & \mathbf{2 \%} & \mathbf{5 \%} \\ \mathbf{2 0} \% & \mathbf{1 3 \%} & \mathbf{6 \%} & \mathbf{2 \%} & \mathbf{6 \%} & \mathbf{2 \%} & \mathbf{1 1 \%} & \mathbf{2 \%} & \mathbf{5 \%}\end{array}$




\section{BATTELLE MARINE SCIENCES LABORATORIES}

Jill Brandenberger, Project Manager

1529 West Sequim Bay Rd.

Sequim, Washington 98382

(360) 681-4564

\begin{tabular}{|c|c|c|c|c|c|c|c|c|c|c|c|c|c|}
\hline MSL Code & $\begin{array}{l}\text { Station or OUB } \\
\text { Grid Cell }\end{array}$ & Sponsor Code & $\begin{array}{l}\text { Collection } \\
\text { Date }\end{array}$ & $\begin{array}{c}\text { Percent } \\
\text { Moisture }\end{array}$ & Ag & Al & $\mathrm{Cr}$ & Ni & $\mathrm{Cu}$ & Zn & As & Cd & $\mathbf{P b}$ \\
\hline & & & & $\begin{array}{l}\text { Analysis } \\
\text { Batch ID }\end{array}$ & T040904A & $\begin{array}{c}031804- \\
6100 A\end{array}$ & $\begin{array}{c}040604- \\
6100\end{array}$ & $\begin{array}{c}040604- \\
6100\end{array}$ & $\begin{array}{c}040604- \\
6100\end{array}$ & $\begin{array}{c}040604- \\
6100\end{array}$ & $\begin{array}{c}040604- \\
6100\end{array}$ & $\begin{array}{c}040604- \\
6100\end{array}$ & $\begin{array}{c}040604- \\
6100\end{array}$ \\
\hline & & & & CAS No. & $7440-22-4$ & $7429-90-5$ & $7440-47-3$ & $7440-02-0$ & $7440-50-8$ & $7440-66-6$ & $7440-38-2$ & $7440-43-9$ & $7439-92-1$ \\
\hline
\end{tabular}

\section{Matrix Spike/Matrix Spike Duplicate Results}

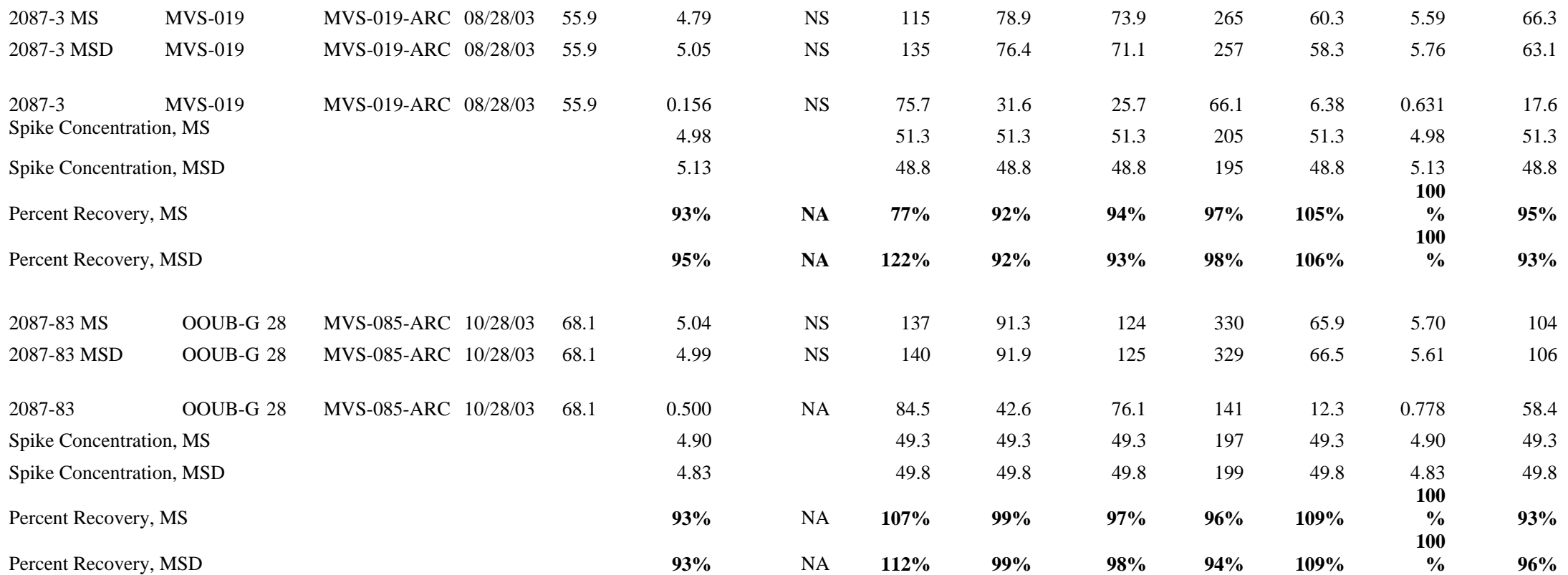

Percent Recovery, MSD 


\title{
BATTELLE MARINE SCIENCES LABORATORIES
}

Jill Brandenberger, Project Manager

1529 West Sequim Bay Rd.

Sequim, Washington 98382

(360) 681-4564

\begin{tabular}{|c|c|c|c|c|c|c|c|c|c|c|c|c|c|}
\hline MSL Code & $\begin{array}{c}\text { Station or OUB } \\
\text { Grid Cell }\end{array}$ & Sponsor Code & $\begin{array}{c}\text { Collection } \\
\text { Date }\end{array}$ & $\begin{array}{c}\text { Percent } \\
\text { Moisture } \\
\end{array}$ & Ag & Al & $\mathrm{Cr}$ & $\mathrm{Ni}$ & $\mathrm{Cu}$ & $\mathrm{Zn}$ & As & Cd & $\mathbf{P b}$ \\
\hline & & & & $\begin{array}{l}\text { Analysis } \\
\text { Batch ID }\end{array}$ & T040904A & $\begin{array}{c}031804- \\
6100 A\end{array}$ & $\begin{array}{c}040604- \\
6100\end{array}$ & $\begin{array}{c}040604- \\
6100\end{array}$ & $\begin{array}{c}040604- \\
6100\end{array}$ & $\begin{array}{c}040604- \\
6100\end{array}$ & $\begin{array}{c}040604- \\
6100\end{array}$ & $\begin{array}{c}040604- \\
6100\end{array}$ & $\begin{array}{c}040604- \\
6100\end{array}$ \\
\hline & & & & CAS No. & $7440-22-4$ & 7429-90-5 & $7440-47-3$ & $7440-02-0$ & $7440-50-8$ & $7440-66-6$ & $7440-38-2$ & $7440-43-9$ & 7439-92-1 \\
\hline
\end{tabular}

\section{Replicate Analysis Results}

2087-10r1 MVS-024

2087-10r2 MVS-024

MVS-024-ARC $\quad 08 / 28 / 03 \quad 63.0$

MEAN

MVS-024-ARC 08/28/03 63.0

0.448

0.466

95.9

41.2

1.5

54.6

113

10.4

RPD

\author{
2087-85r2 \\ OOUB-G 18 \\ MVS-087-ARC $\quad 10 / 30 / 03 \quad 69.6$ \\ MEAN \\ OOUB-G 18 MVS-087-ARC 10/30/03 69.6 \\ RPD \\ ${ }^{1}$ Sed Evap MDL, adjusted for 100x dilution \\ D Reported from dilution analysis (100x) \\ U Concentration less than the MDL reported \\ J Estimated concentration > MDL, but less than the RL \\ B Blank $>$ RL and sample concentration $<10$ times detected blank. \\ NA Not applicable/available \\ NS Not spiked \\ N Spiked sample recovery outside QC criterion of $\pm 30 \%$ \\ \& Accuracy outside QC criterion of $\pm 20 \%$ difference (SRMs) \\ * Precision outside QC criterion of $\leq 30 \%$ RPD
}

$8 \%$

56750

95.5

41.4

53.7

115

10.5

0.986

0.966

41.7

0.943

$0.943 \quad 59600$

$1 \%$

$4 \%$

$3 \%$

41.2

$\begin{array}{rrr}0.934 & 57950 & 97.2 \\ \mathbf{2 \%} & \mathbf{6 \%} & \mathbf{2 \%}\end{array}$

\section{7}

117

159

164

$1 \%$

2\%

41.5 